\title{
SPACE OF RICCI FLOWS (II)-PART A: MODULI OF SINGULAR CALABI-YAU SPACES
}

\author{
XIUXIONG CHEN $^{1,2}$ and BING WANG ${ }^{3}$ \\ ${ }^{1}$ Department of Mathematics, Stony Brook University, Stony Brook, NY 11794, USA \\ ${ }^{2}$ School of Mathematics, University of Science and Technology of China, Hefei, \\ Anhui 230026, PR China; \\ email: xiu@math.sunysb.edu \\ ${ }^{3}$ Department of Mathematics, University of Wisconsin-Madison, Madison, WI 53706, USA; \\ email: bwang@math.wisc.edu
}

Received 31 October 2016; accepted 24 November 2017

\begin{abstract}
We establish the compactness of the moduli space of noncollapsed Calabi-Yau spaces with mild singularities. Based on this compactness result, we develop a new approach to study the weak compactness of Riemannian manifolds.
\end{abstract}

2010 Mathematics Subject Classification: 53C25 (primary); 53C23 (secondary)

\section{Introduction}

Motivated by the celebrated work [34] of Perelman, we are interested in studying the model spaces of Kähler-Ricci flow solutions with bounded scalar curvature. It is not hard to observe that such model spaces must be Ricci-flat spaces, possibly with mild singularities. In Chen and Wang [15], we study Kähler-Ricci flow on Fano surfaces and the model spaces are nothing but Kähler-Ricci-flat orbifolds with nonzero asymptotic volume ratio. However, in higher dimension, the choice of the model spaces becomes much more complicated. The pointed-GromovHausdorff limits of smooth Calabi-Yau manifolds with asymptotic volume ratio bounded below by $\kappa>0$ are plausible model spaces. If we select the fundamental properties of these limit spaces and collect all singular Calabi-Yau manifolds which satisfy this set of key properties, it turns out that is the correct candidate for 
the moduli of model spaces. We denote it by $\widetilde{\mathscr{K} \mathscr{S}}(n, \kappa)$ and its precise definition can be found in Definition 2.1.

THEOREM 1.1 (Structure of model moduli). $\widetilde{\mathscr{K} \mathscr{S}}(n, \kappa)$ is compact under the pointed- $\hat{C}^{\infty}$-Cheeger-Gromov topology. In other words, for each sequence of $\left(X_{i}, x_{i}, g_{i}\right) \in \widehat{\mathscr{K} \mathscr{S}}(n, \kappa)$, by taking subsequence if necessary, we have

$$
\left(X_{i}, x_{i}, g_{i}\right) \stackrel{\hat{C}^{\infty}}{\longrightarrow}(\bar{X}, \bar{x}, \bar{g})
$$

for some $(\bar{X}, \bar{x}, \bar{g}) \in \widetilde{\mathscr{K} \mathscr{S}}(n, \kappa)$. Moreover, each space $X \in \widetilde{\mathscr{K} \mathscr{S}}(n, \kappa)$ is a Calabi-Yau conifold.

Note that the convergence topology in (1.1) was stated as 'pointed-CheegerGromov' topology previously in the literature, for example, in Chen and Wang [15]. We now use extra term $\hat{C}^{\infty}$ to emphasize that it deals with singularities. Note that 'Cheeger-Gromov' means 'modulo diffeomorphisms' as usual. Let us say a few more words about its precise meaning. In fact, (1.1) first means that $\left(X_{i}, x_{i}, d_{i}\right)$ in Gromov-Hausdorff topology converges to a pointed-length-space $(\bar{X}, \bar{x}, \bar{d})$, where $d_{i}$ is the length structure induced by $g_{i}$. The second meaning of (1.1) is that $\bar{X}$ has a regular-singular decomposition $\bar{X}=\mathcal{R}(\bar{X}) \cup \mathcal{S}(\bar{X})$, where the regular part $\mathcal{R}(\bar{X})$ is a smooth manifold equipped with a smooth metric $\bar{g}$, the singular part $\mathcal{S}(\bar{X})$ is a measure (2n-dimensional Hausdorff measure) zero set. Locally, around each regular point, the metric structure determined by $\bar{g}$ is identical to $\bar{d}$. The regular part $\mathcal{R}(\bar{X})$ has an exhaustion $\bigcup_{j=1}^{\infty} K_{j}$ by compact sets $K_{j}$. For any compact subset $K$, one can find diffeomorphisms $\varphi_{K, i}$ from $K$ to $\varphi_{K, i}(K)$, a subset of $\mathcal{R}\left(X_{i}\right)$ such that

$$
\begin{gathered}
d_{i}\left(\varphi_{K, i}(y), x_{i}\right) \rightarrow d_{\infty}(y, \bar{x}), \quad \forall y \in K ; \\
\varphi_{K, i}^{*}\left(g_{i}\right) \stackrel{C^{\infty}}{\longrightarrow} \bar{g} \text { on } K .
\end{gathered}
$$

Although in general the global distance structure induced by $\bar{g}$ may not be the same as $\bar{d}$, this difference does not happen whenever the limit space $\bar{X} \in \widetilde{\mathscr{K} \mathscr{S}}(n, \kappa)$ since $\mathcal{R}(\bar{X})$ is weakly geodesically convex. Clearly, $\infty$ can be replaced by general positive $k$ and the convergence in the pointed- $\hat{C}^{k}$-CheegerGromov topology can be defined similarly.

The notion of conifold is well known to string theorist as some special CalabiYau 3-folds with singularities (see [26]). In this paper, by abusing notation, we use it to denote a space whose singular part admits cone type tangent spaces. The precise definition of conifold is given as follows. 
Definition 1.2. A length space $\left(X^{n}, g\right)$ is called a conifold of complex dimension $n$ if the following properties are satisfied.

(1) $X$ has a disjoint regular-singular decomposition $X=\mathcal{R} \cup \mathcal{S}$, where $\mathcal{R}$ is the regular part, $\mathcal{S}$ is the singular part. A point is called regular if it has a neighborhood which is isometric to a geodesically convex domain in some smooth Riemannian manifold. A point is called singular if it is not regular.

(2) The regular part $\mathcal{R}$ is a nonempty, open manifold of real dimension $2 n$. Moreover, there exists a complex structure $J$ on $\mathcal{R}$ such that $(\mathcal{R}, g, J)$ is a Kähler manifold.

(3) $\mathcal{R}$ is strongly convex, that is, for every two points $x \in \mathcal{R}$ and $y \in X$, one can find a shortest geodesic $\gamma$ connecting $x, y$ where every interior point is in $\mathcal{R}$. In particular, $\mathcal{R}$ is geodesically convex.

(4) $\operatorname{dim}_{\mathcal{M}} \mathcal{S} \leqslant 2 n-4$, where $\operatorname{dim}_{\mathcal{M}}$ means Minkowski dimension (see Definition 2.2).

(5) Every tangent space of $x \in \mathcal{S}$ is a metric cone of Hausdorff dimension $2 n$. Moreover, if $Y$ is a tangent cone of $x$, then the unit ball $B(\hat{x}, 1)$ centered at vertex $\hat{x}$ must satisfy

$$
|B(\hat{x}, 1)|_{d \mu} \leqslant\left(1-\delta_{0}\right) \omega_{2 n},
$$

for some uniform positive number $\delta_{0}=\delta_{0}(n)$. Here $d \mu$ is the $2 n$-dimensional Hausdorff measure, $\omega_{2 n}$ is the volume of the unit ball in $\mathbb{C}^{n}$.

A Calabi-Yau conifold is a conifold which is Ricci-flat on the regular part. Note that Calabi-Yau conifold is a generalization of Calabi-Yau orbifold. Furthermore, one can also define Riemannian conifold of real dimension $m$ verbatim. Most results of this paper apply also to Ricci-flat Riemannian conifolds. The proofs also follow verbatim from those in the current paper, except for the high codimension argument of singularity (see Proposition 2.55). For more details about the motivation and history of the definition of conifold, see the discussion before Conjecture 2.59 at the end of Section 2.6.

Theorem 1.1 consists of two parts. The first part is the compactness of the moduli space $\widetilde{\mathscr{K} \mathscr{S}}(n, \kappa)$. The second part is the improving regularity of spaces in $\widehat{\mathscr{K} \mathscr{S}}(n, \kappa)$. Theorem 1.1 is motivated by section 11 of Perelman's seminal paper [34], where Perelman proved the compactness of moduli spaces of $\kappa$-solutions and showed that $\kappa$-solutions have many properties which are not obvious from the definition. The first part of Theorem 1.1 can be stated as follows. 
THEOREM 1.3 (Compactness). $\widetilde{\mathscr{K} \mathscr{S}}(n, \kappa)$ is compact under the pointed- $\hat{C}^{\infty}$ Cheeger-Gromov topology. In particular, $\widetilde{\mathscr{K} \mathscr{S}}(n, \kappa)$ is compact under the pointed-Gromov-Hausdorff topology.

The strategy to prove the compactness of $\widetilde{\mathscr{K} \mathscr{S}}(n, \kappa)$ follows the same route of the weak-compactness theory of Kähler-Einstein manifolds, developed by Cheeger, Gromoll, Anderson, Colding, Tian, Naber, and so on. However, the analysis foundations, like integration by parts, maximum principle on the singular spaces need to be carefully checked. This is discussed in Section 2 of this paper. After we check the analysis foundation, we then follow the routine routes to develop the weak compactness (see [18] for more details) of $\widetilde{\mathscr{K} \mathscr{S}}(n, \kappa)$. In other words, for a sequence of $\left(X_{i}, x_{i}, g_{i}\right) \in \widetilde{\mathscr{K} \mathscr{S}}(n, \kappa)$, we have (1.1) holds without knowing that $(\bar{X}, \bar{x}, \bar{g}) \in \widetilde{\mathscr{K} \mathscr{S}}(n, \kappa)$. However, as $\mathscr{\mathscr { K } \mathscr { S }}(n, \kappa)$ consists of spaces with mild singularities, one can further show that the limit space $(\bar{X}, \bar{x}, \bar{g})$ must also lie in $\widehat{\mathscr{K} \mathscr{S}}(n, \kappa)$. Therefore, we obtain the compactness of $\overline{\mathscr{K} \mathscr{S}}(n, \kappa)$, rather than the weak compactness.

Besides the compactness, the moduli $\widetilde{\mathscr{K} \mathscr{S}}(n, \kappa)$ has another advantage: it is straightforward to check whether $X$ is in $\widetilde{\mathscr{K} \mathscr{S}}(n, \kappa)$ or not by verifying the 6 defining properties (see Definition 2.1). There are other compact moduli spaces of singular spaces, for example, $\overline{\mathscr{K} \mathscr{S}}(n, \kappa)$, the closure of noncollapsed CalabiYau manifolds under the pointed-Gromov-Hausdorff topology. However, it is a hard problem to check whether a given space $X$ locates in $\overline{\mathscr{K} \mathscr{S}}(n, \kappa)$. In other words, it is difficult to check whether a singular space $X$ can be approximated by a sequence of smooth Calabi-Yau manifolds.

The second part of Theorem 1.1 claims that each space $X \in \widetilde{\mathscr{K} \mathscr{S}}(n, \kappa)$ has better regularity than what is prescribed by its definition. We explain it in more details as the following theorem.

THEOREM 1.4 (Space regularity improvement). Suppose $X \in \widetilde{\mathscr{K} \mathscr{S}}(n, \kappa)$, then $\mathcal{R}$ is strongly convex, and $\operatorname{dim}_{\mathcal{M}} \mathcal{S} \leqslant 2 n-4$. Suppose $x_{0} \in \mathcal{S}$ and $Y$ is a tangent space of $X$ at $x_{0}$. Then $Y$ is a metric cone in $\widetilde{\mathscr{K} \mathscr{S}}(n, \kappa)$ with the splitting

$$
Y=\mathbb{C}^{n-k} \times C(Z)
$$

for some $k \geqslant 2$, where $C(Z)$ is a metric cone without lines.

Here $\operatorname{dim}_{\mathcal{M}}$ means the Minkowski dimension (see Definition 2.2). By strong convexity, we mean that for every two points $x \in \mathcal{R}$ and $y \in X$, one can 
find a shortest geodesic $\gamma$ connecting $x, y$ where every interior point is in $\mathcal{R}$. Theorem 1.4 is basically an application of Theorem 1.3.

The compactness theorem, Theorem 1.3 can be understood in terms of $a$ priori estimates of many geometric quantities.

THEOREM 1.5 (A priori estimates in model spaces). Suppose $\left(X, x_{0}, g\right) \in$ $\widetilde{\mathscr{K} \mathscr{S}}(n, \kappa), r$ is a positive number. Then the following estimates hold.

(1) Strong volume ratio estimate: $\kappa \leqslant \omega_{2 n}^{-1} r^{-2 n}\left|B\left(x_{0}, r\right)\right| \leqslant 1$.

(2) Strong regularity estimate: $r^{2+k}\left|\nabla^{k} R m\right| \leqslant c_{a}^{-2}$ in the ball $B\left(x_{0}, c_{a} r\right)$ for every $0 \leqslant k \leqslant 5$ whenever $\mathbf{v r}\left(x_{0}\right) \geqslant r$.

(3) Strong density estimate: $r^{2 p_{0}-2 n} \int_{B\left(x_{0}, r\right)} \operatorname{vr}(y)^{-2 p_{0}} d y \leqslant \mathbf{E}$.

(4) Strong connectivity estimate: Every two points $y_{1}, y_{2} \subset B\left(x_{0}, r\right) \cap$ $\mathcal{F}_{(1 / 100)_{c_{b} r}}(X)$ can be connected by a shortest geodesic $\gamma$ such that $\gamma \subset \mathcal{F}_{\epsilon_{b} r}(X)$.

Let us explain some of the notations in Theorem 1.5. The constants $c_{a}, c_{b}, \epsilon_{b}$ all depend on $\kappa$ and $n$, the constant $p_{0}$ depends only on $n$ and it is very close to 2 , say $p_{0}=2-(1 / 1000 n)$, the constant $\mathbf{E}$ depends on $\kappa, n$ and $p_{0}$. More precise definitions and motivations of them can be found in the beginning of section 3.1. The function vr is the volume radius, whose precise definition is given in Definition 2.45. For each $r>0, \mathcal{F}_{r}(X)$ means the part of $X$ where volume radius is at least $r$. For its precise meaning, see Definition 2.53. The number ' 5 ' in the strong regularity estimate can be replaced by any number big enough.

Motivated by Theorem 1.5, we can define a scale as the maximum radius such that all the rough versions of estimates in Theorem 1.5 hold under that radius. Such scale is called the canonical radius with respect to the model space $\widetilde{\mathscr{K} \mathscr{S}}(n, \kappa)$ (see Definition 3.5 for the precise meaning). We denote the canonical radius by cr. Under the assumption of cr being uniformly bounded from below, one can develop a weak-compactness theory of manifolds. Here we use the term 'weak' since the limit space in general has worse regularity than the spaces before taking the limit.

THEOREM 1.6 (Rough weak compactness). Suppose $\left(M_{i}, g_{i}, J_{i}\right)$ is a sequence of complete Kähler manifolds satisfying $\mathbf{c r}\left(M_{i}\right) \geqslant r_{0}>0$ uniformly. Let $d_{i}$ be the length structure induced by $g_{i}$. By taking subsequence if necessary, we have

$$
\left(M_{i}, x_{i}, d_{i}\right) \stackrel{\text { P.G.H. }}{\longrightarrow}(\bar{M}, \bar{x}, \bar{d}) .
$$


Then we have the regular-singular decomposition $\bar{M}=\mathcal{R} \cup \mathcal{S}$ with the following properties.

- The regular part $\mathcal{R}$ is an open, path connected $C^{4}$-Riemannian manifold. Furthermore, for every two points $x, y \in \mathcal{R}$, there exists a curve $\gamma$ connecting $x$, $y$ satisfying

$$
\gamma \subset \mathcal{R}, \quad|\gamma| \leqslant 3 d(x, y) .
$$

- The singular part $\mathcal{S}$ satisfies the Minkowski dimension estimate

$$
\operatorname{dim}_{\mathcal{M}} \mathcal{S} \leqslant 2 n-2 p_{0} .
$$

Furthermore, the convergence of (1.2) can be improved as

$$
\left(M_{i}, x_{i}, g_{i}\right) \stackrel{\hat{C}^{4}}{\rightarrow}(\bar{M}, \bar{x}, \bar{g}) .
$$

Let us explain in a few more words about the meaning of (1.5). It means (1.2) together with the extra information that the convergence on $\mathcal{R}(\bar{M})$ happens in the $C^{4}$-topology modulo diffeomorphisms. It is important to note that the length structure of $\bar{d}$ is not necessarily equivalent to the length structure induced by $\bar{g}$. Instead, only a rough equivalence (1.3) is known. Of course, in most interesting cases, we do want the two length structures to coincide. However, it is an important step which need extra information beyond the canonical radius assumption. For example, if $\left(M_{i}, x_{i}, g_{i}\right)$ is a blowup sequence of Kähler-Einstein manifolds with bounded scalar curvature, then one can use the monotonicity of Einstein manifolds to show that $\mathcal{R}(\bar{M})$ is geodesically convex. In particular, the length structure induced by $\bar{g}$ is exactly $\vec{d}$. However, other monotonicities may play a similar role. In a subsequent paper, we shall show that if $\left(M_{i}, x_{i}, g_{i}\right)$ are time slices of noncollapsed Kähler-Ricci flow solutions with $|R| \rightarrow 0$, then the expected coincidence of the two length structures does hold. The monotonicity of the Ricci flows, discovered by Perelman [34], will play an essential role to achieve this goal.

Note that the canonical radius used in Theorem 1.6 is the one defined with respect to the model moduli space $\mathscr{K} \mathscr{S}(n, \kappa)$ (see Definition 3.5). One can define other canonical radii with respect to different model moduli space. For example, if we choose the moduli $\mathscr{M}$ as the collection of all Euclidean spaces of dimension $2 n$, then $\mathscr{M}$ consists of only one element $\left(\mathbb{R}^{2 n}, g\right)$ and has automatic compactness under the smooth topology. In this case, we can regard the classical harmonic radius (see Anderson [2]) as the canonical radius with respect to the moduli $\mathscr{M}$. Further discussion of canonical radius can be found in Remarks 3.20, 3.21, at the end of Section 3.3. 
This paper and a subsequent paper [17] originate from a single paper [16]. In the subsequent paper [17], we shall use Theorems 1.3, 1.5 and 1.6 to show the convergence of the Kähler-Ricci flow on Fano manifolds. In particular, we shall prove the following weak-compactness theorem, together with some applications.

THEOREM 1.7 (Weak compactness of flows, see Chen and Wang [17, Theorem 1.6]). Suppose $\left\{\left(M^{n}, g(t)\right), 0 \leqslant t<\infty\right\}$ is an anticanonical KählerRicci flow solution on a Fano manifold $(M, J)$. For every $s>1$, define

$$
\begin{aligned}
g_{s}(t) & \triangleq g(t+s), \\
\mathcal{M}_{s} & \triangleq\left\{\left(M^{n}, g_{s}(t)\right),-s \leqslant t \leqslant s\right\} .
\end{aligned}
$$

Then for every sequence $s_{i} \rightarrow \infty$, by taking subsequence if necessary, we have

$$
\left(\mathcal{M}_{s_{i}}, g_{s_{i}}\right) \stackrel{\hat{C}^{\infty}}{\longrightarrow}(\overline{\mathcal{M}}, \bar{g})
$$

where the limit space-time $\overline{\mathcal{M}}$ is a Kähler-Ricci soliton flow solution on a $Q$-Fano normal variety $(\bar{M}, \bar{J})$. Moreover, with respect to each $\bar{g}(t)$, there is a uniform $C$ independent of time such that the $r$-neighborhood of the singular set $\mathcal{S}$ has measure not greater than $\mathrm{Cr}^{4}$.

Therefore, the current paper is the technical foundation of the subsequent paper Chen and Wang [17]. However, the main results of this paper, that is, Theorem 1.1 to Theorem 1.6, may have their own interests, although they are motivated by the study of the Kähler-Ricci flow on Fano manifolds.

The organization of this paper is as follows. In Section 2, we prove the compactness theorem, that is, Theorem 1.3 and its equivalent version, Theorem 1.5. As an application of Theorem 1.3, we show the regularity improvement theorem, that is, Theorem 1.4 and the main theorem, Theorem 1.1. In Section 3, we define the canonical radius and develop a weak-compactness theory for manifolds whose canonical radii are uniformly bounded from below.

\section{Model space-Calabi-Yau space with mild singularities}

In this section, we shall discuss the properties of some model space, from the perspective of metric space structure and the intrinsic Ricci flow structure.

2.1. Singular Calabi-Yau space $\widetilde{\mathscr{K} \mathscr{S}}(\boldsymbol{n}, \boldsymbol{\kappa})$. Let $\mathscr{K} \mathscr{S}(n)$ be the class of all the complete $n$-dimensional Calabi-Yau (Kähler-Ricci-flat) manifolds. 
Let $X \in \mathscr{K} \mathscr{S}(n), x \in X$. By Bishop-Gromov comparison, it is clear that the limit

$$
\lim _{r \rightarrow \infty} \frac{|B(x, r)|}{r^{2 n}}
$$

exists and does not depend on the choice of $x$. Namely, the asymptotic volume ratio is well defined for every manifold in the moduli space $\mathscr{K} \mathscr{S}(n)$. The gap theorem of Anderson (see [2, Lemma 3.1]) implies that the asymptotic volume ratio is strictly less than $1-2 \delta_{0}$ whenever the underlying manifold is not the flat $\mathbb{C}^{n}$, where $\delta_{0}$ is a positive dimensional constant. We fix this constant and call it Anderson constant in this paper.

Let $\mathscr{K} \mathscr{S}(n, \kappa)$ be a subspace of $\mathscr{K} \mathscr{S}(n)$ which consists of elements whose asymptotic volume ratio is at least $\kappa$. Clearly, $\mathscr{K} \mathscr{S}(n, \kappa)$ is not compact under the pointed-Gromov-Hausdorff topology. It can be compactified as a space $\overline{\mathscr{K} \mathscr{S}}(n, \kappa)$. However, this may not be the largest space that one can develop weak-compactness theory. So we extend the space $\overline{\mathscr{K} \mathscr{S}}(n, \kappa)$ further to a possibly bigger compact space $\widetilde{\mathscr{K} \mathscr{S}}(n, \kappa)$, which is defined as follows.

Definition 2.1. Let $\widetilde{\mathscr{K} \mathscr{S}}(n, \kappa)$ be the class of length spaces $(X, g)$ with the following properties.

(1) $X$ has a disjoint regular-singular decomposition $X=\mathcal{R} \cup \mathcal{S}$, where $\mathcal{R}$ is the regular part, $\mathcal{S}$ is the singular part. A point is called regular if it has a neighborhood which is isometric to a geodesically convex domain of some smooth Riemannian manifold. A point is called singular if it is not regular.

(2) The regular part $\mathcal{R}$ is a nonempty, open Ricci-flat (with respect to the Riemannian metric $g$ on $\mathcal{R}$ ) manifold of real dimension $m=2 n$. Moreover, there exists a complex structure $J$ on $\mathcal{R}$ such that $(\mathcal{R}, g, J)$ is a Kähler manifold.

(3) $\mathcal{R}$ is weakly convex, that is, for every point $x \in \mathcal{R}$, there exists a measure (2n-dimensional Hausdorff measure) zero set $\mathcal{C}_{x} \supset \mathcal{S}$ such that every point in $X \backslash \mathcal{C}_{x}$ can be connected to $x$ by a unique shortest geodesic in $\mathcal{R}$. For convenience, we call $\mathcal{C}_{x}$ the cut locus of $x$.

(4) $\operatorname{dim}_{\mathcal{M}} \mathcal{S}<2 n-3$, where $\mathcal{M}$ means Minkowski dimension.

(5) Let $\mathrm{v}$ be the volume density function, that is,

$$
\mathrm{v}(x) \triangleq \lim _{r \rightarrow 0} \frac{|B(x, r)|}{\omega_{2 n} r^{2 n}}
$$


for every $x \in X$. Then $\mathrm{v} \equiv 1$ on $\mathcal{R}$ and $\mathrm{v} \leqslant 1-2 \delta_{0}$ on $\mathcal{S}$. In other words, the function $\mathrm{v}$ is a criterion function for singularity. Here $\delta_{0}$ is the Anderson constant.

(6) The asymptotic volume ratio $\operatorname{avr}(X) \geqslant \kappa$. In other words, we have

$$
\lim _{r \rightarrow \infty} \frac{|B(x, r)|}{\omega_{2 n} r^{2 n}} \geqslant \kappa
$$

for every $x \in X$.

Let $\widetilde{\mathscr{K} \mathscr{S}}(n)$ be the class of length spaces $(X, g)$ with all the above properties except the last one. Since Euclidean space is a special element, we define

$$
\widetilde{\mathscr{K} \mathscr{S}}^{*}(n) \triangleq \widetilde{\mathscr{K} \mathscr{S}}(n) \backslash\left\{\left(\mathbb{C}^{n}, g_{\mathbb{E}}\right)\right\}, \quad \widetilde{\mathscr{K} \mathscr{S}}^{*}(n, \kappa) \triangleq \widetilde{\mathscr{K} \mathscr{S}}(n, \kappa) \backslash\left\{\left(\mathbb{C}^{n}, g_{\mathbb{E}}\right)\right\} .
$$

There is an abuse of notation in Definition 2.1. Every space $X \in \widetilde{\mathscr{K} \mathscr{S}}(n, \kappa)$ has a default length structure $d$, which is induced by the smooth Riemannian manifold $(\mathcal{R}, g)$ because of the third and fourth properties in Definition 2.1. We write $(X, g)$ for the simplicity of notation. Note that the $\kappa$ in $\widetilde{\mathscr{K} \mathscr{S}}(n, \kappa)$ means the asymptotic volume ratio is at least $\kappa$. If we drop $\kappa$, the space $\widetilde{\mathscr{K} \mathscr{S}}(n)$ may contain compact spaces. The default measure is always the $2 n$ dimensional Hausdorff measure, unless we mention otherwise. We use $\operatorname{dim}_{\mathcal{H}}$ to denote Hausdorff dimension, $\operatorname{dim}_{\mathcal{M}}$ to denote Minkowski dimension, or the box-counting dimension. Since Minkowski dimension is not as often used as Hausdorff dimension, let us recall the definition of it quickly (see [23]).

Definition 2.2. Suppose $E$ is a bounded subset of $X$, and $E_{r}$ is the $r$-neighborhood of $E$ in $X$. Then the upper Minkowski dimension of $E$ is defined as the limit: $\operatorname{dim}_{\mathcal{H}} X-\liminf \operatorname{in}_{r \rightarrow 0^{+}}\left(\log \left|E_{r}\right| / \log r\right)$. We say $\operatorname{dim}_{\mathcal{M}} E \leqslant \operatorname{dim}_{\mathcal{H}} X-k$ if the upper Minkowski dimension of $E$ is not greater than $2 n-k$. Namely, we have

$$
\liminf _{r \rightarrow 0^{+}} \frac{\log \left|E_{r}\right|}{\log r} \geqslant k
$$

If $E$ is an unbounded set, we say $\operatorname{dim}_{\mathcal{M}} E \leqslant \operatorname{dim}_{\mathcal{H}} X-k$ if $\operatorname{dim}_{\mathcal{M}} E \cap B \leqslant$ $\operatorname{dim}_{\mathcal{H}} X-k$ for each unit geodesic ball $B \subset X$ satisfying $B \cap E \neq \emptyset$.

In general, it is known that Hausdorff dimension is not greater than Minkowski dimension. Hence, we always have $\operatorname{dim}_{\mathcal{H}} \mathcal{S} \leqslant \operatorname{dim}_{\mathcal{M}} \mathcal{S}$. In our discussion, $X$ clearly has Hausdorff dimension $2 n$. Therefore, $\operatorname{dim}_{\mathcal{M}} \mathcal{S}<2 n-3$ implies that for each nonempty intersection $B\left(x_{0}, 1\right) \cap \mathcal{S}$, its $r$-neighborhood has measure $o\left(r^{3}\right)$ 
for sufficiently small $r$. By virtue of the high codimension of $\mathcal{S}$ and the Ricci flatness of $\mathcal{R}$, in many aspects, each metric space $X \in \widetilde{\mathscr{K} \mathscr{S}}(n, \kappa)$ can be treated as an intrinsic Ricci-flat space. We shall see that the geometry of $X$ is almost the same as that of a Calabi-Yau manifold.

Proposition 2.3 (Bishop-Gromov volume comparison). Suppose $x_{0} \in X$, $0<r_{a}<r_{b}<\infty$, and $\delta>0$. Then we have

$$
\begin{gathered}
\frac{\left|B\left(x_{0}, r_{a}\right)\right|}{r_{a}^{2 n}} \geqslant \frac{\left|B\left(x_{0}, r_{b}\right)\right|}{r_{b}^{2 n}}, \\
\frac{\left|B\left(x_{0}, r_{a}+\delta\right)\right|-\left|B\left(x_{0}, r_{a}\right)\right|}{\left(r_{a}+\delta\right)^{2 n}-r_{a}^{2 n}} \geqslant \frac{\left|B\left(x_{0}, r_{b}+\delta\right)\right|-\left|B\left(x_{0}, r_{b}\right)\right|}{\left(r_{b}+\delta\right)^{2 n}-r_{b}^{2 n}} .
\end{gathered}
$$

Proof. We first prove (2.2) for the case $x_{0} \in \mathcal{R}$. Away from the cut locus $\mathcal{C}_{x_{0}}$, which is measure zero, every point can be connected to $x_{0}$ by a unique smooth geodesic. Therefore, every point $y \in X \backslash \mathcal{C}_{x_{0}}$ can be identified with a point $L \gamma^{\prime}(0) \in \mathbb{R}^{2 n}$, where $\gamma$ is a unit-speed shortest geodesic connecting $x_{0}$ and $y$, with $\gamma(0)=x_{0}, L$ is the length of $\gamma$. In this way, we constructed a polar coordinate system around $x_{0}$. Since $\left|B\left(x_{0}, r\right)\right|=\left|B\left(x_{0}, r\right) \backslash \mathcal{C}_{x_{0}}\right|$, by calculating the volume element evolution along each $\gamma$ in polar coordinate, we obtain the volume comparison the same as the Riemannian case. This is more or less standard. For example, one can check the details from [44], or the survey [43]. Now we show (2.2) for $x_{0} \in \mathcal{S}$. Let $x_{i} \in \mathcal{R}$ and $x_{i} \rightarrow x_{0}$. Fix $r>0$. Note that

$$
\lim _{i \rightarrow \infty}\left|B\left(x_{i}, r\right)\right|=|B(x, r)| .
$$

Actually, for each $\epsilon>0$ and large $i$, we have $B\left(x_{i}, r-\epsilon\right) \subset B(x, r) \subset B\left(x_{i}, r+\epsilon\right)$ and hence

$$
\begin{gathered}
\left|B\left(x_{i}, r-\epsilon\right)\right|-|B(x, r)| \leqslant\left|B\left(x_{i}, r\right)\right|-|B(x, r)| \leqslant\left|B\left(x_{i}, r+\epsilon\right)\right|-|B(x, r)|, \\
|| B\left(x_{i}, r\right)|-| B(x, r)|| \leqslant\left|B\left(x_{i}, r+\epsilon\right)-B\left(x_{i}, r-\epsilon\right)\right| .
\end{gathered}
$$

Note that $x_{i}$ is a regular point for each $i>1$, by standard Bishop-Gromov comparison, we have

$$
\left|B\left(x_{i}, r+\epsilon\right)-B\left(x_{i}, r-\epsilon\right)\right| \leqslant 2 n \omega_{2 n}\left\{(r+\epsilon)^{2 n}-(r-\epsilon)^{2 n}\right\} \leqslant C(n, r) \epsilon .
$$

Therefore, taking limit of (2.5) as $i \rightarrow \infty$ and then let $\epsilon \rightarrow 0$, we obtain (2.4). Consequently, we have

$$
\lim _{i \rightarrow \infty} \omega_{2 n}^{-1} r_{a}^{-2 n}\left|B\left(x_{i}, r_{a}\right)\right|=\omega_{2 n}^{-1} r_{a}^{-2 n}\left|B\left(x_{0}, r_{a}\right)\right|,
$$




$$
\lim _{i \rightarrow \infty} \omega_{2 n}^{-1} r_{b}^{-2 n}\left|B\left(x_{i}, r_{b}\right)\right|=\omega_{2 n}^{-1} r_{b}^{-2 n}\left|B\left(x_{0}, r_{b}\right)\right|
$$

Again, $x_{i}$ is a regular point for each $i>1$, so (2.2) was proved for $x_{i}$ and can be written as

$$
\omega_{2 n}^{-1} r_{a}^{-2 n}\left|B\left(x_{i}, r_{a}\right)\right| \geqslant \omega_{2 n}^{-1} r_{b}^{-2 n}\left|B\left(x_{i}, r_{b}\right)\right|
$$

Plugging the above inequality into (2.6), we obtain (2.2) for the singular point $x_{0}$.

The proof of (2.3) is similar. We first prove (2.3) for regular point $x_{0}$ and then use approximation to prove it for singular $x_{0}$. For regular $x_{0}$, in polar coordinates, (2.3) can be proved the same as the smooth Riemannian manifold case (see [44, Theorem 3.1]). In the approximation step, it is important to have volume continuity of annulus. However, this can be proved similar to (2.4), by using triangle inequalities.

COROLlary 2.4 (Volume doubling). $X$ is a volume doubling metric space. More precisely, for every $x_{0} \in X$ and $r>0$, we have

$$
\frac{\left|B\left(x_{0}, 2 r\right)\right|}{\left|B\left(x_{0}, r\right)\right|} \leqslant \kappa^{-1}
$$

COROLlary 2.5 ('Area ratio' monotonicity). For each $x_{0} \in X$, there is $a$ function $A(r)$, the 'area ratio', defined almost everywhere on $(0, \infty)$ such that

$$
\begin{gathered}
\left|B\left(x_{0}, r\right)\right|=\int_{0}^{r} A(s) s^{2 n-1} d s, \quad \forall r>0 . \\
\frac{\left|B\left(x_{0}, r_{b}\right)\right|}{r_{b}^{2 n}}-\frac{\left|B\left(x_{0}, r_{a}\right)\right|}{r_{a}^{2 n}}=\int_{r_{a}}^{r_{b}} \frac{2 n}{r}\left(\frac{A(r)}{2 n}-\frac{\left|B\left(x_{0}, r\right)\right|}{r^{2 n}}\right) d r, \quad \forall 0<r_{a}<r_{b} .
\end{gathered}
$$

Furthermore, $A$ is nonincreasing on its domain. In other words, we have $A\left(r_{a}\right) \geqslant A\left(r_{b}\right)$ whenever $A\left(r_{a}\right), A\left(r_{b}\right)$ are well defined and $0<r_{a}<r_{b}$.

Proof. From the approximation process in the proof of Proposition 2.3, we see that even for $x_{0} \in \mathcal{S}$, the inequalities

$$
0 \leqslant \frac{d}{d r}\left|B\left(x_{0}, r\right)\right| \leqslant 2 n \omega_{2 n} r^{2 n-1}, \quad-\frac{2 n}{r} \leqslant \frac{d}{d r}\left\{\frac{\left|B\left(x_{0}, r\right)\right|}{\omega_{2 n} r^{2 n}}\right\} \leqslant 0,
$$

hold in the barrier sense. In particular, $\left|B\left(x_{0}, r\right)\right|$ and $\omega_{2 n} r^{-2 n}\left|B\left(x_{0}, r\right)\right|$ are monotone, uniformly Lipschitz functions of $r$ on each compact subinterval of $(0, \infty)$. Therefore, they have bounded derivatives almost everywhere. By abuse of notation, we denote the derivatives of $\left|B\left(x_{0}, r\right)\right|$ by $\left|\partial B\left(x_{0}, r\right)\right|$. Denote 
$r^{1-2 n}\left|\partial B\left(x_{0}, r\right)\right|$ by $A(r)$. Clearly, $A(r)$ is defined almost everywhere on $(0, \infty)$. Intuitively, $A(r)$ is the area ratio of geodesic sphere. By absolute continuity of $\left|B\left(x_{0}, r\right)\right|$ and $r^{-2 n}\left|B\left(x_{0}, r\right)\right|,(2.7)$ and (2.8) are nothing but the Newton-Leibniz formula.

We now show the monotonicity of $A$. Actually, suppose $A\left(r_{a}\right)$ and $A\left(r_{b}\right)$ are well defined. Then we have

$$
\begin{aligned}
A\left(r_{a}\right) & =\lim _{\epsilon \rightarrow 0^{+}} \frac{\left|B\left(x_{0}, r_{a}+\epsilon\right)\right|-\left|B\left(x_{0}, r_{a}\right)\right|}{r_{a}^{2 n-1} \epsilon} \\
& =\lim _{\epsilon \rightarrow 0^{+}} \frac{2 n\left\{\left|B\left(x_{0}, r_{a}+\epsilon\right)\right|-\left|B\left(x_{0}, r_{a}\right)\right|\right\}}{\left(r_{a}+\epsilon\right)^{2 n}-r_{a}^{2 n}}, \\
A\left(r_{b}\right) & =\lim _{\epsilon \rightarrow 0^{+}} \frac{\left|B\left(x_{0}, r_{b}+\epsilon\right)\right|-\left|B\left(x_{0}, r_{b}\right)\right|}{r_{b}^{2 n-1} \epsilon} \\
& =\lim _{\epsilon \rightarrow 0^{+}} \frac{2 n\left\{\left|B\left(x_{0}, r_{b}+\epsilon\right)\right|-\left|B\left(x_{0}, r_{b}\right)\right|\right\}}{\left(r_{b}+\epsilon\right)^{2 n}-r_{b}^{2 n}} .
\end{aligned}
$$

Following from (2.3) and the above identities, we obtain $A\left(r_{a}\right) \geqslant A\left(r_{b}\right)$ by taking limits.

Proposition 2.6 (Segment inequality). For every nonnegative function $f \in$ $L_{\mathrm{loc}}^{1}(X)$, define

$$
\mathcal{F}_{f}\left(x_{1}, x_{2}\right) \triangleq \inf _{\gamma} \int_{0}^{l} f(\gamma(s)) d s,
$$

where the infimum is taken over all minimal geodesics $\gamma$, from $x_{1}$ to $x_{2}$ and $s$ denotes the arc length. Suppose $p \in X, r>0, A_{1}, A_{2}$ are two subsets of $B(p, r)$. Then we have

$$
\int_{A_{1} \times A_{2}} \mathcal{F}_{f}\left(x_{1}, x_{2}\right) \leqslant 4^{n} r\left(\left|A_{1}\right|+\left|A_{2}\right|\right) \int_{B(p, 3 r)} f .
$$

Proof. Fix a smooth point $x_{1}$, then away from cut locus, every point can be connected to $x_{1}$ by a unique geodesic. Since $X \times X$ is equipped with the product measure, it is clear that away from a measure-zero set, every point $\left(x_{1}, x_{2}\right) \in X \times X$ has the property that $x_{1}$ and $x_{2}$ are smooth and can be joined by a unique smooth shortest geodesic. Then the proof of (2.9) follows analogous to the Riemannian manifold case. The interested readers can find the details in the work of Cheeger and Colding in [8].

Due to the work of Cheeger and Colding (see [8, Remark 2.82]), the segment inequality implies the $(1,2)$-Poincaré inequality in general. In our particular case, the Poincaré constant can be understood more precisely. 
Proposition 2.7 (Bound of Poincaré constant). Suppose $f \in L_{\mathrm{loc}}^{1}(X), h$ is an upper gradient of $f$ in the sense of Cheeger (see Definition 2.9). Then for every geodesic ball $B(p, r) \subset X$ and real number $q \geqslant 1$, we have

$$
f_{B(p, r)}|f-\underline{f}| \leqslant 2 \cdot 6^{2 n} \cdot r\left(f_{B(p, 3 r)} h^{q}\right)^{1 / q},
$$

where $f$ means the average, $f$ is the average of $f$ on $B(p, r)$. In particular, there is a uniform $(1,2)$-Poincaré constant on $X$.

Proof. This is standard. For example, one can check [8] and references therein for the details.

Proposition 2.8 (Bound of Sobolev constant). There is a uniform isoperimetric constant on $X$. Consequently, a uniform $L^{2}$-Sobolev inequality holds on $X$. Namely, there is a constant $C_{S}=C_{S}(n, \kappa)$ such that

$$
\left\{\int_{X}|f|^{2 m /(m-2)}\right\}^{(m-2) / m} \leqslant C_{S} \int_{X}|\nabla f|^{2}
$$

for every function $f \in N_{c}^{1,2}(X)$ (see Definition 2.10), where $m=2 n$.

Proof. Due to the uniform noncollapsing condition and the weak convexity and Ricci flatness of $\mathcal{R}$, the argument of Croke (see [21]) applies. So there is a uniform isoperimetric constant on $X$. Alternatively, one can use Coulhon and Saloff [20, Theorem 3] to obtain the uniform isoperimetric constant on $X$, since it has uniform Euclidean volume growth rate and uniform Poincaré constant. This means that for each $f \in N_{c}^{1,2}(X)$, we have

$$
\left\{\int_{X}|f|^{m /(m-1)}\right\}^{(m-1) / m} \leqslant C_{I} \int_{X}|\nabla f| .
$$

Replacing $|f|$ by $|f|^{(2(m-1)) /(m-2)}$ in the above inequality, the $L_{2}$-Sobolev inequality then follows from the above inequality and Hölder inequality.

Note that for each $X \in \widetilde{\mathscr{K} \mathscr{S}}(n, \kappa)$, we lose smooth structure around $\mathcal{S}$. In orbifold case, one can recover the smooth structure at a local 'covering' space. For general $X$, there is no smooth structure at all. However, the good news is that the smooth structure does not play an essential role in many aspects. In the next subsection, we shall see that the analysis on $X$ is almost the same as that on a manifold. 
2.2. Sobolev space, Dirichlet form and heat semigroup. On a metric measure space, one can define Sobolev space $H_{1,2}(X)$ following Cheeger [5], or $N^{1,2}(X)$ following Shanmugalingam [35]. However, these two definitions coincide whenever volume doubling property and uniform $(1,2)$-Poincaré inequality holds, in light of [35, Theorem 4.10], or the discussion on page 440 of [5]. In particular, for the space $(X, g, d \mu)$ which we are interested in, we have $N^{1,2}(X)=H_{1,2}(X)$ as Banach spaces. Here $d \mu$ is the $2 n$-dimensional Hausdorff measure. For simplicity, we shall only use the notation $N^{1,2}(X)$ and follow the approach of Cheeger.

Definition 2.9 (See [5, Definition 1.1]). Suppose $\Omega \subset X$. Let $f: \Omega \rightarrow[0, \infty]$ be an extended function. An extended real function $h: \Omega \rightarrow[0, \infty]$ is called an upper gradient of $f$ on $\Omega$ if for every two points $z_{1}, z_{2} \in \Omega$ and all continuous rectifiable curves $c:[0, l] \rightarrow \Omega$, parameterized by arc length $s$, with $z_{1}, z_{2}$ end points, we have

$$
\left|f\left(z_{1}\right)-f\left(z_{2}\right)\right| \leqslant \int_{0}^{l} h(c(s)) d s .
$$

Definition 2.10 (See [5, Definition 2.2]). The Sobolev space $N^{1,2}(X)$ is the subspace of $L^{2}(X)$ consisting of functions $f$ for which the norm

$$
\|f\|_{N^{1,2}}^{2}=\|f\|_{L^{2}}^{2}+\inf _{f_{i}} \liminf _{i \rightarrow \infty}\left\|h_{i}\right\|_{L^{2}}^{2}<\infty
$$

where the limit infimum is taken over all upper gradients $h_{i}$ of the functions $f_{i}$, which satisfies $\left\|f_{i}-f\right\|_{L^{2}(X)} \rightarrow 0$.

Note that the above $N^{1,2}$-norm is equivalent to Cheeger's definition (see [5, Equation (2.1)]). With this norm, we know $N^{1,2}(X)$ is complete (see [5, Theorem 2.7]). Clearly, it follows directly from the definition that zero function $f \in L^{2}(X)$ is the zero function in $N^{1,2}(X)$. It is not surprising that $N^{1,2}(X)$ is the classical Sobolev space when $X$ is a smooth manifold. This can be easily proved following the same argument as in the proof of Theorem 4.5 of [35], where the same conclusion was proved when $X$ is a domain of Euclidean space. In particular, as Banach spaces, we have

$$
N^{1,2}(\mathcal{R}) \cong W^{1,2}(\mathcal{R}),
$$

where $W^{1,2}(\mathcal{R})$ is the classical Sobolev space on the smooth manifold $\mathcal{R}$.

Proposition 2.11 (Smooth approximation). Suppose $\Omega$ is an open set of $X$, $f \in N^{1,2}(\Omega)$. Then there is a sequence of $f_{i} \in C^{\infty}(\Omega \backslash \mathcal{S}) \cap N^{1,2}(\Omega)$, with 
supp $f_{i} \subset \Omega \backslash \mathcal{S}$ such that

$$
\lim _{i \rightarrow \infty}\left\|f_{i}-f\right\|_{N^{1,2}(\Omega)}=0
$$

Moreover, if $f$ is nonnegative, we can choose the approximation $f_{i}$ nonnegative. If $\Omega$ is bounded, then supp $f_{i}$ is a compact subset of $\bar{\Omega} \backslash \mathcal{S}$.

Proof. It suffices to show the proof for the case when both $\operatorname{diam}(\Omega)$ and $\|f\|_{L^{\infty}}$ are bounded, since for the general case we can work with the truncated function, and use a diagonal sequence argument.

Since $\mathcal{S}$ has measure zero, and $\Omega \backslash \mathcal{S}$ is a smooth manifold, we have

$$
\left\|f_{i}-f\right\|_{N^{1,2}(\Omega)}=\left\|f_{i}-f\right\|_{N^{1,2}(\Omega \backslash \mathcal{S})}=\left\|f_{i}-f\right\|_{W^{1,2}(\Omega \backslash \mathcal{S})} .
$$

Therefore, (2.13) is equivalent to

$$
\lim _{i \rightarrow \infty}\left\|f_{i}-f\right\|_{W^{1,2}(\Omega \backslash \mathcal{S})}=0 .
$$

This sequence of $f_{i}$ can be constructed following a standard method, as indicated by the proof of Theorem 2 of Section 5.3.2 of Evans' book [22]. For the convenience of the readers, we include a detailed construction of $f_{i}$ here.

For each positive integer $i$, define

$$
\Omega_{i} \triangleq\left\{y \in \Omega \mid d(y, \mathcal{S})>2^{-i}\right\}, \quad V_{i} \triangleq \Omega_{i+3} \backslash \bar{\Omega}_{i+1}, \quad W_{i} \triangleq \Omega_{i+4} \backslash \bar{\Omega}_{i} .
$$

Also, choose open sets $V_{0}$ and $W_{0}$ such that

$$
\bar{\Omega}_{4} \cap \Omega \supset V_{0} \supset \bar{\Omega}_{2} \cap \Omega, \quad W_{0} \supset \bar{\Omega}_{6} \cap \Omega \supset V_{0} .
$$

Then we have

$$
\Omega \backslash \mathcal{S}=\bigcup_{i=0}^{\infty} V_{i}=\bigcup_{i=0}^{\infty} W_{i}, \quad \bar{V}_{i} \cap \Omega_{i} \subset W_{i}, \quad \forall i \geqslant 0 .
$$

Clearly, we can choose Lipschitz cutoff functions $\zeta_{i}$ that depends only on $d(\cdot, \mathcal{S})$ such that $\zeta_{i}=1$ on $V_{i}$ and $\operatorname{supp} \zeta_{i} \subset W_{i},\left|\nabla \zeta_{i}\right|<2^{i+5}$. Set $\eta_{i} \triangleq \zeta_{i} /\left(\sum_{j} \zeta_{j}\right)$. Clearly, $\eta_{i}$ is a kind of partition of unity subordinate to the covering $\bigcup_{i} W_{i}$. In other words, we have

$$
\begin{cases}0 \leqslant \eta_{i} \leqslant 1 & \eta_{i} \in C_{c}^{1}\left(W_{i}\right), \quad \forall i \geqslant 1, \\ \sum_{i} \eta_{i}=1 & \text { on } \Omega \backslash \mathcal{S} .\end{cases}
$$


Note that $\eta_{0}$ is special. It is only in $C^{1}\left(W_{0}\right)$ in general. However, it vanishes around $\partial W_{0} \cap \Omega$. For each $i \geqslant 0$, note that $V_{i} \cap V_{j}=\emptyset$ if $|i-j| \geqslant 2, W_{i} \cap W_{j}=\emptyset$ if $|i-j| \geqslant 4$. Therefore, we have

$$
0 \leqslant \eta_{i}<1, \quad\left|\nabla \eta_{i}\right|<2^{i+10} .
$$

For each $i \geqslant 1$, we see that $\eta_{i} f \in W_{0}^{1,2}\left(W_{i}\right)$. Note that $W_{i} \subset \mathcal{R}$. Applying convolution with smooth mollifiers (see Theorem 1 of Section 5.3.1 of [22]), we can choose a smooth function $h_{i} \in C_{c}^{\infty}\left(W_{i}\right)$ such that

$$
\left\|h_{i}-\eta_{i} f\right\|_{W^{1,2}(\Omega \backslash \mathcal{S})}^{2}=\left\|h_{i}-\eta_{i} f\right\|_{W^{1,2}\left(W_{i}\right)}^{2}<9^{-i-1} \epsilon^{2} .
$$

For $i=0$, we can choose $h_{0} \in C^{\infty}\left(W_{0}\right)$ which vanishes in a neighborhood of $\partial W_{0} \cap \Omega$ such that the above inequality holds. For each large $k$, we define $H_{k} \triangleq$ $\sum_{i=0}^{k} h_{i}$. Then $H_{k} \in C^{\infty}\left(\bigcup_{i=0}^{k} W_{i}\right) \subset C^{\infty}(\Omega \backslash \mathcal{S})$. Moreover, we have estimate

$$
\begin{aligned}
\left\|H_{k}-f\right\|_{W^{1,2}(\Omega \backslash \mathcal{S})} & =\left\|\sum_{i=0}^{k} h_{i}-\sum_{i=1}^{\infty} \eta_{i} f\right\|_{W^{1,2}(\Omega \backslash \mathcal{S})} \\
& =\left\|\sum_{i=0}^{k}\left(h_{i}-\eta_{i} f\right)-\sum_{i=k+1}^{\infty} \eta_{i} f\right\|_{W^{1,2}(\Omega \backslash \mathcal{S})} \\
& \leqslant \sum_{i=0}^{k}\left\|h_{i}-\eta_{i} f\right\|_{W^{1,2}(\Omega \backslash \mathcal{S})}+\left\|\sum_{i=k+1}^{\infty} \eta_{i} f\right\|_{W^{1,2}(\Omega \backslash \mathcal{S})} .
\end{aligned}
$$

The first term on the right hand side of the above inequality can be bounded as follows.

$$
\sum_{i=0}^{k}\left\|h_{i}-\eta_{i} f\right\|_{W^{1,2}(\Omega \backslash \mathcal{S})}<\sum_{i=0}^{k} 3^{-i-1} \epsilon<\frac{1}{2} \epsilon .
$$

On the other hand, note that $\sum_{i=k+1}^{\infty} \eta_{i}=1$ on $\bigcup_{i=k+5}^{\infty} W_{i}$, and it is supported on $\bigcup_{i=k+1}^{\infty} W_{i}$. Thus, we have

$$
\begin{aligned}
\left\|\sum_{i=k+1}^{\infty} \eta_{i} f\right\|_{W^{1,2}(\Omega \backslash \mathcal{S})}^{2} & \leqslant\left\|\sum_{i=k+1}^{\infty} \eta_{i} f\right\|_{W^{1,2}\left(\bigcup_{i=k+5}^{\infty} W_{i}\right)}^{2}+\left\|\sum_{i=k+1}^{\infty} \eta_{i} f\right\|_{W^{1,2}\left(\bigcup_{i=k+1}^{k+4} W_{i}\right)}^{2} \\
& =\|f\|_{W^{1,2}\left(\bigcup_{i=k+5}^{\infty} W_{i}\right)}^{2}+\left\|\sum_{i=k+1}^{k+8} \eta_{i} f\right\|_{W^{1,2}\left(\bigcup_{i=k+1}^{k+4} W_{i}\right)}^{2}
\end{aligned}
$$


For simplicity of notation, define $\chi_{k} \triangleq \sum_{i=k+1}^{k+8} \eta_{i}$. Clearly, $0 \leqslant \chi_{k} \leqslant 1$. We have

$$
\begin{aligned}
\left\|\sum_{i=k+1}^{k+8} \eta_{i} f\right\|_{W^{1,2}\left(\bigcup_{i=k+1}^{k+4} W_{i}\right)}^{2} & =\left\|\chi_{k} f\right\|_{W^{1,2}\left(\bigcup_{i=k+1}^{k+4} W_{i}\right)}^{2} \\
= & \int_{\bigcup_{i=k+1}^{k+4} W_{i}} \chi_{k}^{2} f^{2}+\left|\left\langle\chi_{k} \nabla f+f \nabla \chi_{k}\right\rangle\right|^{2} \\
\leqslant & \int_{\bigcup_{i=k+1}^{k+4} W_{i}} f^{2}+2 \chi_{k}^{2}|\nabla f|^{2}+2 f^{2}\left|\nabla \chi_{k}\right|^{2} \\
\leqslant & \left(2 \int_{\bigcup_{i=k+1}^{k+4} W_{i}} f^{2}+|\nabla f|^{2}\right) \\
& +2\|f\|_{L^{\infty}(\Omega)}^{2} \int_{\bigcup_{i=k+1}^{k+4} W_{i}}\left|\nabla \chi_{k}\right|^{2} .
\end{aligned}
$$

It is easy to see that $\left|\nabla \chi_{k}\right|<2^{k+20}$ by estimate of $\eta_{k}$. By virtue of Minkowski codimension assumption, we obtain

$$
\left|\bigcup_{i=k+1}^{k+4} W_{i}\right|<\left|\bigcup_{i=k+1}^{\infty} W_{i}\right|<C 2^{-3 k}<C\left(2^{-k+5}\right)^{3},
$$

which in turn implies that

$$
\left\|\sum_{i=k+1}^{k+8} \eta_{i} f\right\|_{W^{1,2}\left(\bigcup_{i=k+1}^{k+4} W_{i}\right)}^{2} \leqslant 2\|f\|_{W^{1,2}\left(\bigcup_{i=k+1}^{k+4} W_{i}\right)}^{2}+C\|f\|_{L^{\infty}(\Omega)}^{2} 2^{-k} .
$$

Plug the above inequality into (2.17), we obtain

$$
\left\|\sum_{i=k+1}^{\infty} \eta_{i} f\right\|_{W^{1,2}(\Omega)}^{2} \leqslant 2\|f\|_{W^{1,2}\left(\cup_{i=k+1}^{\infty} W_{i}\right)}^{2}+C\|f\|_{L^{\infty}(\Omega)}^{2} 2^{-k} .
$$

Together with (2.15) and (2.16), the above inequality implies that

$$
\left\|H_{k}-f\right\|_{W^{1,2}(\Omega)}<\frac{1}{2} \epsilon+2\|f\|_{W^{1,2}\left(\bigcup_{i=k+1}^{\infty} W_{i}\right)}^{2}+C\|f\|_{L^{\infty}(\Omega)}^{2} 2^{-k} .
$$

Recall that $f \in W^{1,2}(\Omega \backslash \mathcal{S}),\left|\bigcup_{i=k+1}^{\infty} W_{i}\right| \rightarrow 0$ as $k \rightarrow \infty$. So we can choose $k$ large enough such that

$$
\left\|H_{k}-f\right\|_{W^{1,2}(\Omega \backslash \mathcal{S})}<\epsilon .
$$


Let $\epsilon=1 / i$, we denote the corresponding $H_{k}$ in the above inequality by $f_{i}$. Clearly, $f_{i}$ is supported on $\Omega \backslash \mathcal{S}$ and is smooth. Moreover, (2.14), consequently (2.13), follows from the above inequality.

It follows from the construction that $f_{i} \geqslant 0$ whenever $f \geqslant 0$. Also, from the construction, if $\Omega$ is bounded, supp $f_{i}$ is a compact subset of $\bar{\Omega} \backslash \mathcal{S}$.

COROLLARY 2.12 (Smooth functions with compact supports). $C_{c}^{\infty}(\mathcal{R}) \cap N^{1,2}(X)$ is dense in $N^{1,2}(X)$.

Proof. Fix $f \in N^{1,2}(X)$, without loss of generality, we may assume that $f \in$ $C^{\infty}(\mathcal{R})$ and $f$ vanishes around $\mathcal{S}$, by Proposition 2.11. Fix $x_{0} \in \mathcal{R}$ and let $r(x)=$ $d\left(x, x_{0}\right)$. For each large $k$, let $\phi_{k}=\phi(r(x)-k)$, where $\phi$ is a smooth cutoff function. $\phi \equiv 1$ on $(-\infty, 0)$ and $\phi \equiv 0$ on $(1, \infty)$. Moreover, $\left|\phi^{\prime}\right| \leqslant 2$. Note that supp $f \cap \overline{B\left(x_{0}, k+1\right)}$ is a compact subset of $B\left(x_{0}, k+2\right) \backslash \mathcal{S}$. By convolution with mollifier if necessary, we can assume $\phi_{k}$ is smooth and on supp $f \cap \operatorname{supp} \phi_{k}$, $\operatorname{supp} \phi_{k} \subset B\left(x_{0}, k+2\right), \phi_{k} \equiv 1$ on $B\left(x_{0}, k-1\right)$. Moreover, $\left|\nabla \phi_{k}\right|<4$ and $0 \leqslant$ $\phi_{k}<2$. Therefore, $\phi_{k} f \in C_{c}^{\infty}(\mathcal{R})$. It is easy to calculate

$$
\begin{aligned}
\left\|f-\phi_{k} f\right\|_{N^{1,2}(X)}^{2}= & \int_{X}\left(1-\phi_{k}\right)^{2} f^{2} d \mu+\int_{X}\left|\nabla\left\{\left(1-\phi_{k}\right) f\right\}\right|^{2} d \mu \\
\leqslant & \int_{X \backslash B\left(x_{0}, k-1\right)}\left(1-\phi_{k}\right)^{2} f^{2} d u \\
& +2 \int_{X \backslash B\left(x_{0}, k-1\right)}\left\{\left(1-\phi_{k}\right)^{2}|\nabla f|^{2}+f^{2}\left|\nabla \phi_{k}\right|^{2}\right\} d \mu \\
\leqslant & \int_{X \backslash B\left(x_{0}, k-1\right)} f^{2} d u+2 \int_{X \backslash B\left(x_{0}, k-1\right)}\left\{|\nabla f|^{2}+16 f^{2}\right\} d \mu \\
\leqslant & 33 \int_{X \backslash B\left(x_{0}, k-1\right)}\left\{|\nabla f|^{2}+f^{2}\right\} d \mu .
\end{aligned}
$$

Clearly, the right hand side of the above inequality goes to 0 as $k \rightarrow \infty$, since $f \in N^{1,2}(X)$. Therefore, every $f \in N^{1,2}(X)$ can be approximated by smooth functions with compact support.

In light of Proposition 2.11, we will define $N_{0}^{1,2}(\Omega)$ as the completion of all the functions in $C_{c}^{\infty}(\Omega \backslash \mathcal{S}) \cap N^{1,2}(X)$, under the $N^{1,2}(\Omega)$-norm. Note that a function $f$ in $N_{0}^{1,2}(\Omega)$ may not have compact support in $\Omega$. However, $\left.f\right|_{\partial \Omega}=0$, in the sense of traces.

Proposition 2.13 (Global continuous approximation). For each $f \in C_{c}(X)$, that is, a continuous function with compact support, there exists a sequence of $f_{i} \in C_{c}(X) \cap N^{1,2}(X)$ such that $\lim _{i \rightarrow \infty}\left\|f_{i}-f\right\|_{C(X)} \rightarrow 0$. 
Proof. For each $\epsilon>0, x \in X$, define $\phi_{\epsilon, x}$ to be the characteristic function of the geodesic ball $B(x, \epsilon)$. In other words, $\phi_{\epsilon, x} \equiv 1$ on $B(x, \epsilon)$ and 0 on $X \backslash B(x, \epsilon)$. Define $\psi_{\epsilon, x}$ to be $\phi_{\epsilon, x} /|B(x, \epsilon)|$. Clearly, we have

$$
\int_{X} \psi_{\epsilon, x}(y) d \mu_{y}=1
$$

Similar to Euclidean case, we define approximation functions by convoluting $f$ and $\psi_{\epsilon, \cdot}$ as follows:

$$
f_{\epsilon}(x) \triangleq\left(\psi_{\epsilon} * f\right)(x)=\int_{X} f(y) \psi_{\epsilon, x}(y) d \mu_{y} .
$$

Fix $\epsilon>0$. Suppose $x_{1}, x_{2}$ are two points in $X$ with distance $\rho \in(0, \epsilon)$. Then we calculate

$$
\begin{aligned}
& \left|f_{\epsilon}\left(x_{1}\right)-f_{\epsilon}\left(x_{2}\right)\right| \\
& \quad \leqslant \int_{X}|f|(y)\left|\psi_{\epsilon, x_{1}}(y)-\psi_{\epsilon, x_{2}}(y)\right| d \mu_{y} \\
& \quad \leqslant\|f\|_{C(X)} \int_{X}\left|\frac{\phi_{\epsilon, x_{1}}}{\left|B\left(x_{1}, \epsilon\right)\right|}-\frac{\phi_{\epsilon, x_{2}}}{\left|B\left(x_{2}, \epsilon\right)\right|}\right| d \mu_{y} \\
& \quad=\frac{\|f\|_{C(X)}}{\left|B\left(x_{1}, \epsilon\right) \| B\left(x_{2}, \epsilon\right)\right|} \int_{X}\left|\phi_{\epsilon, x_{1}}\right| B\left(x_{2}, \epsilon\right)\left|-\phi_{\epsilon, x_{2}}\right| B\left(x_{1}, \epsilon\right) \| d \mu_{y} \\
& \quad \leqslant C(n, \kappa)\|f\|_{C(X)} \epsilon^{-4 n} \int_{X}\left|\phi_{\epsilon, x_{1}}\right| B\left(x_{2}, \epsilon\right)\left|-\phi_{\epsilon, x_{2}}\right| B\left(x_{1}, \epsilon\right) \| d \mu_{y} .
\end{aligned}
$$

Notice that

$$
\begin{aligned}
\int_{X} & \left|\phi_{\epsilon, x_{1}}\right| B\left(x_{2}, \epsilon\right)\left|-\phi_{\epsilon, x_{2}}\right| B\left(x_{1}, \epsilon\right)|| d \mu_{y} \\
= & \int_{X}\left|\phi_{\epsilon, x_{1}}\left\{\left|B\left(x_{2}, \epsilon\right)\right|-\left|B\left(x_{1}, \epsilon\right)\right|\right\}+\right| B\left(x_{1}, \epsilon\right)\left|\cdot\left(\phi_{\epsilon, x_{1}}-\phi_{\epsilon, x_{2}}\right)\right| d \mu_{y} \\
\leqslant & \int_{X} \phi_{\epsilon, x_{1}}|| B\left(x_{2}, \epsilon\right)|-| B\left(x_{1}, \epsilon\right)|| d \mu_{y}+\left|B\left(x_{1}, \epsilon\right)\right| \int_{X}\left|\phi_{\epsilon, x_{1}}-\phi_{\epsilon, x_{2}}\right| d \mu_{y} \\
= & \left|B\left(x_{1}, \epsilon\right)\right|\left\{|| B\left(x_{2}, \epsilon\right)|-| B\left(x_{1}, \epsilon\right)||+\int_{X}\left|\phi_{\epsilon, x_{1}}-\phi_{\epsilon, x_{2}}\right|\right\} \\
= & \left|B\left(x_{1}, \epsilon\right)\right|\left\{|| B\left(x_{2}, \epsilon\right)|-| B\left(x_{1}, \epsilon\right)||+\left|B\left(x_{1}, \epsilon\right) \backslash B\left(x_{2}, \epsilon\right)\right|\right. \\
& \left.+\left|B\left(x_{2}, \epsilon\right) \backslash B\left(x_{1}, \epsilon\right)\right|\right\} \\
\leqslant & 2\left|B\left(x_{1}, \epsilon\right)\right|\left\{\left|B\left(x_{1}, \epsilon\right) \backslash B\left(x_{2}, \epsilon\right)\right|+\left|B\left(x_{2}, \epsilon\right) \backslash B\left(x_{1}, \epsilon\right)\right|\right\} .
\end{aligned}
$$


By Bishop-Gromov volume comparison and noncollapsing condition, we have

$$
\begin{aligned}
& \left|B\left(x_{2}, \epsilon\right) \backslash B\left(x_{1}, \epsilon\right)\right| \leqslant\left|B\left(x_{1}, \epsilon+\rho\right) \backslash B\left(x_{1}, \epsilon-\rho\right)\right| \leqslant C(n, \kappa) \epsilon^{2 n-1} \rho, \\
& \left|B\left(x_{1}, \epsilon\right) \backslash B\left(x_{2}, \epsilon\right)\right| \leqslant\left|B\left(x_{2}, \epsilon+\rho\right) \backslash B\left(x_{2}, \epsilon-\rho\right)\right| \leqslant C(n, \kappa) \epsilon^{2 n-1} \rho .
\end{aligned}
$$

Thus, for each $\rho \in(0, \epsilon)$, we have estimate

$$
\left|f_{\epsilon}\left(x_{1}\right)-f_{\epsilon}\left(x_{2}\right)\right| \leqslant \frac{C(n, \kappa)\|f\|_{C(X)}}{\epsilon} \rho,
$$

which means that the Lipschitz constant of $f_{\epsilon}$ is uniformly bounded, for each fixed $\epsilon$. In particular, $f_{\epsilon}$ belongs to $C_{c}(X) \cap N^{1,2}(X)$. It follows from (2.18) that

$$
\begin{aligned}
\left|f_{\epsilon}(x)-f(x)\right| & =\left|\int_{X}\{f(y)-f(x)\} \psi_{\epsilon, x}(y) d \mu_{y}\right| \\
& \leqslant \int_{B(x, \epsilon)}|f(y)-f(x)| \psi_{\epsilon, x}(y) d \mu_{y} \\
& \leqslant \sup _{y \in B(x, \epsilon)}|f(y)-f(x)| .
\end{aligned}
$$

Note that $f$ is uniformly continuous since $f$ is continuous and $\operatorname{supp}(f)$ is contained in a compact subset of $X$. Hence the right hand side of the above inequality converges to zero uniformly as $\epsilon \rightarrow 0$. Therefore, $\psi_{2^{-i}} * f$ is a sequence of functions in $C_{c}(X) \cap N^{1,2}(X)$ and converges to $f$ in $C(X)$-norm.

For each open set $\Omega \subset X$, there is a projection map $\pi: N^{1,2}(\Omega) \rightarrow N^{1,2}(\Omega \backslash \mathcal{S})$ in the obvious way. Note that $\Omega \backslash \mathcal{S}=\Omega \cap \mathcal{R}$ is a smooth manifold; hence $N^{1,2}(\Omega \backslash \mathcal{S})=W^{1,2}(\Omega \backslash \mathcal{S})$. In general, the map $\pi$ is not surjective. However, in our special setting, $\mathcal{S}$ has high codimension, we have much more information.

Proposition 2.14 (Identity is isometry). Suppose $\Omega$ is an open set of $X$, then the restriction map $\pi: N^{1,2}(\Omega) \rightarrow N^{1,2}(\Omega \backslash \mathcal{S})=W^{1,2}(\Omega \backslash \mathcal{S})$ is an isomorphic isometry.

Proof. It suffices to prove $\pi$ is a linear isomorphism. For simplicity, we assume $\Omega=X$. Then $\Omega \backslash \mathcal{S}=X \backslash \mathcal{S}=\mathcal{R}$.

Injectivity: suppose $\pi(f)=0$. Then $\|f\|_{L^{2}(X)}=0$ since $\mathcal{S}$ has measure zero. Due to the fact $f \in N^{1,2}(X),\|f\|_{L^{2}(X)}=0$ implies that $\|f\|_{N^{1,2}(X)}=0$. Therefore, $f$ is the zero element in $N^{1,2}(X)$.

Surjectivity: for every $0 \neq \tilde{f} \in W^{1,2}(\mathcal{R})$, from the proof of Proposition 2.11, there is a sequence of smooth functions $\tilde{f}_{i}$ supported on $\mathcal{R}$ such that

$$
\left\|\tilde{f}_{i}-\tilde{f}\right\|_{W^{1,2}(\mathcal{R})} \rightarrow 0 .
$$


In particular, $\tilde{f}_{i}$ is a Cauchy sequence in $W^{1,2}(\mathcal{R})$. Since

$$
\left\|\tilde{f}_{i}-\tilde{f}\right\|_{N^{1,2}(X)}=\left\|\tilde{f}_{i}-\tilde{f}\right\|_{W^{1,2}(\mathcal{R})},
$$

it is clear that $\tilde{f}_{i}$ is a Cauchy sequence in $N^{1,2}(X)$. Therefore, there is a function $f \in N^{1,2}(X)$, as the limit of $\tilde{f}_{i}$, by completeness of $N^{1,2}(X)$. After we obtain $f$, it is clear that $\left\|f_{i}-f\right\|_{N^{1,2}(X)} \rightarrow 0$, which forces that

$$
\left\|f_{i}-\pi(f)\right\|_{W^{1,2}(\mathcal{R})} \rightarrow 0 .
$$

Therefore, $\pi(f)=\tilde{f}$.

In light of Proposition 2.14, we can regard $N^{1,2}(X)$ as the same Banach space as $W^{1,2}(\mathcal{R})$. However, $W^{1,2}(\mathcal{R})$ is a Hilbert space. This induces a natural inner product structure on $W^{1,2}(\mathcal{R})$ as follows:

$$
\left\langle\left\langle f_{1}, f_{2}\right\rangle\right\rangle=\int_{\mathcal{R}}\left\{\pi\left(f_{1}\right) \pi\left(f_{2}\right)+\left\langle\nabla \pi\left(f_{1}\right), \nabla \pi\left(f_{2}\right)\right\rangle\right\} d \mu, \quad \forall f_{1}, f_{2} \in N^{1,2}(X) .
$$

For simplicity of notation, we shall not distinguish between $f$ and $\pi(f)$. Under this convention, we have

$$
\left\langle\left\langle f_{1}, f_{2}\right\rangle\right\rangle=\int_{\mathcal{R}}\left\{f_{1} f_{2}+\left\langle\nabla f_{1}, \nabla f_{2}\right\rangle\right\} d \mu, \quad \forall f_{1}, f_{2} \in N^{1,2}(X) .
$$

Therefore, $N^{1,2}(X)$ is isomorphic to $W^{1,2}(\mathcal{R})$ as a Hilbert space. For every $f_{1}$, $f_{2} \in N^{1,2}(X)$, we define a nonnegative, symmetric, bilinear form $\mathscr{E}$ as follows

$$
\mathscr{E}\left(f_{1}, f_{2}\right) \triangleq \int_{\mathcal{R}}\left\langle\nabla f_{1}, \nabla f_{2}\right\rangle d \mu
$$

We want to show that $\mathscr{E}$ is a Dirichlet form. Actually, it is clear that $\|f\|_{N^{1,2}(X)}^{2}=$ $\|f\|_{L^{2}(X)}^{2}+\mathscr{E}(f, f)$. Since $N^{1,2}(X)$ is complete, we know that $\mathscr{E}$ is closed by definition. On the other hand, since $W^{1,2}(\mathcal{R})$ is dense in $L^{2}(\mathcal{R})=L^{2}(X), \mathcal{S}$ has measure zero, it follows directly that $N^{1,2}(X)$ is dense in $L^{2}(X)$. Furthermore, it is clear that

$$
\mathscr{E}(\min \{1, \max \{0, f\}\}, \min \{1, \max \{0, f\}\}) \leqslant \mathscr{E}(f, f), \quad \forall f \in N^{1,2}(X) .
$$

Therefore, $\mathscr{E}$ is a closed, nonnegative, symmetric, bilinear form on $N^{1,2}(X)$, which is a dense subspace of $L^{2}(X)$, with unit contraction property (2.20). It follows from a standard definition (see [24] for definition of Dirichlet form) that $\mathscr{E}$ is a Dirichlet form. Not surprisingly, this Dirichlet form $\mathscr{E}$ is much better than general Dirichlet form since the underlying space $X$ has rich geometry. In fact, 
suppose $u \in N_{0}^{1,2}(\Omega)$ for some open set $\Omega \subset X$, it is clear that $u \equiv 0$ on $\Omega$ if and only if $\mathscr{E}(u, u)=0$. This means that $\mathscr{E}$ is irreducible by direct definition. Also, for every constant $c$, we have $\mathscr{E}(u, v)=0$, whenever $v \equiv c$ in a neighborhood of the support set of $u$. This means that $\mathscr{E}$ is strongly local. Furthermore, it follows from Corollary 2.12 that $N^{1,2}(X) \cap C_{c}(X)$ is dense in $N^{1,2}(X)$ with $N^{1,2}$-norm. On the other hand, Proposition 2.13 implies that $N^{1,2}(X) \cap C_{c}(X)$ is dense in $C_{c}(X)$ with uniform supreme norm. Consequently, $N^{1,2}(X) \cap C_{c}(X)$ is a core of $\mathscr{E}$ and $\mathscr{E}$ is a regular Dirichlet form, following from the definition verbatim. Putting all the above information together, we obtain the following property.

Proposition 2.15 (Existence of excellent Dirichlet form). On the Hilbert space $L^{2}(X)$, there exists a Dirichlet form $\mathscr{E}$ defined on a dense subspace $N^{1,2}(X) \subset$ $L^{2}(X)$, by formula (2.19). Furthermore, the Dirichlet form $\mathscr{E}$ is irreducible, strongly local and regular.

With respect to the Dirichlet form $\mathscr{E}$, one can obtain much geometric and analytic information. A good reference is the nice paper [32], by Koskela and Zhou. We now focus on some elementary properties. Note that there is a unique generator (see [24, Ch. 1]) of $\mathscr{E}$, which we denote by $\mathcal{L}$. In other words, $\mathcal{L}$ is a self-adjoint and nonpositive definite operator in $L^{2}(X)$ with domain $\operatorname{Dom}(\mathcal{L})$ which is dense in $N^{1,2}(X)$ such that

$$
\mathscr{E}(f, h)=-\int_{X} h \cdot \mathcal{L} f d \mu, \quad \forall f \in \operatorname{Dom}(\mathcal{L}), h \in N^{1,2}(X) .
$$

Note that $C_{c}^{\infty}(\mathcal{R})$ is a dense subset of $\operatorname{Dom}(\mathcal{L})$. Suppose $f \in C_{c}^{\infty}(\mathcal{R}), h \in$ $N^{1,2}(X)=N^{1,2}(\mathcal{R})$, it is clear that

$$
\mathscr{E}(f, h)=\int_{\mathcal{R}}\langle\nabla f, \nabla h\rangle d \mu=-\int_{X} h \cdot \Delta f d \mu .
$$

Therefore, $\mathcal{L}$ is nothing but the extension of the classical Laplacian operator, with domain as the largest dense subset of $N^{1,2}(X)$ such that the integration by parts, that is, Equation (2.21), holds. For this reason, we shall just denote $\mathcal{L}$ by $\Delta$ in the future.

Based on the generator operator $\Delta$, there is an associated heat semigroup $\left(P_{t}\right)_{t \geqslant 0}=\left(e^{t \Delta}\right)_{t \geqslant 0}$, which acts on $L^{2}(X)$ with the following properties (see [24, Ch. 1]).

- Semigroup: $P_{0}=\mathrm{Id} ; P_{t} \circ P_{s}=P_{t+s}$, for every $t, s \geqslant 0$.

- Generator: $\lim _{t \rightarrow 0^{+}}\left\|(1 / t)\left(P_{t} f-f\right)-\Delta f\right\|_{L^{2}(X)}=0$, for every $f \in L^{2}(X) \cap$ $\operatorname{Dom}(\Delta)$. 
- $L^{2}$-contractive: $\left\|P_{t} f\right\|_{L^{2}(X)}^{2} \leqslant\|f\|_{L^{2}(X)}^{2}$, for every $f \in L^{2}(X), t>0$.

- Strong continuous: $\lim _{t \rightarrow 0^{+}}\left\|P_{t} f-f\right\|_{L^{2}(X)}=0$, for every $f \in L^{2}(X)$.

- Markovian: $\left\|P_{t} f\right\|_{L^{\infty}(X)} \leqslant\|f\|_{L^{\infty}(X)}$, for every $f \in L^{2}(X) \cap L^{\infty}(X), t>0$.

- Heat solution: $\Delta P_{t} f=(\partial / \partial t) P_{t} f$, for every $f \in L^{2}(X)$ and $t>0$.

The above properties are well known in semigroup theory on Banach spaces (see [22, Section 7.4]). Actually, for every $f \in L^{2}(X)$, one can also show that $P_{t} f$ is the unique square-integrable solution with initial value $f$ (see [41, Proposition 1.2] and references therein). We call $\left(P_{t}\right)_{t \geqslant 0}$ the heat semigroup as usual. Associated with this heat semigroup, there exists a nonnegative kernel function, or fundamental solution, $p(t, x, y)$, such that

$$
P_{t}(f)(y)=\int_{X} f(x) p(t, x, y) d \mu_{x}, \quad \forall f \in L^{2}(X), t>0 .
$$

Moreover, $p$ satisfies the symmetry $p(t, x, y)=p(t, y, x)$. Interested readers are referred to Proposition 2.3 and the discussion in Section 2.4(C) of [41] for more detailed information. As usual, we call $p(t, x, y)$ as the heat kernel.

Definition 2.16. Suppose $u \in N_{\text {loc }}^{1,2}(\Omega)$. Define

$$
\int_{\Omega} \varphi \Delta u \triangleq-\mathscr{E}(u, \varphi)
$$

for every $\varphi \in N_{c}^{1,2}(\Omega)$, that is, $\varphi \in N^{1,2}(\Omega)$ and has compact support set in $\Omega$. Similarly, (2.23) can be applied if $u \in N^{1,2}(\Omega)$ and $\varphi \in N_{0}^{1,2}(\Omega)$.

Suppose $u \in N_{\text {loc }}^{1,2}(\Omega) \cap C^{2}(\Omega \backslash \mathcal{S})$, then $\left.\Delta u\right|_{\Omega \backslash \mathcal{S}}$ is a continuous function. By taking value $\infty$ on $\mathcal{S}$, we can regard $\Delta u$ as an extended function on $\Omega$. Suppose $\Delta u \in L_{\mathrm{loc}}^{2}(\Omega)$, then for every smooth test function $\varphi_{i} \in N_{c}^{1,2}(\Omega)$, we have

$$
\int_{\Omega \backslash \mathcal{S}} \varphi_{i} \Delta u=-\int_{\Omega \backslash \mathcal{S}}\left\langle\nabla u, \nabla \varphi_{i}\right\rangle .
$$

Let $\varphi$ be the limit of $\varphi_{i}$ in $N^{1,2}(\Omega)$. Taking limit of the above equation shows that

$$
\int_{\Omega} \varphi \Delta u=-\int_{\Omega}\langle\nabla u, \nabla \varphi\rangle .
$$

Therefore, whenever $u \in N_{\text {loc }}^{1,2}(\Omega) \cap C^{2}(\Omega \backslash \mathcal{S})$ and the classical $\Delta u$ is in $L_{\text {loc }}^{2}(\Omega)$, we see that the LHS and RHS of (2.23) hold in the classical sense. Similar argument applies if $u \in N^{1,2}(\Omega) \cap C^{2}(\Omega \backslash \mathcal{S}), \Delta u \in L^{2}(\Omega), \varphi \in N_{0}^{1,2}(\Omega)$. Therefore, Definition 2.16 is justified. 
Now we assume $u \in N_{c}^{1,2}(\Omega)$. Then in the weak sense, for every $\varphi \in N_{c}^{1,2}(\Omega)$, we can define $\int_{\Omega} \varphi \Delta u$. It is not hard to see that $\int_{\Omega} \varphi \Delta u$ makes sense even if $\varphi$ is in $N^{1,2}(\Omega)$ only. In fact, let $\chi$ be a cutoff function with value 1 on $\Omega^{\prime}$ and vanishes around $\partial \Omega$, where $\Omega^{\prime}$ contains the support of $u$. By Definition 2.16, we have

$$
\begin{aligned}
\int_{\Omega}(\chi \varphi) \Delta u & =-\mathscr{E}(u, \chi \varphi)=-\int_{\Omega}\langle\nabla u, \nabla(\chi \varphi)\rangle=-\int_{\Omega^{\prime}}\langle\nabla u, \nabla \varphi\rangle \\
& =-\int_{\Omega}\langle\nabla u, \nabla \varphi\rangle=-\mathscr{E}(u, \varphi) .
\end{aligned}
$$

The above calculation does not depend on the particular choice of $\chi$. Consequently, we can define $\int_{\Omega} \varphi \Delta u$ as $-\mathscr{E}(u, \varphi)$. Summarizing the above discussion, we have the following property.

Proposition 2.17 (Integration by parts). Suppose $\Omega$ is a domain in $X, f_{1} \in$ $N_{c}^{1,2}(\Omega), f_{2} \in N^{1,2}(\Omega)$. Then we have

$$
\int_{\Omega} f_{2} \Delta f_{1} d \mu=-\int_{\Omega}\left\langle\nabla f_{1}, \nabla f_{2}\right\rangle d \mu=\int_{\Omega} f_{1} \Delta f_{2} d \mu .
$$

Furthermore, if $f_{2} \in N^{1,2}(\Omega) \cap C^{2}(\Omega \backslash \mathcal{S})$ and $\Delta f_{2} \in L^{2}(\Omega \backslash \mathcal{S})$ as a classical function, then we can understand the integral $\int_{\Omega} f_{1} \Delta f_{2} d \mu=\int_{\Omega \backslash \mathcal{S}} f_{1} \Delta f_{2} d \mu$ in the classical sense. Similarly, if $f_{1} \in N_{c}^{1,2}(\Omega) \cap C^{2}(\Omega \backslash \mathcal{S}), \Delta f_{1} \in L^{2}(\Omega \backslash \mathcal{S})$ as a classical function, then $\int_{\Omega} f_{2} \Delta f_{1} d \mu=\int_{\Omega \backslash \mathcal{S}} f_{2} \Delta f_{1} d \mu$ can be understood in the classical sense. If both $f_{1}$ and $f_{2}$ locate in $N_{0}^{1,2}(\Omega)$, then (2.24) also holds.

Definition 2.18. Suppose $u \in N_{\mathrm{loc}}^{1,2}(\Omega), f \in L_{\mathrm{loc}}^{2}(\Omega)$, we say $\Delta u \geqslant f$ in the weak sense if

$$
\int_{\Omega}(-\Delta u+f) \varphi=\mathscr{E}(u, \varphi)+\int_{\Omega} f \varphi \leqslant 0
$$

for every nonnegative test function $\varphi \in N_{c}^{1,2}(\Omega)$. We call $u$ subharmonic if $\Delta u \geqslant 0$ in the weak sense. We call $u$ superharmonic if $-u$ is subharmonic. We call $u$ harmonic if $u$ is both subharmonic and superharmonic.

Due to Proposition 2.11, for a $u \in N_{\text {loc }}^{1,2}(\Omega)$, in order to check (2.25) for all $\varphi \in$ $N_{c}^{1,2}(\Omega)$, it suffices to check all smooth nonnegative test functions with supports in $\Omega \backslash \mathcal{S}$. It is important to notice that the restriction of $\Delta$ on $\mathcal{R}$ is the classical Laplacian on Riemannian manifold. In fact, if a function $u$ is harmonic in the above sense, then $\left.u\right|_{\mathcal{R}}$ is harmonic function in the distribution sense. By standard improving regularity theory of elliptic equations, we know our $u$ is smooth and $\Delta u=0$ in the classical sense. 
Similarly, one can follow the standard route to define heat solution (subsolution, supersolution) for the heat operator $\square=((\partial / \partial t)-\Delta)$ in the weak sense. We leave these details to interested readers. It is quite clear that a weak heat solution is a smooth function when restricted on $\mathcal{R} \times(0, T]$, by standard improving regularity theory of heat equations (see [22, Ch. 7]).

2.3. Harmonic functions and heat flow solutions on model space. Suppose $K$ is a compact subset of $\Omega \backslash \mathcal{S}$, it is clear that $K$ is also a compact subset of $\Omega$. However, the converse is not true. If $K$ is a compact subset of $\Omega$, then $K \backslash \mathcal{S}$ may not be a compact subset of $\Omega \backslash \mathcal{S}$. For this reason, we see that $N_{\text {loc }}^{1,2}(\Omega) \subset$ $N_{\text {loc }}^{1,2}(\Omega \backslash \mathcal{S})$ and not equal if $\mathcal{S} \neq \emptyset$, even if $\mathcal{S}$ has very high codimension. However, if we restrict our attention only to bounded subharmonic functions, then the above difference will vanish.

Proposition 2.19 (Extension of bounded subharmonic functions). Suppose $\Omega$ is a bounded open domain in $X, u$ is a bounded subharmonic function on $\Omega \backslash \mathcal{S}$. Then $u \in N_{\mathrm{loc}}^{1,2}(\Omega)$ and it is subharmonic on $\Omega$.

Proof. It suffices to prove that $u \in N_{\text {loc }}^{1,2}(\Omega)$. Note that by definition, we only have $u \in N_{\text {loc }}^{1,2}(\Omega \backslash \mathcal{S})$. It is not clear whether $u \in N_{\text {loc }}^{1,2}(\Omega)$. In fact, for each small $r>0$, one can construct a Lipschitz cutoff function

$$
\chi(x)=\phi\left(\frac{d(x, \mathcal{S})}{r}\right)
$$

where $\phi$ is a cutoff function on $[0, \infty)$ which is equivalent to 1 on $[0,1], 0$ on $[2, \infty)$, and $\left|\phi^{\prime}\right| \leqslant 2$. By the assumption of Minkowski codimension of $\mathcal{S}$, we have

$$
|\Omega \cap \operatorname{supp} \chi| \leqslant C r^{3}, \quad \int_{\Omega}|\nabla \chi|^{2} \leqslant C r^{-2} \int_{\Omega \cap\{\nabla \chi \neq 0\}} \chi^{2} \leqslant C r .
$$

Fix a relatively compact subset $\Omega^{\prime} \subset \Omega$, we can find a cutoff function $\eta$ which is identically 1 on $\Omega^{\prime}$ and vanishes around $\partial \Omega$. Moreover, $|\nabla \eta| \leqslant C$, which depends on $\Omega^{\prime}$ and $\Omega$.

By adding a constant if necessary, we can assume $u \geqslant 0$. Note that $u$ is subharmonic on $\Omega \backslash \mathcal{S}, u \eta^{2}(1-\chi)^{2}$ can be chosen as a test function. It follows from the definition that

$$
\begin{aligned}
0 & \leqslant \int_{\Omega \backslash \mathcal{S}}(\Delta u) u \eta^{2}(1-\chi)^{2}=-\int_{\Omega \backslash \mathcal{S}}\left\langle\nabla u, \nabla\left(u \eta^{2}(1-\chi)^{2}\right)\right\rangle \\
& =-\int_{\Omega \backslash \mathcal{S}}|\nabla u|^{2} \eta^{2}(1-\chi)^{2}+\int_{\Omega \backslash \mathcal{S}} u\left\langle\nabla u,-2(1-\chi)^{2} \eta \nabla \eta+2 \eta^{2}(1-\chi) \nabla \chi\right\rangle .
\end{aligned}
$$


Note that $u, \eta, \nabla \eta$ are bounded. Then the Hölder inequality applies.

$$
\begin{aligned}
\frac{1}{2} \int_{\Omega \backslash \mathcal{S}}|\nabla u|^{2} \eta^{2}(1-\chi)^{2} & \leqslant C+C \int_{\Omega \backslash \mathcal{S}} \eta^{2}(1-\chi)|\nabla u||\nabla \chi| \\
& \leqslant C+\frac{1}{4} \int_{\Omega \backslash \mathcal{S}}|\nabla u|^{2} \eta^{2}(1-\chi)^{2}+C \int_{\Omega \backslash \mathcal{S}} \eta^{2}|\nabla \chi|^{2} .
\end{aligned}
$$

Recall the definition of $\chi$ in (2.26) and estimate (2.27). Let $r \rightarrow 0$, the above inequality yields that

$$
\int_{\Omega \backslash \mathcal{S}}|\nabla u|^{2} \eta^{2} \leqslant C
$$

which forces that $\int_{\Omega^{\prime} \backslash \mathcal{S}}|\nabla u|^{2} \leqslant C$. Hence $u \in W^{1,2}\left(\Omega^{\prime} \backslash \mathcal{S}\right)$ since $u$ is bounded. This is the same to say $u \in N^{1,2}\left(\Omega^{\prime}\right)$. By the arbitrary choice of $\Omega^{\prime}$, we have proved that $u \in N_{\text {loc }}^{1,2}(\Omega)$.

We now move on to the discussion of heat kernels.

Proposition 2.20 (Heat Kernel estimates). There exists a unique heat kernel $p(t, x, y)$ of $X$, with respect to the Dirichlet form $\mathscr{E}=\langle\nabla \cdot, \nabla \cdot\rangle$. Moreover, $p(t, x, y)$ satisfies the following properties.

- Stochastically completeness. In other words, we have

$$
\int_{X} p(t, x, y) d \mu_{x}=1
$$

for every $x \in X$.

- The Gaussian estimate holds. In other words, there exists a constant $C$ depending only on $n, \kappa$ such that

$$
\frac{1}{C} t^{-n} e^{-\left(d^{2}(x, y)\right) / 3 t} \leqslant p(t, x, y) \leqslant C t^{-n} e^{-\left(d^{2}(x, y)\right) / 5 t}
$$

for every $x, y \in X$ and $t>0$.

- For each positive integer $j$, there is a constant $C=C(n, \kappa, j)$ such that

$$
\left|\left(\frac{\partial}{\partial t}\right)^{j} p(t, x, y)\right| \leqslant C t^{-n-j} e^{-\left(d^{2}(x, y)\right) / 5 t}
$$

for every $x, y \in X$ and $t>0$. 
Proof. Since $X$ satisfies the doubling property and has a uniform $(1,2)$-Poincaré constant, by Corollary 2.4 and Proposition 2.7, the existence of the heat Kernel follows from the work of Sturm (see [41, Proposition 2.3]). The uniqueness of the heat kernel follows from the uniqueness of the heat semigroup. The stochastic completeness is guaranteed by the doubling property, see Theorem 4 and the following remarks of [40].

The Gaussian estimate follows from Corollaries 4.2 and 4.10 in Sturm's paper [42], where $C$ depends on the volume doubling condition and the $(1,2)$ Poincaré constant.

The heat kernel derivative estimate, inequality (2.29), follows from [41, Corollary 2.7], whose proof follows the same line as [37, Theorem 6.3].

Based on the parabolic Harnack inequality established in Sturm [42], one has Hölder continuity of heat solutions (see [42, Proposition 3.1]). Therefore, the heat kernel function $p$ has a Hölder continuous representative on its domain. Much more can be said in the current setting. Fix $x \in X$, when restricted on $\mathcal{R} \times(0, \infty)$, $p(t, x, y)$ is clearly a smooth function. Therefore, (2.28) and (2.29) actually hold true everywhere away from $\mathcal{S}$. Note that $\Delta p=(\partial / \partial t) p$ clearly locates in $L^{2}(X) \cap$ $C^{\infty}(\mathcal{R})$. Hence integration by parts (Proposition 2.17) applies. Then by standard radial cutoff function construction and direct calculation, Proposition 2.20 yields the following estimates immediately.

COROLlary 2.21 (Off-diagonal integral estimates of heat kernel). For every $r>0, t>0$, and $x_{0} \in X$, we have

$$
\begin{gathered}
\int_{X \backslash B\left(x_{0}, 2 r\right)} p^{2} d \mu_{x}<C t^{-n} e^{-r^{2} / 5 t} \\
\int_{X \backslash B\left(x_{0}, 2 r\right)}\left|\nabla p\left(t, x, x_{0}\right)\right|^{2} d \mu_{x}<C\left(\frac{1}{t}+\frac{1}{r^{2}}\right) t^{-n} e^{-r^{2} / 5 t},
\end{gathered}
$$

for some $C=C(n, \kappa)$. Consequently, we have

$$
\int_{0}^{t} \int_{X \backslash B\left(x_{0}, 2 r\right)}\left(p^{2}+|\nabla p|^{2}\right) d \mu_{x} d s<C \int_{0}^{t}\left(1+\frac{1}{s}+\frac{1}{r^{2}}\right) s^{-n} e^{-r^{2} / 5 s} d s .
$$

By virtue of Proposition 2.11, smooth functions are dense in $N^{1,2}(X)$. Then it is easy to see that $(X, g, d \mu)$, together with the heat process, has nonnegative Ricci curvature in the sense of Bakry-Emery, that is, $X \in C D(0, \infty)$ by the notation of Bakry-Emery (see [3, 4]). The following Proposition is nothing but part of [3, Proposition 2.1]. The rigorous proof is tedious and is postponed in the appendix. 
Proposition 2.22 (Weighted-Sobolev inequality). For every function $f \in$ $N^{1,2}(X)$, every $t>0$ and every $y \in X$, we have

$$
\begin{aligned}
& \int_{X} f^{2}(x) p(t, x, y) d \mu_{x}-\left(\int_{X} f(x) p(t, x, y) d \mu_{x}\right)^{2} \\
& \quad \leqslant 2 t \int_{X}|\nabla f|^{2}(x) p(t, x, y) d \mu_{x} .
\end{aligned}
$$

In other words, for every $t>0$, with respect to the probability measure $p(t, x, y) d \mu_{x}, L^{2}$-Sobolev inequality holds with the uniform Sobolev constant $1 / 2 t$.

On a Riemannian manifold with proper geometry bound, the heat kernel can be regarded as a solution starting from a $\delta$-function. This property also holds for every $X \in \widetilde{\mathscr{K} \mathscr{S}}(n, \kappa)$.

PROPOSITION 2.23 ( $\delta$-function property of heat kernel). Suppose $w$ is a function on $[0, t] \times X$, differentiable along the time direction, $w(s, \cdot) \in N_{c}^{1,2}(X)$ for each $s \in[0, t]$. Moreover, we assume $\lim \sup _{s \rightarrow 0^{+}}\|w(s, \cdot)\|_{L^{1}(X)}<\infty$, w is continuous at $\left(0, x_{0}\right)$. Then we have

$$
\begin{aligned}
& -w\left(0, x_{0}\right)+\int_{X} w(t, x) p\left(t, x, x_{0}\right) d \mu_{x} \\
& =\int_{0}^{t} \int_{X}\left\{\left(\frac{\partial}{\partial s}+\Delta\right) w(s, x)\right\} p\left(s, x, x_{0}\right) d \mu_{x} d s .
\end{aligned}
$$

Consequently, Equation (2.34) holds for functions $w(s, x)+a(s)$ where a is a differentiable function of time.

Proof. Clearly, (2.34) holds if $w(s, \cdot) \equiv a(s)$. Therefore, it suffices to show (2.34) when $w(s, \cdot) \in N_{c}^{1,2}(X)$ for each $s$. For simplicity of notation, we denote $p\left(t, x, x_{0}\right)$ by $p$ and assume $d \mu_{x}$ is the default measure. It follows from integration by parts that

$$
\begin{aligned}
\frac{d}{d t} \int_{X} w p & =\int_{X}\left\{\left(\frac{\partial}{\partial t}+\Delta\right) w\right\} p+\int_{X} w\left\{\left(\frac{\partial}{\partial t}-\Delta\right) p\right\} \\
& =\int_{X}\left\{\left(\frac{\partial}{\partial t}+\Delta\right) w\right\} p .
\end{aligned}
$$


For each $\epsilon>0$, we can find $\delta$ small enough such that $\left|w(s, x)-w\left(0, x_{0}\right)\right|<\epsilon$ whenever $0<s<\delta^{2}$ and $d\left(x, x_{0}\right)<\delta$. Then the heat kernel estimate implies that

$$
\begin{aligned}
\mid- & w\left(0, x_{0}\right)+\lim _{t \rightarrow 0^{+}} \int_{X} w(t, x) p\left(t, x, x_{0}\right) d \mu_{x} \mid \\
= & \left|\lim _{t \rightarrow 0^{+}} \int_{X}\left\{w(t, x)-w\left(0, x_{0}\right)\right\} p\left(t, x, x_{0}\right) d \mu_{x}\right| \\
\leqslant & \lim _{t \rightarrow 0^{+}}\left\{\left|w\left(0, x_{0}\right)\right| \int_{X \backslash B\left(x_{0}, \delta\right)} p\left(t, x, x_{0}\right) d \mu_{x}\right. \\
& \left.+\int_{X \backslash B\left(x_{0}, \delta\right)}|w(t, x)| p\left(t, x, x_{0}\right) d \mu_{x}+\epsilon\right\} \\
\leqslant &
\end{aligned}
$$

By arbitrary choice of $\epsilon$, we have

$$
\lim _{t \rightarrow 0^{+}} \int_{X} w(t, x) p\left(t, x, x_{0}\right) d \mu_{x}=w\left(0, x_{0}\right) .
$$

Plugging this relationship into the integrals of previous equation, we obtain (2.34).

Based on the excellent properties of heat kernels, from Propositions 2.20 to 2.23, we are ready to generalize the celebrated Cheng-Yau estimate (see [19]) to our setting. We basically follow the paper [31]. However, due to the essential importance of this estimate and the excellent geometry of our underlying space, we write down a simplified proof here.

Proposition 2.24 (Cheng-Yau type gradient estimate). Suppose $\Omega=B\left(x_{0}\right.$, $4 r$ ) for some $r>0$ and $x_{0} \in \mathcal{R}$. Suppose $u \in L^{\infty}(\Omega) \cap N^{1,2}(\Omega)$ and $u$ satisfies the equation

$$
\Delta u=h
$$

for some $h \in C^{1 / 2}(\Omega)$. Then we have

$$
|\nabla u|\left(x_{0}\right) \leqslant \frac{C}{r}\left(\|u\|_{L^{\infty}(\Omega)}+r^{5 / 2}[h]_{C^{1 / 2}(\Omega)}+r^{2}\left|h\left(x_{0}\right)\right|\right)
$$

for a constant $C=C(n, \kappa)$, where

$$
[h]_{C^{1 / 2}(\Omega)}=\sup _{x, y \in \Omega} \frac{|h(x)-h(y)|}{d^{1 / 2}(x, y)} .
$$


Proof. Without loss of generality, we assume $r=1$ and $\|u\|_{L^{\infty}(\Omega)}=1$. Let $\chi$ be a Lipschitz cutoff function such that $\chi \equiv 1$ on $B\left(x_{0}, 1\right)$ and vanishes outside $B\left(x_{0}, 2\right)$ such that $|\nabla \chi| \leqslant 2$. For each $t>0$, define

$$
\begin{gathered}
a(t) \triangleq P_{t}(u \chi)\left(x_{0}\right), \quad J(t) \triangleq \frac{1}{t} \int_{0}^{t} \int_{X}|\nabla w(s, x)|^{2} p\left(s, x, x_{0}\right) d \mu_{x} d s, \\
w(t, x) \triangleq u(x) \chi(x)-a(t), \quad J(0) \triangleq \lim _{t \rightarrow 0^{+}} J(t)=\left|\nabla w\left(0, x_{0}\right)\right|^{2}=\left|\nabla u\left(x_{0}\right)\right|^{2} .
\end{gathered}
$$

Recall that the operator $P_{t}$ is defined in (2.22). From the definition of $w(t, x)$, it is clear that $\int_{X} w(t, x) p\left(t, x, x_{0}\right) d \mu_{x}=0$. Applying (2.33), we have

$$
\begin{aligned}
\int_{X} w^{2}(t, x) p\left(t, x, x_{0}\right) d \mu_{x} & \leqslant 2 t \int_{X}|\nabla w|^{2} p\left(t, x, x_{0}\right) d \mu_{x}, \\
|a(t)|=\left|\int_{X} u \chi p\left(t, x, x_{0}\right) d \mu_{x}\right| & \leqslant \int_{X}|u \chi| p\left(t, x, x_{0}\right) d \mu_{x} \\
& \leqslant \int_{X} p\left(t, x, x_{0}\right) d \mu_{x} \leqslant 1, \\
|w(t, x)|=|u(x) \chi(x)-a(t)| & \leqslant|u(x) \chi(x)|+|a(t)| \leqslant 2, \quad \forall x \in X .
\end{aligned}
$$

It follows from the definition of $J$ that

$$
\left|\nabla u\left(x_{0}\right)\right|^{2}=J(0)=-\int_{0}^{1} J^{\prime}(t) d t+J(1) .
$$

However, in light of (2.37), we have

$$
\begin{aligned}
J^{\prime}(t) & =-\frac{1}{t} J(t)+\frac{1}{t} \int_{X}|\nabla w|^{2} p\left(t, x, x_{0}\right) d \mu_{x} \\
& \geqslant-\frac{1}{t} J(t)+\frac{1}{2 t^{2}} \int_{X} w^{2}(t, x) p\left(t, x, x_{0}\right) d \mu_{x} \\
& =\frac{1}{t^{2}}\left(-t J(t)+\frac{1}{2} \int_{X} w^{2}(t, x) p\left(t, x, x_{0}\right) d \mu_{x}\right)=\frac{F(t)}{t^{2}},
\end{aligned}
$$

where we used the definition $F(t) \triangleq-t J(t)+\frac{1}{2} \int_{X} w^{2}(t, x) p\left(t, x, x_{0}\right) d \mu_{x}$. Therefore, by the previous inequalities, Equation (2.40) can be rewritten as

$$
\left|\nabla u\left(x_{0}\right)\right|^{2} \leqslant-\int_{0}^{1} \frac{F(s)}{s^{2}} d s-F(1)+\frac{1}{2} \int_{X} w^{2} p \leqslant 2-\int_{0}^{1} \frac{F(s)}{s^{2}} d s-F(1) .
$$

Therefore, $\left|\nabla u\left(x_{0}\right)\right|$ follows from the estimate of $F(t)$. 
We now focus on the estimate of $F(t)$. Applying (2.34) to $w^{2}$, we obtain

$$
\int_{0}^{t} \int_{X}\left\{\left(\frac{\partial}{\partial s}+\Delta\right) w^{2}(s, x)\right\} p\left(s, x, x_{0}\right) d \mu_{x} d s=\int_{X} w^{2}(t, x) p\left(t, x, x_{0}\right) d \mu_{x},
$$

since $w\left(0, x_{0}\right)=0$. Note that the application of (2.34) can be justified. Actually, from its definition, $w^{2}(x, t)=u \chi(u \chi-2 a(t))+a^{2}(t)$. The first part of the right hand side of this equation is a function in $N_{c}^{1,2}(X)$ for each $t$. It is Lipschitz continuous around $\left(0, x_{0}\right)$, since $x_{0}$ is a smooth point and the standard improving regularity theory of elliptic functions applies here. The second part is a differentiable function of time. Therefore, (2.34) applies for $w^{2}$. On the other hand, the fact that $u$ is a weak solution of (2.35) implies that

$$
|\nabla w|^{2}=\frac{1}{2} \Delta w^{2}-w \Delta w=\frac{1}{2} \Delta w^{2}-w(u \Delta \chi+\chi h+2\langle\nabla u, \nabla \chi\rangle)
$$

in the weak sense. Plugging the above equation and (2.42) into the definition of $J(t)$, we have

$$
\begin{aligned}
t J(t)= & \int_{0}^{t} \int_{X}\left\{\frac{1}{2}\left(\frac{\partial}{\partial s}+\Delta\right) w^{2}-w(u \Delta \chi+\chi h+2\langle\nabla u, \nabla \chi\rangle)\right\} \\
& \times p\left(s, x, x_{0}\right) d \mu_{x} d s \\
= & \int_{0}^{t} \int_{X}\left\{\frac{1}{2} w^{2}-w(u \Delta \chi+\chi h+2\langle\nabla u, \nabla \chi\rangle)\right\} p\left(s, x, x_{0}\right) d \mu_{x} d s .
\end{aligned}
$$

For the simplicity of notation, we will denote $p\left(s, x, x_{0}\right)$ by $p$ only. Also, we will drop integration elements when they are clear. From the definition of $F(t)$, the above equation can be written as

$$
F(t)-\int_{0}^{t} \int_{X} w \chi h p=\int_{0}^{t} \int_{X} w(u \Delta \chi+2\langle\nabla u, \nabla \chi\rangle) p
$$

Recall that $w=u \chi-a$ and $\nabla w=u \nabla \chi+\chi \nabla u$. Integrating by parts gives us

$$
\begin{aligned}
F(t)-\iint w \chi h p & =\iint\langle-u p \nabla w-u w \nabla p+p w \nabla u, \nabla \chi\rangle \\
& =\iint\langle-a p \nabla u-u w \nabla p, \nabla \chi\rangle-p u^{2}|\nabla \chi|^{2} .
\end{aligned}
$$


By (2.38) and (2.39), we have $|a| \leqslant 1$ and $|u w| \leqslant 2$. By the choice of $\chi$ and the Hölder inequality, it is clear that

$$
\begin{aligned}
& \left|F(t)+\int_{0}^{t} \int_{X}\left(u^{2}|\nabla \chi|^{2}-w \chi h\right) p\right| \leqslant 2 \int_{0}^{t} \int_{B\left(x_{0}, 2\right) \backslash B\left(x_{0}, 1\right)}(p|\nabla u|+|\nabla p|) \\
& \leqslant C\left(\int_{0}^{t} \int_{B\left(x_{0}, 2\right) \backslash B\left(x_{0}, 1\right)}\left(|\nabla u|^{2}+1\right)\right)^{1 / 2} \cdot\left(\int_{0}^{t} \int_{B\left(x_{0}, 2\right) \backslash B\left(x_{0}, 1\right)}\left(|\nabla p|^{2}+p^{2}\right)\right)^{1 / 2} \\
& \leqslant C\left(1+\|h\|_{L^{\infty}(\Omega)}\right) \sqrt{t}\left(\int_{0}^{t} \int_{B\left(x_{0}, 2\right) \backslash B\left(x_{0}, 1\right)}\left(|\nabla p|^{2}+p^{2}\right)\right)^{1 / 2} .
\end{aligned}
$$

Note that in the last step of the above inequality, we used the Caccioppoli-type inequality:

$$
\begin{aligned}
\int_{B\left(x_{0}, 2\right)}|\nabla u|^{2} & \leqslant C \int_{B\left(x_{0}, 4\right)}\left(u^{2}+h^{2}\right) \leqslant C(n, \kappa)\left(1+\|h\|_{L^{2}(\Omega)}\right)^{2} \\
& \leqslant C(n, \kappa)\left(1+\|h\|_{L^{\infty}(\Omega)}\right)^{2}
\end{aligned}
$$

which can be proved by multiplying Equation (2.35) on both sides by $\tilde{\chi}^{2} u$ and doing integration by parts, for some cutoff function $\tilde{\chi}$. By inequality (2.32), the last term in (2.43) can be controlled by $C(\kappa, \beta) t^{\beta}$ for any positive number $\beta$. For the simplicity of later calculation, we choose $\beta=\frac{3}{4}$. Note that $\|h\|_{L^{\infty}(\Omega)}<$ $\left|h\left(x_{0}\right)\right|+[h]_{C^{1 / 2}(\Omega)}$. Let $L=1+\left|h\left(x_{0}\right)\right|+[h]_{C^{1 / 2}(\Omega)}$, then we have

$$
\begin{aligned}
|F(t)| & \leqslant\left|\int_{0}^{t} \int_{X} w \chi h p\right|+\left.\left|\int_{0}^{t} \int_{B\left(x_{0}, 2\right) \backslash B\left(x_{0}, 1\right)} u^{2}\right| \nabla \chi\right|^{2} p \mid+C L t^{5 / 4} \\
& \leqslant\left|\int_{0}^{t} \int_{X} w \chi h p\right|+4 \int_{0}^{t} \int_{B\left(x_{0}, 2\right) \backslash B\left(x_{0}, 1\right)} p+C L t^{5 / 4} .
\end{aligned}
$$

The second term on the right hand side of the above inequality can be absorbed in the last term, due to the exponential decay of $p$ and Euclidean volume growth condition (see Propositions 2.20 and 2.3). On the other hand, since $p w$ has zero integral, we have

$$
\begin{aligned}
\left|\int_{0}^{t} \int_{X} w \chi h p\right| & =\left|\int_{0}^{t} \int_{X}\left(\chi h-h\left(x_{0}\right)\right) p w\right| \\
& \leqslant\left|\int_{0}^{t} \int_{B\left(x_{0}, 2\right)}\left(\chi h-h\left(x_{0}\right)\right) p w\right|+\left|\int_{0}^{t} \int_{X \backslash B\left(x_{0}, 2\right)} h\left(x_{0}\right) p w\right| .
\end{aligned}
$$

However, as $\chi h-h\left(x_{0}\right)$ vanishes at $x_{0}$, we have

$$
\left|\chi h-h\left(x_{0}\right)\right| \leqslant[h]_{C^{1 / 2}(\Omega)} \cdot d^{1 / 2}\left(x, x_{0}\right)
$$


Consequently, we have

$$
\begin{aligned}
\left|\int_{0}^{t} \int_{B\left(x_{0}, 2\right)}\left(\chi h-h\left(x_{0}\right)\right) p w d \mu_{x} d s\right| & <C[h]_{C^{1 / 2}(\Omega)} \int_{0}^{t} s^{1 / 4} d s<C L t^{5 / 4}, \\
\left|\int_{0}^{t} \int_{X \backslash B\left(x_{0}, 2\right)} h\left(x_{0}\right) p w d \mu_{x} d s\right| & <2\left|h\left(x_{0}\right)\right| \int_{0}^{t} \int_{X \backslash B\left(x_{0}, 2\right)} p d \mu_{x} d s \\
& <C L t^{5 / 4} .
\end{aligned}
$$

Plugging the above inequalities into (2.44), we obtain

$$
|F(t)|<C L t^{5 / 4}, \quad \int_{0}^{1} \frac{|F(s)|}{s^{2}} d s<C L \int_{0}^{1} s^{-3 / 4} d s<C L .
$$

Recall that $L=1+\left|h\left(x_{0}\right)\right|+[h]_{C^{1 / 2}(\Omega)}$. Plugging the above inequalities into (2.41), we obtain the desired estimate of $\left|\nabla u\left(x_{0}\right)\right|$.

Combining Proposition 2.24 and the $C^{\alpha}$-estimate (see [38, Theorem 4.1]) for bounded heat solutions, one can derive the gradient estimate for heat solutions. Alternatively, for bounded heat solution $u$, one can follow the proof of Lemma A.4 to obtain the uniform bound of $|\nabla u|$ by De-Giorgi iteration process. Another interesting application of Cheng-Yau type inequality is the following Liouville theorem.

COROLlary 2.25 (Liouville theorem). Suppose $u$ is a harmonic function on $\mathcal{R}$ with sublinear growth condition, that is,

$$
\limsup _{r \rightarrow \infty} r^{-1}\|u\|_{L^{\infty}(B(x, r) \cap \mathcal{R})}=0
$$

for every $x \in X$, then $u \equiv C$. In particular, every bounded harmonic function on $\mathcal{R}$ is a constant.

Proof. By virtue of Proposition 2.19, the extension property of subharmonic functions, we know that $u \in N_{\text {loc }}^{1,2}(X)$. In light of Proposition 2.11, the density property of smooth functions in $N_{0}^{1,2}(\Omega)$, it is clear that $\Delta u=0$ on $X$ in the weak sense. Fix $x_{0} \in \mathcal{R}$ and a large $r>0$, by Cheng-Yau estimate in Proposition 2.24, we have

$$
|\nabla u|\left(x_{0}\right)<C \frac{\|u\|_{L^{\infty}\left(B\left(x_{0}, r\right)\right)}}{r}=C \frac{\|u\|_{L^{\infty}\left(B\left(x_{0}, r\right) \cap \mathcal{R}\right)}}{r} .
$$

Let $r \rightarrow \infty$, the sublinear condition (2.46) implies that $|\nabla u|\left(x_{0}\right)=0$. It follows that $\nabla u \equiv 0$ on $\mathcal{R}$ by the arbitrary choice of $x_{0} \in \mathcal{R}$. Consequently, $u \equiv C$ on $\mathcal{R}$. 
Proposition 2.26 (Estimates for Dirichlet problem solution). Suppose $\Omega$ is a bounded open set of $X, f$ is a continuous function in $N^{1,2}(\Omega)$. Then we have the following properties.

- There is a unique solution $u \in N^{1,2}(\Omega)$ solving the Dirichlet problem

$$
\Delta u=0, \quad \text { in } \Omega ;\left.\quad(u-f)\right|_{\partial \Omega}=0
$$

in the weak sense of traces. In other words, $\Delta u=0$ in the weak sense and $u-f \in N_{0}^{1,2}(\Omega)$.

- Weak maximum principle holds for $u$, that is,

$$
\sup _{x \in \Omega} u(x)=\sup _{x \in \partial \Omega} u(x), \quad \inf _{x \in \Omega} u(x)=\inf _{x \in \partial \Omega} u(x) .
$$

- Strong maximum principle holds for $u$, that is, if there is an interior point $x_{0} \in$ $\Omega$ such that $u\left(x_{0}\right)=\sup _{x \in \Omega} u(x)$ or $u\left(x_{0}\right)=\inf _{x \in \Omega} u(x)$, then $u$ is a constant.

Proof. The existence and uniqueness of the Dirichlet problem follows from Theorems 7.12 and 7.14 of Cheeger's work [5], where a much more general case was considered. The weak maximum principle follows from the uniqueness. Also, the weak maximum principle was proved by Shanmugalingam in [36]. The strong maximum principle follows from elliptic Harnack estimates, which is a consequence of the volume doubling and $(1,2)$-Poincare inequality. This is due to the work of Sturm in [42]. The manifold case was obtained by Grigor'yan in [27], and Saloff-Coste in [38]. More information can be found in the beautiful survey [39] by Saloff-Coste.

We write down an elementary proof here for the convenience of the readers, based on the excellent underlying geometry. Here we follow [30, Corollary 6.4]. Without loss of generality, we assume $u \geqslant 0$ on $\partial \Omega$ and $u$ is not a constant. It suffices to show that $u>0$ in $\Omega$. Clearly, by classical harmonic function theory on Riemannian manifold and continuity of $u$ (see Proposition 2.29), it is clear that $u>0$ on $\Omega \cap \mathcal{R}$. Therefore, we only need to show that $u>0$ on $\Omega \cap \mathcal{S}$. We argue by contradiction. If this statement were wrong, we can find a point $y_{0} \in \Omega \cap \mathcal{S}$ such that $u\left(y_{0}\right)=0$. Choose $r$ small enough such that $B\left(y_{0}, 2 r\right) \subset \Omega$. For each small $\epsilon$, choose $\tau$ small enough such that

$$
\left|\Omega_{\tau} \cap B\left(y_{0}, 2 r\right)\right|<\epsilon\left|B\left(y_{0}, 2 r\right)\right|,
$$

where $\Omega_{\tau}=\{x \in \Omega \mid u(x) \leqslant \tau\}$. Note that $\tau$ can be chosen since $\Omega_{0} \cap B\left(y_{0}, 2 r\right)$ is a subset of $\mathcal{S}$ which has zero measure, and $u$ is continuous. Now consider the function $\tau-u$, which is obviously harmonic. Let $(\tau-u)^{+}$be $\max \{\tau-u, 0\}$. Then 
$(\tau-u)^{+}$is a bounded, continuous, subharmonic function in $N^{1,2}\left(B\left(y_{0}, 2 r\right)\right)$. So Moser (or Nash-Moser-De-Giorgi) iteration applies to obtain

$$
\sup _{B\left(y_{0}, r\right)}\left|(\tau-u)^{+}\right|^{2} \leqslant C \int_{B\left(y_{0}, 2 r\right)}\left|(\tau-u)^{+}\right|^{2}=C \int_{\Omega_{\tau} \cap B\left(y_{0}, 2 r\right)}\left|(\tau-u)^{+}\right|^{2} \leqslant C \epsilon r^{2 n} \tau^{2}
$$

for some $C=C(n, \kappa)$. Choose $\epsilon$ small enough such that $C \epsilon^{2} r^{2 n}<\frac{1}{4}$. Then we have

$$
\sup _{B\left(y_{0}, r\right)}\left|(\tau-u)^{+}\right|<\frac{\tau}{2},
$$

which implies that $u>\tau / 2$ on $B\left(y_{0}, r\right)$. In particular, $u\left(y_{0}\right) \geqslant \tau / 2>0$, which contradicts the assumption $u\left(y_{0}\right)=0$.

Clearly, the essential ingredient in the proof of the strong maximum principle of Proposition 2.26 is a delicate use of elliptic Moser iteration. In Equation (2.47), if we replace the operator $\Delta$ by $\square$, the heat operator, then one can easily obtain a strong maximum principle for heat equation solutions, based on a parabolic Moser iteration. The details are left to the interested readers. On the other hand, if we replace the right hand side of Equation (2.47) by a function $h$, we can also obtain uniqueness and existence of solutions.

PROPOSITION 2.27 (Existence and uniqueness of Poisson equation solution). Suppose $\Omega$ is a bounded open set of $X, f \in N^{1,2}(\Omega), h \in L^{2}(\Omega)$. Then there exists a unique $u \in N^{1,2}(\Omega)$ such that

$$
\Delta u=h, \quad \text { in } \Omega ;\left.\quad(u-f)\right|_{\partial \Omega}=0 .
$$

Proof. First, let us consider the Poisson equation

$$
\Delta v=h, \quad \text { in } \Omega ; \quad v \in N_{0}^{1,2}(\Omega) .
$$

By standard functional analysis, the existence of the above equation is guaranteed by Riesz representation theorem. The uniqueness follows from the irreducibility of $\mathscr{E}$. Second, it is obvious that there is a bijective map between the solution $u$ of (2.49) and harmonic solution $w$ of (2.47) by $u=w+v$. Therefore, the existence and uniqueness of (2.49) follows from Proposition 2.26.

Combining the strong maximum principle for harmonic functions in Proposition 2.26 with the heat kernel estimates, we obtain the strong maximum principle for subharmonic functions. 
Proposition 2.28 (Strong maximum principle for subharmonic functions). Suppose $\Omega$ is a bounded domain of $X, u$ is a continuous subharmonic function in $N^{1,2}(\Omega)$. Then we have

$$
\sup _{x \in \Omega} u(x)=\sup _{x \in \partial \Omega} u(x) .
$$

In other words, the weak maximum principle holds for subharmonic functions. Moreover, if $\sup _{x \in \Omega} u(x)$ is achieved at some point $x_{0} \in \Omega$, then $u$ is a constant. Namely, the strong maximum principle holds for subharmonic functions.

Proof. The weak maximum principle is well known in the literature. For example, see [40, Lemma 4] and the reference therein.

The strong maximum principle can be proved the same as that in Proposition 2.26. Actually, $-w$ is a nonnegative superharmonic function on $\Omega$. If $w$ is not a constant, then we can regard $-w$ as $u$ in the second part of the proof of Proposition 2.26. Then everything goes through since only Moser iteration for subharmonic function is used there.

PROPOSITION 2.29 (Removing singularity of harmonic functions). Suppose $\Omega$ is an open domain in $X, u$ is a bounded harmonic function on $\Omega \backslash \mathcal{S}$. Then $u$ can be regarded as a harmonic function on $\Omega$. Moreover, on each compact subset of $\Omega$, $u$ is uniformly Lipschitz continuous. In particular, u can be extended continuously over the singular set $\Omega \cap \mathcal{S}$.

Proof. In light of Proposition 2.19, we see that $u \in N_{\text {loc }}^{1,2}(\Omega)$. Since $\Delta u=0$ on $\Omega \backslash \mathcal{S}$, we see that

$$
\int_{\Omega}\langle\nabla u, \nabla \varphi\rangle=0
$$

for every smooth test function supported on $\Omega \backslash \mathcal{S}$. However, such functions are dense in $N_{0}^{1,2}(\Omega)$, so the above equation actually holds from every $\varphi \in N_{c}^{1,2}(\Omega)$. Therefore, $u$ is harmonic in $\Omega$ by definition.

The Lipschitz continuity follows from Proposition 2.24 and the density and weak convexity of $\mathcal{R}$.

Note that the weak convexity of $\mathcal{R}$ is important in order that $u$ can be extended over singularities. For otherwise, the limit of $u\left(x_{i}\right)$ for $x_{i} \rightarrow x_{0}$ may depends on the choice of sequence $\left\{x_{i}\right\}$, where $x_{0} \in \mathcal{S}, x_{i} \in \mathcal{R}$. If $\mathcal{R}$ is not convex, there is an easy counterexample of Proposition 2.29. Let $X$ be the union of two cones $C\left(S^{3} / \Gamma\right)$ by identifying two vertices. Here $\Gamma$ is a finite subgroup of the isometry group of the standard $S^{3}$. In this case, $\mathcal{S}$ is the isolated vertex $O$. Let $u$ be 1 on one branch and 0 on the other, then it is clear that $u$ is a harmonic function on $X \backslash \mathcal{S}$. 
However, $u$ cannot take a value at $O$ so that $u$ is continuous. Of course, convexity of $\mathcal{R}$ is only a sufficient geometric condition to guarantee the continuity extension. It can be replaced by other weaker conditions. For example, a uniform bound of Poincaré constant, which is an analytic condition, will be enough to guarantee the continuous extension of harmonic functions (see Sturm [42, Corollary 3.3]). Moreover, based on Proposition 2.24, one can obtain uniform gradient estimate of $\left|\nabla p\left(t, \cdot, x_{0}\right)\right|$, which depends only on $t, n$ and $\kappa$. Hence the heat kernel $p\left(t, \cdot, x_{0}\right)$ is a continuous function on $X \times(0, \infty)$. Therefore, by approximation, the estimate in Proposition 2.20 holds on every point on $X$, even if this point is singular.

\subsection{Approximation functions of distance.}

PROPOSITION 2.30 (Almost superharmonicity of distance function). Suppose $x_{0} \in X, r(x)=d\left(x, x_{0}\right)$. Then we have

$$
\Delta r^{2} \leqslant 4 n
$$

in the weak sense. In other words, for every nonnegative $\chi \in N_{c}^{1,2}(X)$, we have

$$
-\int_{X}\left\langle\nabla r^{2}, \nabla \chi\right\rangle \leqslant \int_{X} 4 n \chi
$$

Proof. Let us first assume $x_{0} \in \mathcal{R}$. Clearly, away from the generalized cut locus $\mathcal{C}_{x}$, we have $\Delta r^{2} \leqslant 4 n$ in the classical sense. Therefore, $\Delta r^{2} \leqslant 4 n$ on $\mathcal{R}$ in the distribution sense, same as the smooth Riemannian manifold case. Since smooth cutoff functions supported on $\mathcal{R}$ are dense in $N_{0}^{1,2}(X)$ (see Corollary 2.12 and Proposition 2.11 , where $\operatorname{codim}(\mathcal{S})>2$ is essentially used), we see that for every $\chi \in N_{c}^{1,2}(X)$, inequality (2.52) holds true.

Now suppose $x_{0} \in \mathcal{S}$, we can choose regular points $x_{i} \rightarrow x_{0}$. Let $r_{i}=d\left(x_{i}, \cdot\right)$, then for each nonnegative function $\chi \in N_{c}^{1,2}(X)$, we have

$$
-\int_{X}\left\langle\nabla r_{i}^{2}, \nabla \chi\right\rangle \leqslant \int_{X} 4 n \chi
$$

Let $\Omega$ be a bounded open set containing the support of $\chi$. Then $r_{i}^{2}$ weakly converges to a unique limit in $N^{1,2}(\Omega), r_{i}^{2}$ strongly converges to $r^{2}$ in $L^{2}(\Omega)$. This means that $r^{2}$ is the weak limit of $r_{i}^{2}$ in $N^{1,2}(\Omega)$. It follows that

$$
-\int_{X}\left\langle\nabla r^{2}, \nabla \chi\right\rangle=-\lim _{i \rightarrow \infty} \int_{X}\left\langle\nabla r_{i}^{2}, \nabla \chi\right\rangle \leqslant \int_{X} 4 n \chi .
$$

In view of Proposition 2.30, we can obtain many rigidity theorems. 
LEMMA 2.31 (Cheeger-Gromoll type splitting). Suppose $X$ contains a straight line $\gamma$. Then there is a length space $N$ such that $X$ is isometric to $N \times \mathbb{R}$ as metric spaces.

Proof. The proof is almost the same as the classical one. However, we shall take this as an opportunity to check the analysis tools developed in previous subsections. Actually, fix $x_{0} \in \gamma$, we can divide $\gamma$ into two rays $\gamma^{+}$and $\gamma^{-}$. Accordingly, there are Busemann functions $b^{+}$and $b^{-}$. Proposition 2.30 implies that $\Delta r \leqslant(2 n-1) / r$ in the weak sense, which in turn forces both $b^{+}$and $b^{-}$to be subharmonic functions. By triangle inequality, we know $b^{+}+b^{-} \geqslant 0$ globally and achieve 0 on $x_{0}$. It follows from strong maximum principle, by Proposition 2.28, that $b^{+}+b^{-} \equiv 0$. Consequently, $b^{+}$is harmonic. Then Weitzenböck formula implies that in the weak sense, we have

$$
0=\frac{1}{2} \Delta\left|\nabla b^{+}\right|^{2}=\left|\operatorname{Hess}_{b^{+}}\right|^{2} .
$$

Since $b^{+}$is harmonic, it is harmonic on $\mathcal{R}=X \backslash \mathcal{S}$. By standard improving regularity theory of harmonic functions on smooth manifold, we see that $b^{+}$is a smooth function on $\mathcal{R}$ satisfying

$$
\left|\nabla b^{+}\right|^{2} \equiv 1, \quad\left|\operatorname{Hess}_{b^{+}}\right|^{2} \equiv 0 .
$$

Up to this step, everything is the same as the classical case. However, since the regular part $\mathcal{R}$ is not complete, the following argument is slightly different. On $\mathcal{R}$, since $\mathcal{L}_{\nabla b^{+}} g=2 \mathrm{Hess}_{b^{+}}=0$, we see that $\nabla b^{+}$is a Killing field. The flow generated by $\nabla b^{+}$preserves metrics, and in particular the volume element. By the high codimension of $\mathcal{S}$, weak convexity of $\mathcal{R}$ and the essential gap of volume density between regular and singular points, one can obtain that the flow generated by $\nabla b^{+}$preserves regularity. The full details will be explained as follows.

Let $\varphi_{t}$ be the time $t$ flow map generated by $\nabla b^{+}$when it is well defined. In other words, we have $(d / d t) \varphi_{t}(x)=\left.\nabla b^{+}\right|_{\varphi_{t}(x)}$ whenever $\varphi_{t}(x) \in \mathcal{R}$.

Claim 2.32 (Existence of flows away from small sets). For each fixed $x_{0} \in X$ and $A>0$, there is a set $E_{A}$ such that $\varphi_{t}(x)$ exists and locates in $\mathcal{R}$ for all $x \in B\left(x_{0}, A\right) \backslash E_{A}$ and $t \in[-A, A]$. Moreover, we have

$$
\operatorname{dim}_{\mathcal{M}} E_{A} \leqslant \operatorname{dim}_{\mathcal{M}} \mathcal{S}+1<2 n-2 .
$$

Consequently, there is a measure-zero set $E$ such that $\varphi_{t}(x)$ exists and locates in $\mathcal{R}$ for all $x \in X \backslash E$ and $t \in(-\infty, \infty)$.

Fix $x_{0} \in X$ and choose $\xi$ to be a very small positive number, $A$ to be a large positive number. Let $q_{0}$ be the Minkowski codimension of $\mathcal{S}$, that is, 
$q_{0}=2 n-\operatorname{dim}_{\mathcal{M}} \mathcal{S}, \epsilon$ be a very small number to be used in the volume estimate related to Minkowski codimension. Note that $d(\cdot, \mathcal{S})$ is a Lipschitz function with Lipschitz constant 1 . By perturbing $d(\cdot, \mathcal{S})$, we can find a smooth hypersurface $\Sigma_{\xi}$ (see Corollary B.3 for more details) such that

$$
\begin{aligned}
\mid B\left(x_{0}, 3 A\right) & \left.\cap \Sigma_{\xi}\right|_{\mathcal{H}^{2 n-1}} \\
\frac{1}{H} \xi & <d(x, \mathcal{S})<H \xi, \quad \forall x \in \xi_{\xi} \cap B\left(x_{0}, 2 A\right) .
\end{aligned}
$$

Note that the $\xi^{q_{0}-\epsilon}$ in the first inequality comes from the fact that

$$
\operatorname{dim}_{\mathcal{M}} \mathcal{S}=2 n-q_{0}
$$

and the application of coarea formula. The constant $C$ in the first inequality depends both on $\epsilon$ and the set $B\left(x_{0}, 3 A\right)$. The constant $H$ in the second inequality depends on $\kappa, n$ and comes from the perturbation technique. $H$ will be fixed in the following discussion. $C$ may vary from line to line, as usual. Basically, $\Sigma_{\xi}$ is a level set of a smooth function $\tilde{f}$ comparable to $d(\cdot, \mathcal{S})$. Hence $\Sigma_{\xi}$ is the boundary of $D_{\xi}=\{x \mid \tilde{f}(x) \leqslant \xi\}$ and

$$
B\left(\mathcal{S}, \frac{\xi}{H}\right) \subset D_{\xi} \subset B(\mathcal{S}, H \xi)
$$

Define a set

$$
E_{A, \xi} \triangleq\left\{x \in \overline{B\left(x_{0}, A\right)} \mid d\left(\varphi_{t}(x), \mathcal{S}\right) \leqslant \frac{1}{H} \xi, \quad \text { for some } t \in[-A, A]\right\} .
$$

By (2.54), the inequality in the above equation means that $\varphi_{t}(x) \in D_{\xi}$. Now we decompose $E_{A, \xi}$ into two parts $I$ and $I I$ as follows.

$$
\begin{gathered}
I=\left\{x \in \overline{B\left(x_{0}, A\right)} \mid d(x, \mathcal{S}) \leqslant H \xi\right\} \cap E_{A, \xi}, \\
I I=\left\{x \in \overline{B\left(x_{0}, A\right)} \mid d(x, \mathcal{S})>H \xi\right\} \cap E_{A, \xi} .
\end{gathered}
$$

By the Minkowski dimension assumption of $\mathcal{S}$, we know that

$$
|I| \leqslant\left|\left\{x \in \overline{B\left(x_{0}, A\right)} \mid d(x, \mathcal{S}) \leqslant H \xi\right\}\right| \leqslant C \xi^{q_{0}-\epsilon},
$$

where $|\cdot|$ means the $2 n$-dimensional Hausdorff measure. Taking every point $y \in I I$, it follows from definition and (2.54) that $y \in\left(D_{\xi}\right)^{c}$. However, in light of the definition of $E_{A, \xi}$, one can flow it into $D_{\xi}$ for some $t \in[-A, A]$. This means that one can flow $y$ to a point on $\Sigma_{\xi}=\partial D_{\xi}$ at some time $t \in[-A, A]$. Since $\left|\nabla b^{+}\right|=1$, it is clear that $d\left(y, \varphi_{t}(y)\right) \leqslant|t|$. Then triangle inequality implies that

$$
d\left(x_{0}, \varphi_{t}(y)\right)<d\left(x_{0}, y\right)+d\left(y, \varphi_{t}(y)\right) \leqslant A+|t| \leqslant 2 A<3 A .
$$


Therefore, the set $I I$ can be locally regarded as a bundle over $\Sigma_{\xi} \cap B\left(x_{0}, 3 A\right)$. Note that along the flow line of the Killing field $\nabla b^{+}, \varphi_{t}$ preserves local isometry. We equip the set $\left\{\Sigma_{\xi} \cap B\left(x_{0}, 3 A\right)\right\} \times[-A, A]$ with the obvious product measure. Consider the map

$$
\begin{aligned}
\varphi: \Omega \subset\left\{\Sigma_{\xi} \cap B\left(x_{0}, 3 A\right)\right\} \times[-A, A] & \mapsto X, \\
(x, t) & \mapsto \varphi_{t}(x) .
\end{aligned}
$$

Here $\Omega$ is the maximal subset of $\left\{\Sigma_{\xi} \cap B\left(x_{0}, 3 A\right)\right\} \times[-A, A]$ such that $\varphi(x, t)=\varphi_{t}(x)$ is well defined. It is clear that $\varphi$ decrease volume whenever the flow line is not perpendicular to $\Sigma_{\xi}$. It follows that

$$
|I I| \leqslant|\Omega| \leqslant\left|\left\{\Sigma_{\xi} \cap B\left(x_{0}, 3 A\right)\right\}\right|_{\mathcal{H}^{2 n-1}} \cdot 3 A \leqslant C A \xi^{q_{0}-\epsilon-1} .
$$

Combining the above inequality with (2.56), we have

$$
\left|E_{A, \xi}\right| \leqslant|I|+|I I| \leqslant C \xi^{q_{0}-\epsilon}+C A \xi^{q_{0}-\epsilon-1} \leqslant C \xi^{q_{0}-\epsilon-1},
$$

where the last $C$ depends on $n, \kappa$ and $B\left(x_{0}, 3 A\right)$.

We observe that

$$
\left\{x \mid d\left(x, E_{A, \xi}\right)<H^{-1} \xi\right\} \subset E_{2 A, 2 \xi} .
$$

In fact, if $x$ locates in the $H^{-1} \xi$-neighborhood of $E_{A, \xi}$, then we can find a point $y \in E_{A, \xi}$ such that $d\left(x, E_{A, \xi}\right)=d(x, y)=\delta<H^{-1} \xi$. So we can find a shortest geodesic connecting $x$ to $y$ satisfying $\gamma(0)=x$ and $\gamma(\delta)=y$, with $|\gamma|=\delta$. Note that we can assume $\gamma$ is a smooth geodesic. For otherwise, we have

$$
d(x, \mathcal{S}) \leqslant \delta<H^{-1} \xi,
$$

which automatically implies $x \in E_{2 A, 2 \xi}$ by the definition in (2.55). For the same reason, triangle inequality allows us to assume

$$
d(\gamma, \mathcal{S})>H^{-1} \xi
$$

As $y \in E_{A, \xi}$, following its definition in (2.55), we can find a $t_{0} \in[-A, A]$ such that

$$
d\left(\varphi_{t_{0}}(y), \mathcal{S}\right) \leqslant H^{-1} \xi .
$$

Let $s_{0}$ be the smallest positive value such that $d\left(\varphi_{s_{0}}(\gamma), \mathcal{S}\right) \leqslant H^{-1} \xi$. The combination of (2.59) and (2.60) then yields that $0<\left|s_{0}\right| \leqslant\left|t_{0}\right|$. Note that $\varphi_{s}(\gamma)$ is well defined on $\left(-\left|s_{0}\right|,\left|s_{0}\right|\right)$. So the length of $\varphi_{s}(\gamma)$ is the same as the length of 
$\gamma$ for each $s \in\left(-\left|s_{0}\right|,\left|s_{0}\right|\right)$. Now we are ready to estimate the distance between $\varphi_{s_{0}}(x)$ and the singular set $\mathcal{S}$ :

$$
\begin{aligned}
d\left(\varphi_{s_{0}}(x), \mathcal{S}\right) & \leqslant\left|\varphi_{s_{0}}(\gamma)\right|+d\left(\varphi_{s_{0}}(\gamma), \mathcal{S}\right)=|\gamma|+d\left(\varphi_{s_{0}}(\gamma), \mathcal{S}\right) \\
& <H^{-1} \xi+H^{-1} \xi=\frac{2 \xi}{H} .
\end{aligned}
$$

Note that $E_{A, \xi} \subset \overline{B\left(x_{0}, A\right)}$. Triangle inequality implies that $x \in \overline{B\left(x_{0}, 2 A\right)}$. Recall also that $s_{0} \in[-A, A] \subset[-2 A, 2 A]$. In light of definition equation (2.55), the above inequality implies that $x \in E_{2 A, 2 \xi}$. So we finish the proof of (2.58).

Let $\xi=\xi_{i} \rightarrow 0$ and define

$$
E_{A} \triangleq \bigcap_{i=1}^{\infty} E_{A, \xi_{i}} .
$$

We obtain a set $E_{A} \subset \overline{B\left(x_{0}, A\right)}$ such that $\varphi_{t}(x) \in \mathcal{R}$ for each point $x \in$ $\overline{B\left(x_{0}, A\right)} \backslash E_{A}$ and $t \in[-A, A]$. Furthermore, from definition equation (2.61), it is clear that $E_{A} \subset E_{A, \xi}$, which together with (2.58) implies that the $H^{-1} \xi$ neighborhood of $E_{A}$ is contained in $E_{2 A, 2 \xi}$. Consequently, it follows from (2.57) (replacing $A$ by $2 A, \xi$ by $2 \xi$ ) that

$$
\left|\left\{x \mid d\left(x, E_{A}\right)<H^{-1} \xi\right\}\right|_{\mathcal{H}^{2 n}} \leqslant C \xi^{q_{0}-\epsilon-1}=C\left(H^{-1} \xi\right)^{q_{0}-\epsilon-1},
$$

where the last $C$ depends on $n, \kappa, H, \epsilon$ and the set $B\left(x_{0}, 3 A\right)$, is independent of the choice of $\xi$. Since $\epsilon$ can be any small number, the above inequality implies (2.53) by the definition of Minkowski dimension (see Definition 2.2).

Then we set $A=A_{i} \rightarrow \infty$ and define $E \triangleq \bigcup_{i=1}^{\infty} E_{A_{i}}$. As a union of countably many measure-zero sets, $E$ is clearly a measure-zero set. Clearly, for each $x \in X \backslash E$, and each $t \in(-\infty, \infty)$, we can always find a large $A_{i}$ such that $x \in B\left(x_{0}, A_{i}\right)$ and $t \in\left(-A_{i}, A_{i}\right)$. Then it follows that $\varphi_{t}(x)$ exists and locates in $B\left(x_{0}, 2 A_{i}\right) \cap \mathcal{R} \subset \mathcal{R}$. So we finish the proof of Claim 2.32.

CLAIM 2.33 (Flow lines preserve regularity). Suppose $x_{0}$ is a regular point, then the whole flow line of $\nabla b^{+}$initiated from $x_{0}$ is defined for all time and stays in $\mathcal{R}$.

Suppose Claim 2.33 was wrong. Without loss of generality, we can assume that the flow image of $x_{0}$ hits singularity the first time at $T_{0}>0$. Let $\varphi_{t}$ be the time $t$ flow map generated by $\nabla b^{+}$. Fix $\epsilon$ very small, then $y_{0}=\varphi_{T_{0}-\epsilon}\left(x_{0}\right)$ is a regular point. By Claim 2.32, we see that $\varphi_{T_{0}-\epsilon}$ is well defined away from a measure-zero 
set $E$. Furthermore, it preserves volume element and length element. For each $r \in(0,1)$, we define $\chi_{r}$ as

$$
\chi_{r}(x)= \begin{cases}r-d\left(x, x_{0}\right) & \text { if } d\left(x, x_{0}\right)<r \\ 0 & \text { if } d\left(x, x_{0}\right) \geqslant r\end{cases}
$$

The function $f_{r, \epsilon}=\chi_{r} \circ \varphi_{T_{0}-\epsilon}^{-1}$ is defined on $X \backslash E$. Actually, by choosing $A \gg T_{0}+1$, we know that $f_{r, \epsilon}$ is defined on $B\left(y_{0}, A\right) \backslash E_{A}$ and vanishes outside $B\left(y_{0}, 0.5 A\right)$. This is a simple application of triangle inequality. If $x \in B\left(y_{0}\right.$, $A) \backslash E_{A}$ and $d\left(x, y_{0}\right)>0.5 A>5\left(T_{0}+1\right)$, then we have

$$
\begin{aligned}
d\left(\varphi_{T_{0}-\epsilon}^{-1}(x), x_{0}\right) & \geqslant-d\left(\varphi_{T_{0}-\epsilon}^{-1}(x), x\right)+d\left(x, x_{0}\right) \\
& \geqslant-d\left(\varphi_{T_{0}-\epsilon}^{-1}(x), x\right)-d\left(y_{0}, x_{0}\right)+d\left(x, y_{0}\right) \\
& \geqslant-2\left(T_{0}-\epsilon\right)+5\left(T_{0}+1\right)>3 T_{0}+5>r .
\end{aligned}
$$

Then $\chi_{r}\left(\varphi_{T_{0}-\epsilon}^{-1}(x)\right)=0$ by definition of $\chi_{r}$. By the local isometry property of $\varphi_{T_{0}-\epsilon}$, we obtain that $\left|\nabla\left(\chi_{r} \circ \varphi_{T_{0}-\epsilon}^{-1}\right)\right| \leqslant 1$ on $B\left(y_{0}, A\right) \backslash E_{A}$. Recall that $E_{A}$ has codimension at least 2 by (2.53). Then it is clear that $f_{r, \epsilon} \in N_{0}^{1,2}(X)$. Note that $f_{r, \epsilon}$ has a version $\tilde{f}_{r, \epsilon}$ which is globally Lipschitz with Lipschitz constant 1 . In other words, one can find a measure-zero set $F$ such that $\tilde{f}_{r, \epsilon}=f_{r, \epsilon}$ on $X \backslash F$. The function $\tilde{f}_{r, \epsilon}$ can be obtained as follows. Let $f_{r, \epsilon}(t)$ be the heat flow solution initiated from $f_{r, \epsilon} \in N^{1,2}(X)$. Then $\left|\nabla f_{r, \epsilon}(t)\right|$ is a heat subsolution. Note that here we used the condition $\operatorname{dim}_{\mathcal{M}} \mathcal{S}<2 n-3$ and the weak convexity of $\mathcal{R}$, to guarantee that $\left|\nabla f_{r, \epsilon}(t)\right| \in N_{\text {loc }}^{1,2}(X)$. Further details can be found in Appendix A. By maximum principle (on the space possibly has singularities), we see that $\left|\nabla f_{r, \epsilon}(t)\right| \leqslant 1$ for each $t>0$. Let $t_{i} \rightarrow 0$, then the limit of $f_{r, \epsilon}\left(t_{i}\right)$ can be chosen as $\tilde{f}_{r, \epsilon}$. Under the help of $\tilde{f}_{r, \epsilon}$, we shall see that $\varphi_{T_{0}-\epsilon}$ is an isometry from $X \backslash E$ to $X \backslash E$, by further adjusting $E$ with an extra measure-zero set if necessary. Actually, if $x \in \partial B\left(x_{0}, r\right) \backslash E$, then $y=\varphi_{T_{0}-\epsilon}(x)$ is a regular point. Note that $\tilde{f}_{r, \epsilon}\left(y_{0}\right)=r$ and $\tilde{f}_{r, \epsilon}(y)=0$. Since $\tilde{f}_{r, \epsilon}$ has uniform global Lipschitz constant 1 , we have $d\left(y, y_{0}\right) \geqslant r$. Therefore, $\varphi_{T_{0}-\epsilon}$ is a distance-expanding map from $X \backslash E$ to $X \backslash E$. By reversing the position of $x_{0}, y_{0}$ and using $-\nabla b^{+}$to generate flow, it is clear that $\varphi_{T_{0}-\epsilon}$ is distance-shrinking. Combining these two directions, we obtain

$$
\varphi_{T_{0}-\epsilon}^{-1}\left(B\left(y_{0}, r\right) \backslash E\right)=B\left(x_{0}, r\right) \backslash E .
$$

In particular, we see that

$$
\left|B\left(x_{0}, r\right)\right|=\left|B\left(y_{0}, r\right)\right|=\left|B\left(\varphi_{T_{0}-\epsilon}\left(x_{0}\right), r\right)\right| .
$$

Using triangle inequality and letting $\epsilon \rightarrow 0$, we have

$$
\left|B\left(x_{0}, r\right)\right|=\left|B\left(\varphi_{T_{0}}\left(x_{0}\right), r\right)\right|
$$


for each $r \in(0,1)$. However, $x_{0}$ is regular. For some $r \in(0,1)$, we have $\omega_{2 n}^{-1} r^{-2 n}\left|B\left(x_{0}, r\right)\right|>1-\left(\delta_{0} / 100\right)$. The same volume ratio estimate holds for the ball $B\left(\varphi_{T_{0}}\left(x_{0}\right), r\right)$. Therefore, $\varphi_{T_{0}}\left(x_{0}\right)$ is forced to be a regular point by Anderson's gap theorem (see Corollary 2.48). This contradicts the assumption of $T_{0}$. Therefore, the proof of Claim 2.33 is complete.

Let $N$ be the level set $b^{+}=0, N^{\prime}=N \cap \mathcal{R}$. Then it is clear that $\mathcal{R}=N^{\prime} \times \mathbb{R}$. Taking metric completion on both sides, we obtain $X=N \times \mathbb{R}$ as metric spaces.

Lemma 2.31 should be a special case of Gigli [25]. Its local version is the following lemma.

Lemma 2.34 (Metric cone rigidity). Suppose $x_{0} \in X, \Omega=B\left(x_{0}, 1\right)$. Then the following conditions are equivalent.

(1) Volume ratio same on scales 0.5 and 1 , that is, we have

$$
2^{2 n}\left|B\left(x_{0}, 0.5\right)\right|=\left|B\left(x_{0}, 1\right)\right| .
$$

(2) $\Omega$ is a volume cone, that is, for every $0<r_{1}<r_{2}<1$, we have

$$
r_{1}^{-2 n}\left|B\left(x_{0}, r_{1}\right)\right|=r_{2}^{-2 n}\left|B\left(x_{0}, r_{2}\right)\right| .
$$

(3) $r^{2} / 2$ is the unique weak solution of the Poisson equation

$$
\Delta u=2 n, \quad \text { in } \Omega ;\left.\quad\left(u-\frac{r^{2}}{2}\right)\right|_{\partial \Omega}=0 .
$$

(4) $r^{2} / 2$ induces local metric cone structure on $\Omega$. In other words, on $\Omega \backslash \mathcal{S}$, we have

$$
\operatorname{Hess}_{r^{2} / 2}-g \equiv 0
$$

Proof. (1) $\Rightarrow(2)$ : let $A(r)$ be the 'area' ratio function in Corollary 2.5, that is, $A(r)=\left|\partial B\left(x_{0}, r\right)\right| / r^{2 n-1}$. By Corollary 2.5, we know $A(r)$ is defined almost everywhere and is nonincreasing on its domain. Note that

$$
\frac{d}{d r}\left(\frac{\left|B\left(x_{0}, r\right)\right|}{r^{2 n}}\right)=\frac{\left|\partial B\left(x_{0}, r\right)\right|}{r^{2 n}}-\frac{2 n}{r} \frac{\left|B\left(x_{0}, r\right)\right|}{r^{2 n}}=\frac{2 n}{r}\left\{\frac{A(r)}{2 n}-\frac{\left|B\left(x_{0}, r\right)\right|}{r^{2 n}}\right\} \leqslant 0 .
$$

Combining (2.62) and (2.8), we have $(A(r) / 2 n)-\left(\left|B\left(x_{0}, r\right)\right| / r^{2 n}\right) \equiv 0$ for a.e. $r \in(0.5,1)$. In particular, we have

$$
\left|B\left(x_{0}, 1\right)\right|=\frac{A(1)}{2 n}=\int_{0}^{1} A(1) r^{2 n-1} d r
$$


where $A(1)$ is understood as $\lim _{r \rightarrow 1^{-}} A(r)$. On the other hand, it follows from (2.7) that

$$
\left|B\left(x_{0}, 1\right)\right|=\int_{0}^{1} A(r) r^{2 n-1} d r .
$$

So we have

$$
\int_{0}^{1}(A(r)-A(1)) r^{2 n-1} d r=0 .
$$

Note that $A(r)$ is a nonincreasing function. So the above equality means that

$$
A(1) \equiv A(r) \equiv \lim _{r \rightarrow 0^{+}} A(r) .
$$

It follows that $\left|B\left(x_{0}, r\right)\right|=(A(1) / 2 n) r^{2 n}$ for every $0<r<1$. In particular, $B\left(x_{0}, 1\right)$ is a volume cone.

(2) $\Rightarrow$ (3): suppose $u$ is the unique solution of the Poisson equation (2.64), we need to show that $u \equiv r^{2} / 2$. By uniqueness of weak solutions, it suffices to show that $\left(r^{2} / 2\right)-u$ is harmonic on $\Omega$, that is, for every $\varphi \in N_{c}^{1,2}(\Omega)$, we have $\int_{\Omega} \varphi \Delta\left(\left(r^{2} / 2\right)-u\right)=0$. By rescaling, we can also assume $0 \leqslant \varphi \leqslant 1$. Fix such a $\varphi$, we can choose $\epsilon$ small such that the support of $\varphi$ is contained in $B\left(x_{0}, 1-\epsilon\right)$. Define

$$
\eta(x)= \begin{cases}1 & \text { if } d\left(x, x_{0}\right)<1-\epsilon, \\ \frac{1-d\left(x, x_{0}\right)}{\epsilon} & \text { if } 1-\epsilon \leqslant d\left(x, x_{0}\right) \leqslant 1 .\end{cases}
$$

Note that $\eta \in N_{0}^{1,2}(\Omega),\left(r^{2} / 2\right)-u$ is superharmonic on $\Omega$. It follows from integration by parts that

$$
\int_{X} \eta \Delta\left(\frac{r^{2}}{2}-u\right)=-2 n \int_{\Omega} \eta+\frac{1}{\epsilon} \int_{B\left(x_{0}, 1\right) \backslash B\left(x_{0}, 1-\epsilon\right)} r \geqslant-O(\epsilon),
$$

where we used volume cone condition in the last step. Thus, we have

$$
\begin{aligned}
0 & \geqslant \int_{\Omega} \varphi \Delta\left(\frac{r^{2}}{2}-u\right)=\int_{\Omega} \eta \Delta\left(\frac{r^{2}}{2}-u\right)+\int_{\Omega}(\varphi-\eta) \Delta\left(\frac{r^{2}}{2}-u\right) \\
& \geqslant \int_{\Omega} \eta \Delta\left(\frac{r^{2}}{2}-u\right) \geqslant-O(\epsilon) .
\end{aligned}
$$

Let $\epsilon \rightarrow 0$, we obtain $\int_{\Omega} \varphi \Delta\left(\left(r^{2} / 2\right)-u\right)=0$. Consequently, $\left(r^{2} / 2\right)-u$ is harmonic by the arbitrary choice of $\varphi$.

$(3) \Rightarrow(4)$ : since $r^{2} / 2$ solves the Poisson equation with right hand side a constant, by standard bootstrapping argument for elliptic equation, we see that 
$r^{2} / 2$ is a smooth function on $\Omega \backslash \mathcal{S}$. Clearly, we have $\left|\nabla\left(r^{2} / 2\right)\right|^{2}=r^{2} / 2$. Taking Laplacian on both sides, Weitzenböck formula yields that $\left|\operatorname{Hess}_{r^{2} / 2}\right|^{2}=2 n$, which in turn implies that

$$
\left|\operatorname{Hess}_{r^{2} / 2}-g\right|^{2}=\left|\operatorname{Hess}_{r^{2} / 2}\right|^{2}-2 \Delta \frac{r^{2}}{2}+2 n=\left|\operatorname{Hess}_{r^{2} / 2}\right|^{2}-2 n=0 .
$$

Therefore, on $\Omega \backslash \mathcal{S}$, we have $\operatorname{Hess}_{r^{2} / 2}-g \equiv 0$ in the classical sense. Consequently, $\nabla\left(r^{2} / 2\right)$ is a conformal Killing field. Similar to the proof of Claim 2.33, one can show that the flow generated by $\nabla\left(r^{2} / 2\right)$ preserves regularity. Hence it is clear that $\Omega \backslash \mathcal{S}$ has a local metric cone structure, whose completion implies that $\bar{\Omega}$ is a unit ball in a metric cone.

(4) $\Rightarrow$ (1): for each $0<r<1$, note that $\left|B\left(x_{0}, r\right)\right|=\left|B\left(x_{0}, r\right) \backslash \mathcal{S}\right|$. Note the flow generated by $\nabla\left(r^{2} / 2\right)$ preserves regularity. More precisely, we have

$$
\mathcal{L}_{\nabla\left(r^{2} / 2\right)} g=g, \quad \mathcal{L}_{\nabla\left(r^{2} / 2\right)} d \mu=2 n d \mu .
$$

Then (2.63) follows from the integration of the above equation along flow lines.

Lemma 2.35 (Kähler cone splitting). Suppose $X \in \widetilde{\mathscr{K} \mathscr{S}}^{*}(n, \kappa)$ is a metric cone with vertex $x_{0}$. Then we can find a metric cone $C(Z)$ with vertex $z^{*}$ such that

$$
X=\mathbb{C}^{n-k} \times C(Z), \quad x_{0}=\left(0, z^{*}\right), \quad 2 \leqslant k \leqslant n .
$$

Moreover, there is no straight line in $C(Z)$ passing through $z^{*}$.

Proof. It suffices to show that if $X$ splits off a real straight line $\mathbb{R}$, then it splits off a complex line $\mathbb{C}$. In fact, if there is a straight line passing through $x_{0}$, we can find a function $h$ which is the Busemann function determined by the line. Therefore, $\nabla h$ is a parallel vector field with $|\nabla h| \equiv 1$. The Kähler condition implies that $J \nabla h$ is another parallel vector field satisfying $|J \nabla h| \equiv 1$ on $\mathcal{R}=X \backslash \mathcal{S}$. On the regular set, define function

$$
u=\left\langle J \nabla h, \nabla \frac{r^{2}}{2}\right\rangle,
$$

where $r$ is the distance to the vertex $x_{0}$. Metric cone condition implies that Hess $_{r^{2} / 2}=g$. Since $J \nabla h$ is parallel, we see that

$$
\nabla u=\operatorname{Hess}_{r^{2} / 2}(J \nabla h, \cdot)=J \nabla h .
$$

Recall that $\operatorname{Hess}_{h} \equiv 0$. Taking gradient of the above equation implies that $\operatorname{Hess}_{u} \equiv 0$. This forces that $\nabla u=J \nabla h$ is also a splitting direction. Note that 
although $u$ is only defined on $\mathcal{R}$, which is not complete, we can bypass this difficulty as done in the proof of Lemma 2.31, since $\nabla u$ is a Killing field preserving regularity. Therefore, we obtain a splitting factor $\mathbb{C}$. Since $J \nabla u=$ $-\nabla h$, the space spanned by $\nabla u$ and $J \nabla u$ is closed under the $J$-action. This induces the $J$-action closedness of the split linear space, which then must be $\mathbb{C}^{n-k}$ for some integer $k$. Because $X$ is not $\mathbb{C}^{n}$, we know the singular set is not empty, whose dimension restriction forces that $k \geqslant 2$.

For each rigidity property in Lemmas $2.31-2.35$, there should exist an 'almost' version. For example, Lemma 2.34 basically says that a volume cone implies a metric cone. Hence the 'almost' version is that for a unit geodesic ball $B\left(x_{0}, 1\right)$ whose volume ratio function $r^{-2 n}\left|B\left(x_{0}, r\right)\right|$ is very close to a constant function on $[0,1]$, then after proper rescaling, each ball $B\left(x_{0}, r\right)$ is very close to $B\left(x_{0}, 1\right)$ in the Gromov-Hausdorff topology. The basic idea is expressed clearly in [8]. We only interpret what they did. Actually, if volume ratio is almost a constant, then it is expected that $\left|\operatorname{Hess}_{r^{2} / 2}-g\right|$ has a small $L^{2}$-norm. However, since the regularity of distance function $r$ is bad, one should replace $r^{2} / 2$ by an approximation function, which is very close to $r^{2} / 2$ in $N^{1,2}$-norm on one hand, and has excellent regularity on the other hand. Such approximation function is nothing but the solution of the Poisson equation (2.64). For the purpose of developing 'almost' rigidity properties, one needs some technical preparation, which will be listed as Lemmas. Note that the space $\widetilde{\mathscr{K} \mathscr{S}}(n, \kappa)$ has scaling invariance. Therefore, we can always let the scale we are interested in to be 1 , to simplify the notations.

In view of Proposition 2.30, we can define many auxiliary radial functions, as in the classical case for Riemannian manifold (see [8]). For each $0<r<R<\infty$, define

$$
\begin{aligned}
\underline{U}(r) & \triangleq \frac{r^{2}}{4 n}, \quad \underline{G}(r) \triangleq \frac{r^{2-2 n}}{2 n(2 n-2) \omega_{2 n}}, \\
\underline{U}_{R} & \triangleq \frac{r^{2}-R^{2}}{4 n}, \quad \underline{G}_{R} \triangleq \frac{r^{2-2 n}-R^{2-2 n}}{2 n(2 n-2) \omega_{2 n}}, \\
\underline{L}_{R} & \triangleq \frac{r^{2-2 n} R^{2 n}-R^{2}}{2 n(2 n-2)}+\frac{r^{2}-R^{2}}{4 n} .
\end{aligned}
$$

Then by Proposition 2.30 and direct calculation, we have the following lemma.

LEMMA 2.36 (Existence of good radial comparison functions). Suppose $x_{0} \in X$. Let $r(x)=d\left(x, x_{0}\right)$ and define $\underline{U}_{1}(x)=\underline{U}_{1}(r(x)), \underline{G}_{1}=\underline{G}_{1}(r(x))$ and $\underline{L}_{1}(x)=$ $\underline{L}(r(x))$ as done in (2.67). Then we have

$$
\Delta \underline{U}_{1} \leqslant 1 \quad \text { on } X ;\left.\quad \underline{U}_{1}\right|_{\partial B\left(x_{0}, 1\right)}=0 .
$$




$$
\begin{aligned}
& \Delta \underline{G}_{1} \geqslant 0 \quad \text { on } B\left(x_{0}, 1\right) \backslash\left\{x_{0}\right\} ;\left.\quad \underline{G}_{1}\right|_{\partial B\left(x_{0}, 1\right)}=0 \text {. } \\
& \Delta \underline{L}_{1} \geqslant 1 \quad \text { on } B\left(x_{0}, 1\right) \backslash\left\{x_{0}\right\} ;\left.\quad \underline{L}_{1}\right|_{\partial B\left(x_{0}, 1\right)}=0 \text {. }
\end{aligned}
$$

Lemma 2.36 is used to improve the maximum principle. Same as that done by Abresch-Gromoll (see [1, Proposition 2.3]), we obtain the following estimate of excess function.

LEMMA 2.37 (Abresch-Gromoll type estimate). Suppose $x_{0} \in X, \gamma$ is a line segment centered at $x_{0}$ with length 2 , end points $p_{+}$and $p_{-}$. Let $e(x)$ be the excess function $d\left(x, p_{+}\right)+d\left(x, p_{-}\right)-2$. Then we have

$$
\sup _{x \in B\left(x_{0}, \epsilon\right)} e(x) \leqslant C \epsilon^{2 n /(2 n-1)}
$$

for each $\epsilon \in(0,1)$ and some universal constant $C=C(n)$.

Proof. Note that the excess function $e(x)$ around $x_{0}$ satisfies $\Delta e \leqslant 4(2 n-1)$ by Laplace comparison (see Proposition 2.30). The main ingredient of the proof is an application of maximum principle for subharmonic functions, and the existence of a poled function $\underline{L}$ such that $\Delta \underline{L} \geqslant 1$. In our case, both maximum principle (see Proposition 2.28) and the existence of $\underline{L}$ (see Lemma 2.36) are known. Therefore, the proof exactly follows the excess estimate of Abresch-Gromoll (see [1, Proposition 2.3]). Basically, for each $x \in B\left(x_{0}, \epsilon\right)$ and each number $c \in(0$, $\left.d\left(y, x_{0}\right)\right)$, one can apply maximum principle to obtain

$$
e(x)<\inf _{c \in\left(0, d\left(x, x_{0}\right)\right)}\left\{4(m-1) \underline{L}_{2 \epsilon}(c)+2 c\right\} \leqslant C \epsilon^{2 n /(2 n-1)},
$$

where we used the definition of $\underline{L}_{2 \epsilon}$ in (2.67).

Lemma 2.36 can also be applied to construct good cutoff functions.

Lemma 2.38 (Cutoff functions on annulus). Suppose $x_{0} \in X, 0<\rho<1<\infty$. Then there exists a function $\phi: X \rightarrow[0,1]$ such that

$$
\begin{aligned}
\phi & \in C^{\infty}\left(B\left(x_{0}, 1\right) \backslash \mathcal{S}\right), \quad \operatorname{supp} \phi \Subset B\left(x_{0}, 1\right), \quad \phi \equiv 1 \quad \text { on } B\left(x_{0}, \rho\right), \\
|\nabla \phi| & \leqslant c(n, \rho), \quad|\Delta \phi| \leqslant c(n, \rho), \quad \text { on } B\left(x_{0}, 1\right) \backslash \mathcal{S} .
\end{aligned}
$$

Furthermore, for each pair $\rho_{1}, \rho_{2}$ satisfying $0<\rho_{1}<\frac{1}{2}<2<\rho_{2}<\infty$, there exists a function $\phi: X \times[0,1]$ such that

$$
\begin{aligned}
\phi & \in C^{\infty}\left(\left(B\left(x_{0}, \rho_{2}\right) \backslash \overline{B\left(x_{0}, \rho_{1}\right)}\right) \cap \mathcal{R}\right), \quad \operatorname{supp} \phi \Subset B\left(x_{0}, \rho_{2}\right) \backslash \overline{B\left(x_{0}, \rho_{1}\right)}, \\
\phi & \equiv 1 \quad \text { on } B\left(x_{0}, \frac{\rho_{2}}{2}\right) \backslash \overline{B\left(x_{0}, 2 \rho_{1}\right)}, \\
|\nabla \phi| & \leqslant c\left(n, \rho_{1}, \rho_{2}\right), \quad|\Delta \phi| \leqslant c\left(n, \rho_{1}, \rho_{2}\right), \quad \text { on }\left(B\left(x_{0}, \rho_{2}\right) \backslash \overline{B\left(x_{0}, \rho_{1}\right)}\right) \cap \mathcal{R} .
\end{aligned}
$$


The proof of Lemma 2.38 is based on the maximum principle, solvability of Poisson equation and the fact that $\Delta \underline{L}_{R^{\prime}} \geqslant 1$ and $\Delta \underline{U}_{R^{\prime}} \leqslant 1$ for each $R^{\prime}>0$. With these properties, one can compare $\underline{L}_{1}$ with the Poisson equation solution $f$ which has same boundary value as $\underline{L}_{1}$. Then we construct a cutoff function based on the value of $f$. Since the proof follows that of [8] verbatim, we omit the details here.

LEMMA 2.39 (Harmonic approximation of local Busemann function). There exists a constant $c=c(n)$ with the following properties.

Suppose $x_{0} \in X, \gamma$ is a line segment centered at $x_{0}$ with length 2 , end points $p_{+}$and $p_{-}, \epsilon$ is an arbitrary small positive number, say $0<\epsilon<0.1$. In the ball $B\left(x_{0}, 4 \epsilon\right)$, define local Busemann functions

$$
b_{+}(x)=d\left(x, p_{+}\right)-d\left(x_{0}, p_{+}\right), \quad b_{-}(x)=d\left(x, p_{-}\right)-d\left(x_{0}, p_{-}\right) .
$$

Let $u_{ \pm}$be the harmonic functions in $B\left(x_{0}, 4 \epsilon\right)$ such that $\left.\left(u_{ \pm}-b_{ \pm}\right)\right|_{\partial B\left(x_{0}, 4 \epsilon\right)}=0$. Let $u$ be one of $u_{ \pm}$and $b$ be the corresponding $b_{ \pm}$, respectively. Then we have:

- $|u-b| \leqslant c \epsilon^{1+\alpha}$;

- $f_{B(x, \epsilon)}|\nabla(u-b)|^{2} \leqslant c \epsilon^{\alpha}$;

- $f_{B(x, \epsilon)}\left|\operatorname{Hess}_{u}\right|^{2}<c \epsilon^{-2+\alpha}$.

Here $\alpha=\alpha(n)$ is a universal constant, which can be chosen as $1 /(2 n-1)$.

Proof. For simplicity, we assume $u=u_{+}$and $b=b_{+}$.

The pointwise estimate of $|u-b|$ follows from maximum principle and the excess estimate (2.68), same as traditional case.

We proceed to show the integral estimate of $|\nabla(u-b)|$. Note that $b(x)=$ $r(x)-d\left(x_{0}, p_{+}\right)$, where $r(x)=d\left(x, p_{+}\right)$. It follows from rescaling that

$$
f_{B\left(x_{0}, 4 \epsilon\right)}|\Delta b|=f_{B\left(x_{0}, 4 \epsilon\right)}|\Delta r|<C \epsilon^{-1}
$$

Actually, by the fact $\Delta r \leqslant(2 n-1) / r$, the estimate of $\int|\Delta r|$ is reduced to the estimate of $\int \Delta r$. However, $\int \Delta r$ can be bounded by integration by parts, modulo some technical discussion around the generalized cut locus and singular set $\mathcal{S}$. Due to the high codimension of $\mathcal{S}$, the integral of $\Delta r$ around of $\mathcal{S}$ can be ignored. Then we return to the smooth manifold case, which is discussed clearly in [6]. 
Clearly, $u-b \in N_{0}^{1,2}\left(B\left(x_{0}, 4 \epsilon\right)\right)$. Hence integration by parts, Proposition 2.17, applies and we have

$$
\begin{aligned}
f_{B\left(x_{0}, 4 \epsilon\right)}|\nabla(u-b)|^{2} & =f_{B\left(x_{0}, 4 \epsilon\right)}(u-b) \Delta(b-u)=f_{B\left(x_{0}, 4 \epsilon\right)}(u-b) \Delta b \\
& \leqslant C \epsilon^{1+\alpha} f_{B\left(x_{0}, 4 \epsilon\right)}|\Delta b|<C \epsilon^{\alpha} .
\end{aligned}
$$

Note that $u$ is harmonic in $B\left(x_{0}, 4 \epsilon\right)$. Weitzenböck formula implies that

$$
\frac{1}{2} \Delta\left(|\nabla u|^{2}-1\right)=\frac{1}{2} \Delta|\nabla u|^{2}=\left|\operatorname{Hess}_{u}\right|^{2} \geqslant 0
$$

in the classical sense on $B\left(x_{0}, 4 \epsilon\right) \backslash \mathcal{S}$. By extension property of the subharmonic function, Proposition 2.19, we see that $|\nabla u|^{2} \in N_{\mathrm{loc}}^{2}\left(B\left(x_{0}, 4 \epsilon\right)\right)$. Let $\phi$ be a cutoff function vanishes on $\partial B\left(x_{0}, 4 \epsilon\right)$ and equivalent to 1 on $B\left(x_{0}, \epsilon\right)$, with $\epsilon|\nabla \phi|$ and $\epsilon^{2}|\Delta \phi|$ bounded as in Lemma 2.38. Clearly, $\phi \in N_{c}^{1,2}\left(B\left(x_{0}, 4 \epsilon\right)\right)$. Therefore, it follows from integration by parts, Proposition 2.17, that

$$
2 f_{B\left(x_{0}, 4 \epsilon\right)} \phi\left|\operatorname{Hess}_{u}\right|^{2}=f_{B\left(x_{0}, 4 \epsilon\right)} \phi \Delta\left(|\nabla u|^{2}-1\right)=f_{B\left(x_{0}, 4 \epsilon\right)}\left(|\nabla u|^{2}-1\right) \Delta \phi .
$$

Consequently, we obtain

$$
f_{B\left(x_{0}, \epsilon\right)}\left|\operatorname{Hess}_{u}\right|^{2} \leqslant f_{B\left(x_{0}, 4 \epsilon\right)} \phi\left|\operatorname{Hess}_{u}\right|^{2} \leqslant\left. C \epsilon^{-2} f_{B\left(x_{0}, 4 \epsilon\right)}|| \nabla u\right|^{2}-1 \mid \leqslant C \epsilon^{\alpha-2} . \square
$$

Note that Lemma 2.39 implies almost splitting property already. Therefore, it is a generalization of Lemma 2.31, the splitting property. Not surprisingly, one can use Lemma 2.39 to prove Lemma 2.31, at least formally. Actually, if there is a line with length $2 L$ centered at $x_{0}$, then in the unit ball $B\left(x_{0}, 1\right)$, it follows from Lemma 2.39 that

$$
|u-b|<c L^{-\alpha}, \quad f_{B\left(x_{0}, 1\right)}|\nabla(u-b)|^{2}<c L^{-\alpha}, \quad f_{B\left(x_{0}, 1\right)}\left|\operatorname{Hess}_{u}\right|^{2}<c L^{-\alpha} .
$$

Let $L \rightarrow \infty$, we see that $\operatorname{Hess}_{u} \equiv 0$ on $\mathcal{R}$.

From the proof of Lemma 2.39, it is clear that the key to obtain smallness of $\left|\operatorname{Hess}_{u}\right|^{2}$ is the integration by parts, which is checked in our case. For smooth Riemannian manifold, the approximation in Lemma 2.39 was improved by Colding and Naber in [14]. The essential difference is that they chose parabolic approximation functions, instead of harmonic approximations. Suppose $\gamma$ is a line segment with length 2 , centered around $x_{0}$, with end points $p_{+}$and $p_{-}$. Then one can construct cutoff functions $\psi$ such that it vanishes outside $B\left(x_{0}, 8\right)$ and inside 
$B\left(p_{+}, 0.01\right)$ and $B\left(p_{-}, 0.01\right)$, and equals 1 on $B\left(x_{0}, 4\right) \backslash\left(B\left(p_{+}, 0.02\right) \cup B\left(p_{-}\right.\right.$, $0.02))$. Moreover, we have pointwise bound of $|\Delta \psi|$ and $|\nabla \psi|$. Then for $b_{ \pm}$, we can run heat flow starting from $\psi b_{ \pm}$to obtain solution $h_{t, \pm}$. Then the function $h_{t, \pm}$ is a better approximation function of $b_{ \pm}$on the scale around $\sqrt{t}$. The extra technical tools needed for Colding-Naber's argument beyond the harmonic approximation consists of an a priori bound of heat kernel, and the construction of the cutoff function with the properties as mentioned above. However, in light of Proposition 2.20 and Lemma 2.38, both tools are available in our setting. Note also that $h_{t, \pm}$ are bounded heat solutions, so $\left|\nabla h_{t, \pm}\right|^{2} \in N_{\text {loc }}^{1,2}$ and bounded (see Appendix A). Hence, $\square\left|\nabla h_{t, \pm}\right|^{2} \leqslant 0$ in distribution sense and $\left|\nabla h_{t, \pm}\right|^{2}$ satisfy standard mean-value inequality. This guarantees the local pointwise estimate of $\left|\nabla h_{t, \pm}\right|$. Therefore, we can develop our version of the parabolic approximation estimate, [14, Theorem 2.19], in the current case.

LEMMA 2.40 (Parabolic approximation of local Busemann function). There exist two constants $c=c(n), \bar{\epsilon}=\bar{\epsilon}(n)$ with the following properties.

Suppose $x_{0} \in X, \gamma$ is a line segment whose center point locates in $B\left(x_{0}, 0.2 \epsilon\right)$, with end points $p_{+}$and $p_{-}$, with length 2 . Let $h_{t}$ be the heat approximation of $b$ which is one of $b_{ \pm}$. Suppose the excess value $d\left(x_{0}, p_{+}\right)+d\left(x_{0}, p_{-}\right)-2<\epsilon^{2}$ for some $\epsilon \in(0, \bar{\epsilon})$. Then there exists $\lambda \in[0.5,2]$ such that:

- $\left|h_{\lambda \epsilon^{2}}-b\right| \leqslant c \epsilon^{2}$;

- $\left.f_{B(x, \epsilon)}|| \nabla h_{\lambda \epsilon^{2}}\right|^{2}-1 \mid \leqslant c \epsilon$;

- $\left.\int_{0.1}^{1.9} f_{B(x, \epsilon)}|| \nabla h_{\lambda \epsilon^{2}}\right|^{2}-1 \mid \leqslant c \epsilon^{2}$.

Most importantly, we have

$$
\int_{0.1}^{1.9} f_{B(\gamma(s), \epsilon)}\left|\operatorname{Hess}_{h_{\lambda \epsilon^{2}}}\right|^{2} \leqslant c .
$$

Note that we did not formulate the parabolic approximation in the most precise way. For example, $\gamma$ need not to be a geodesic, an $\epsilon$-geodesic suffices. Interested readers are referred to [14] for the most general version.

According to the discussion from Lemma 2.38 to Lemma 2.40, it is quite clear that the integral estimate of approximation functions can be obtained in the same way as the Riemannian manifold case, provided the following properties.

- Almost superharmonicity of distance functions, Proposition 2.30.

- Bishop-Gromov volume comparison, Proposition 2.3. 
- Strong maximum principle for subharmonic functions, Proposition 2.28.

- Integration by parts, Proposition 2.17.

- Existence of excellent cutoff function, Lemma 2.38.

Since all of these properties are checked in our situation, we can follow the route of Cheeger-Colding to obtain the following properties, almost line by line.

LEMMA 2.41 (Approximation slices). Suppose $x_{0} \in X$, and the pointed-GromovHausdorff distance between $\left(X, x_{0}\right)$ and $\left(Y \times \mathbb{R}^{k},(\hat{y}, 0)\right)$ is less than $\psi\left(L^{-1}\right)$ for some metric space $Y$. Let $\gamma_{1}, \gamma_{2}, \ldots, \gamma_{k}$ be $k$ line segments with length $2 L \gg 2$ such that the center point of $\gamma_{i}$ locates in $B\left(x_{0}, 1\right)$ for each $i$. Furthermore, the Gromov-Hausdorff distance between $\gamma_{1} \cup \gamma_{2} \cdots \cup \gamma_{k}$ and $\tilde{\gamma}_{1} \cup \tilde{\gamma}_{2} \cup \cdots \tilde{\gamma}_{k}$ is bounded by $\psi\left(L^{-1}\right)$, where $\tilde{\gamma}_{i}$ is the line segment on the ith coordinate axis of $\mathbb{R}^{k}$, centered at the origin and with length $2 L, \psi$ is a nonnegative monotonically increasing function satisfying $\psi(0)=0$. Suppose the end points of $\gamma_{i}$ are $p_{i,+}$ and $p_{i,-}$. Let $b_{i, \pm}$ be the corresponding local Busemann functions with respect to $\gamma_{i}$. Let $u_{i}$ be the harmonic function on $B\left(x_{i}, 4\right)$ with the same value as $b_{i, \pm}$ on $\partial B\left(x_{0}, 4\right)$. Then we have

$$
\int_{B\left(x_{0}, 1\right)}\left\{\sum_{1 \leqslant i \leqslant k}\left|\nabla u_{i}-1\right|^{2}+\sum_{1 \leqslant i<j \leqslant k}\left|\left\langle\nabla u_{i}, \nabla u_{j}\right\rangle\right|+\sum_{1 \leqslant i \leqslant k}\left|\operatorname{Hess}_{u_{i}}\right|^{2}\right\} \leqslant \bar{\psi}\left(L^{-1}\right),
$$

where $\bar{\psi}$ is also a nonnegative monotonically increasing function satisfying $\bar{\psi}(0)=0$, depending on $\psi$.

Sketch of the proof. In light of Lemma 2.39, it suffices to prove

$$
\int_{B\left(x_{0}, 1\right)}\left|\left\langle\nabla u_{i}, \nabla u_{j}\right\rangle\right| \leqslant \bar{\psi}\left(L^{-1}\right)
$$

for each $\{i, j\}$ with $i<j$. One can follow the argument of Colding [13] and Cheeger [6], with slight modification to deal with the extra trouble caused by the existence of the singularities. For simplicity of notation, we choose $i=1, j=2$. Since the proof has nothing to do with the Kähler condition, we shall prove it in the Riemannian setting and set $m=2 n$ for the simplicity of notations.

Step 1. For each continuous function $f$ which is smooth on $\mathcal{R} \cap B\left(x_{0}, 2\right)$ and has bounded $|\nabla f|$, we have

$$
\frac{1}{\left|S B\left(x_{0}, 1\right)\right|} \int_{S B\left(x_{0}, 1\right)}\left|\langle\nabla f, v\rangle-\left(f\left(\gamma_{v}(1)\right)-f\left(\gamma_{v}(0)\right)\right)\right|<2^{m} f_{B\left(x_{0}, 2\right)}\left|\operatorname{Hess}_{f}\right| .
$$


In particular, let $u$ be one of $u_{1}$ or $u_{2}$, the harmonic approximation functions of $b_{1}$ and $b_{2}$, then we have

$$
\frac{1}{\left|S B\left(x_{0}, 1\right)\right|} \int_{S B\left(x_{0}, 1\right)}\left|\langle\nabla u, v\rangle-\left(u\left(\gamma_{v}(1)\right)-u\left(\gamma_{v}(0)\right)\right)\right|<C L^{-\alpha / 2}
$$

for some $C=C(m)$.

Notice that we use $S B\left(x_{0}, 1\right)$ to denote the unit sphere bundle over $B\left(x_{0}, 1\right) \cap \mathcal{R}$, for simplicity of notations. We also need to make sense of the integral on both sides of (2.70). The right hand side integral actually happens on $B\left(x_{0}, 2\right) \cap \mathcal{R}=$ $B\left(x_{0}, 2\right) \backslash \mathcal{S}$, which is a full measure subset of $B\left(x_{0}, 2\right)$. Since the subset $B\left(x_{0}\right.$, 2) $\cap \mathcal{S}$, where $\left|\mathrm{Hess}_{f}\right|$ is not defined, is only a measure-zero set, we abuse notation by using $\int_{B\left(x_{0}, 2\right)}\left|\operatorname{Hess}_{f}\right|$ to denote $\int_{B\left(x_{0}, 2\right) \backslash \mathcal{S}} \mid$ Hess $_{f} \mid$. The situation of the left hand side of (2.70) is similar. Note that $\gamma_{v}(1)$ may not be defined since it is possible that $\gamma_{v}(t) \in \mathcal{S}$ for some $t<1$ even if $v \in T_{x}(X)$ for some regular point $x \in B\left(x_{0}\right.$, $1) \backslash \mathcal{S}$. Then the geodesic cannot proceed beyond $t$. We call $v$ to be exceptional if $\gamma_{v}(t) \in \mathcal{S}$ for some $t \in[0,1]$. We collect all such exceptional $v$ 's together and call the collection as the exceptional set, denoted by $E$. In general, $E \neq \emptyset$. However, it is not hard to see that $E$ is a measure-zero subset of $S B\left(x_{0}, 1\right)$.

Actually, due to the high codimension of $\mathcal{S}$, following the argument of the proof of Lemma 2.31, for each small number $\xi$, we can find a smooth hypersurface $\Sigma_{\xi}$ (see Claim 2.32) such that

$$
\left|B\left(x_{0}, 10\right) \cap \Sigma_{\xi}\right| \leqslant C \xi ; \quad \frac{1}{C} \xi<d(x, \mathcal{S})<C \xi, \quad \forall x \in \Sigma_{\xi} \cap B\left(x_{0}, 10\right) .
$$

Here $C$ may depend on $x_{0}$. Let $E_{\xi}$ be the subset of $S\left(B\left(x_{0}, 1\right) \cap \mathcal{R}\right)$ such that $\gamma_{v}(t) \in \Sigma_{\xi}$ for some $t \in[0,2]$. Then $E_{\xi}$ can be regarded as a bundle over the $S\left(\Sigma_{\xi} \cap B\left(x_{0}, 2\right)\right)$, the collection of $v \in T_{y} X$ such that $y \in \Sigma_{\xi} \cap B\left(x_{0}, 2\right)$ and $v \in T_{y} \Sigma_{\xi}$. We equip $\left\{\Sigma_{\xi} \cap B\left(x_{0}, 2\right)\right\} \times[0,2]$ with the obvious product measure and define a map $\varphi$ from $E_{\xi}$ to $\left\{\Sigma_{\xi} \cap B\left(x_{0}, 2\right)\right\} \times[0,2]$ as follows:

$$
\begin{aligned}
\varphi: E_{\xi} & \mapsto S\left\{\Sigma_{\xi} \cap B\left(x_{0}, 10\right)\right\} \times[0,2] \\
v & \mapsto\left(\gamma_{v}^{\prime}\left(t_{v}\right), t_{v}\right),
\end{aligned}
$$

where $t_{v}$ is the first time $t$ such that $\gamma_{v}(t) \in \Sigma_{\xi}$. Clearly, $d\left(\gamma_{v}\left(t_{v}\right), \pi(v)\right)<\left|t_{v}\right| \leqslant 2$, it follows from triangle inequality that $\gamma_{v}\left(t_{v}\right) \in B\left(x_{0}, 3\right)$. Therefore, the above map is well defined. In light of Liouville's theorem, the geodesic flow on sphere bundle preserves the volume form, as $\left|\gamma_{v}^{\prime}(t)\right| \equiv 1$, it is clear that $\varphi$ is volume expanding. It follows that

$$
\left|E_{\xi}\right|_{\mathcal{H}^{2 m-1}} \leqslant 2\left|S\left\{\Sigma_{\xi} \cap B\left(x_{0}, 10\right)\right\}\right|_{\mathcal{H}^{2 m-2}} \leqslant C\left|\Sigma_{\xi}\right|_{\mathcal{H}^{m-1}} \leqslant C \xi .
$$


Suppose $v \in E$, then $v \in T_{x} X$ for some $x \in B\left(x_{0}, 1\right) \cap \mathcal{R}$ and $\gamma_{v}\left(t_{0}\right) \in \mathcal{S}$ for some $t_{0} \in[0,1]$. Recall that $\Sigma_{x 1}$ can be regarded as the boundary of $\xi$-neighborhood of $\mathcal{S}$, then it follows from connectedness of $\gamma$ that $\gamma(s) \in \Sigma_{\xi}$ for some $s \in[0$, $\left.t_{0}\right] \subset[0,2]$. Consequently, $v \in E_{\xi}$. This means that $E \subset E_{\xi}$ for every small positive $\xi$. It follows from (2.72) that $E$ is a measure-zero subset, of the sphere bundle over $B\left(x_{0}, 1\right) \cap \mathcal{R}$, which is denoted by $S B\left(x_{0}, 1\right)$ for simplicity.

We proceed to prove (2.70). For each $v \in S B\left(x_{0}, 1\right) \backslash E$, the intermediate value theorem implies

$$
f\left(\gamma_{v}(1)\right)-f\left(\gamma_{v}(0)\right)=\left(f \circ \gamma_{v}\right)^{\prime}\left(t_{0}\right)
$$

for some $t_{0} \in[0,1]$. Consequently, we have

$$
\begin{aligned}
& \langle\nabla f, v\rangle-\left(f\left(\gamma_{v}(1)\right)-f\left(\gamma_{v}(0)\right)\right)=-\left(f \circ \gamma_{v}\right)^{\prime}\left(t_{0}\right)+\left(f \circ \gamma_{v}\right)^{\prime}(0) \\
& \quad=-\int_{0}^{t_{0}}(f \circ \gamma)^{\prime} d t=-\int_{0}^{t_{0}} \int_{0}^{t} \frac{\partial^{2}}{\partial \tau^{2}}(f \circ \gamma) d \tau d t .
\end{aligned}
$$

Taking absolute value on both sides yields that

$$
\left|\langle\nabla f, v\rangle-\left(f\left(\gamma_{v}(1)\right)-f\left(\gamma_{v}(0)\right)\right)\right| \leqslant t_{0} \int_{0}^{t_{0}}\left|\operatorname{Hess}_{f}\right| d t \leqslant \int_{0}^{1}\left|\operatorname{Hess}_{f}\right| d t .
$$

Integrating both sides of the above inequality on $S B\left(x_{0}, 1\right) \backslash E$, we obtain

$$
\begin{aligned}
& \int_{S B\left(x_{0}, 1\right) \backslash E}\left|\langle\nabla f, v\rangle-\left(f\left(\gamma_{v}(1)\right)-f\left(\gamma_{v}(0)\right)\right)\right| \\
& \quad \leqslant \int_{S B\left(x_{0}, 1\right) \backslash E}\left(\int_{0}^{1}\left|\operatorname{Hess}_{f}\right| d t\right) \leqslant \int_{0}^{1}\left\{\int_{S B\left(x_{0}, 2\right) \backslash E}\left|\operatorname{Hess}_{f}\right|\right\} d t \\
& \quad=\int_{S B\left(x_{0}, 2\right) \backslash E}\left|\operatorname{Hess}_{f}\right| .
\end{aligned}
$$

Note that we have used the fact that the geodesic flow is volume preserving in the above inequality. It is clear that

$$
\int_{S B\left(x_{0}, 2\right) \backslash E}\left|\operatorname{Hess}_{f}\right|=\left|S_{m-1}\right| \int_{B\left(x_{0}, 2\right) \backslash \mathcal{S}}\left|\operatorname{Hess}_{f}\right|=m \omega_{m} \int_{B\left(x_{0}, 2\right) \backslash \mathcal{S}}\left|\operatorname{Hess}_{f}\right| .
$$

By abuse of notation, the combination of the previous inequalities implies that

$$
\begin{aligned}
& \frac{1}{\left|S B\left(x_{0}, 1\right)\right|} \int_{S B\left(x_{0}, 1\right)}\left|\langle\nabla f, v\rangle-\left(f\left(\gamma_{v}(1)\right)-f\left(\gamma_{v}(0)\right)\right)\right| \\
& <\frac{m \omega_{m}}{\left|S B\left(x_{0}, 1\right)\right|} \int_{B\left(x_{0}, 2\right)}\left|\operatorname{Hess}_{f}\right|=\frac{1}{\left|B\left(x_{0}, 1\right)\right|} \int_{B\left(x_{0}, 2\right)}\left|\operatorname{Hess}_{f}\right| \\
& =\frac{\left|B\left(x_{0}, 2\right)\right|}{\left|B\left(x_{0}, 1\right)\right|} f_{B\left(x_{0}, 2\right)}\left|\operatorname{Hess}_{f}\right| \leqslant 2^{m} f_{B\left(x_{0}, 2\right)}\left|\operatorname{Hess}_{f}\right|,
\end{aligned}
$$


where the last step follows from the Bishop-Gromov volume comparison. The above inequality is nothing but (2.70). Clearly, (2.71) follows from Lemma 2.39 and (2.70). Therefore, we finish the proof of Step 1.

We remind the reader that the proof of step 1 is almost the same as [13, Proposition 1.32]. The proofs of the following steps are also standard and exactly the same as the one in [13]. So we just describe the basic statements and omit the details.

Step 2. Let $\mathbf{U}=\left(u_{1}, u_{2}\right)$. For every pair of points $x, y \in B\left(x_{0}, 3\right)$, we have

$$
|\mathbf{U}(x)-\mathbf{U}(y)|<d(x, y)+\xi
$$

for some $\xi=\xi\left(L^{-1}, \psi \mid m\right)$.

Let us explain the rough motivation behind (2.73). Actually, it follows from the Gromov-Hausdorff closeness between $\left(X, x_{0}\right)$ and $\left(Y \times \mathbb{R}^{k},(\hat{y}, 0)\right)$ that $\left(b_{1}, b_{2}\right)$ is an almost submersion from $B\left(x_{0}, 10\right)$ to its image in $\mathbb{R}^{2}$. Then we apply the $C^{0}$-closeness between $b_{i}$ and $u_{i}$, we see that the map $\left(u_{1}, u_{2}\right)$ is also an almost submersion. An almost submersion almost decreases distance, which is the meaning of (2.73). This can be proved by elementary methods, following the definition of Gromov-Hausdorff distance.

Step 3. Fix $\theta>0$ small and set

$$
C_{\theta} \triangleq\left\{v \in S B\left(x_{0}, 1\right) \mid \angle\left(v, \nabla u_{1}\right)<\theta\right\} .
$$

Then we have

$$
\int_{C_{\theta}}\left|u_{2}\left(\gamma_{v}(1)\right)-u_{2}\left(\gamma_{v}(0)\right)\right| \leqslant 2 \theta\left|C_{\theta}\right|
$$

whenever $L>L_{0}(m, \theta)$.

Step 4. We have

$$
\int_{C_{\theta}}\left|\left\langle\nabla u_{2}, v\right\rangle\right| \leqslant 3 \theta\left|C_{\theta}\right|
$$

whenever $L>L_{1}(m, \theta)$.

Step 5. We have

$$
f_{B\left(x_{0}, 1\right)}\left|\left\langle\nabla u_{1}, \nabla u_{2}\right\rangle\right|<5 \theta
$$

whenever $L>L_{1}(m, \theta)$.

The inequality (2.69) follows from (2.77) since $\theta$ can be arbitrarily small, whenever $L$ is very large. 
Let $\vec{u}=\left(u_{1}, u_{2}, \ldots, u_{k}\right)$, we can regard $\vec{u}$ as an almost submersion from $B\left(x_{0}, 1\right)$ to its image on $\mathbb{R}^{k}$. Consequently, slice argument (see [10, Section 2]) can be set up. A fundamental application of the slice argument is to set up the following volume convergence property, as done in [13] and [6].

Proposition 2.42 (Volume continuity). For every $\left(X, x_{0}, g\right) \in \widetilde{\mathscr{K} \mathscr{S}}(n, \kappa)$ and $\epsilon>0$, there is a constant $\xi=\xi(X, \epsilon)$ such that

$$
\left|\log \frac{\left|B\left(y_{0}, 1\right)\right|}{\left|B\left(x_{0}, 1\right)\right|}\right|<\epsilon
$$

for any $\left(Y, y_{0}, h\right) \in \widetilde{\mathscr{K} \mathscr{S}}(n, \kappa)$ satisfying $d_{\mathrm{PGH}}\left(\left(X, x_{0}, g\right),\left(Y, y_{0}, h\right)\right)<\xi$.

Recall that $d_{\mathrm{PGH}}$ means the pointed-Gromov-Hausdorff distance. In Proposition 2.42, the inequality $d_{\mathrm{PGH}}\left(\left(X, x_{0}, g\right),\left(Y, y_{0}, h\right)\right)<\xi$ means that the Gromov-Hausdorff distance between $B\left(x_{0}, \xi^{-1}\right) \subset X$ and $B\left(y_{0}, \xi^{-1}\right) \subset Y$ is less than $\xi$.

Sketch of the proof. It suffices to prove the following statement.

Suppose $\left(Y_{i}, y_{i}, h_{i}\right) \in \widetilde{\mathscr{K} \mathscr{S}}(n, \kappa)$ converges to $\left(X, x_{0}, g\right) \in \widetilde{\mathscr{K} \mathscr{S}}(n, \kappa)$ in the pointed-Gromov-Hausdorff topology, then we have

$$
\lim _{i \rightarrow \infty}\left|B\left(y_{i}, 1\right)\right|=\left|B\left(x_{0}, 1\right)\right| .
$$

Let $\mathcal{S}_{r}$ be the $r$-neighborhood of $\mathcal{S} \cap B\left(x_{0}, 1\right)$. Then it follows from the definition of Minkowski dimension and the fact $\operatorname{dim}_{\mathcal{M}} \mathcal{S}<2 n-3$ that

$$
\left|\mathcal{S}_{r}\right| \leqslant C r^{3}
$$

for some $C$ depending on $\left(X, x_{0}, g\right)$. Up to a covering argument, the volume convergence is then reduced to the volume convergence on the uniform regular part $B\left(x_{0}, 1\right) \backslash \mathcal{S}_{r}$ for each small positive $r$. Then we can follow the argument of Colding [13, Theorem 0.1$]$ to finish the proof, whenever the following statement is available:

Given $\epsilon>0$, there exists $L=L(\epsilon, n)>1$ such that

$$
|| B\left(x_{0}, 1\right)\left|-\omega_{2 n}\right|<\epsilon
$$

whenever $d_{G H}\left(B\left(x_{0}, L\right), B(0, L)\right)<L^{-1}$. Here $B(0, L)$ is the standard ball of radius $L$ in $\mathbb{R}^{2 n}, B\left(x_{0}, L\right)$ is a geodesic ball of radius $L$ in some $Y \in \widetilde{\mathscr{K} \mathscr{S}}(n, \kappa)$.

The smooth version of the above statement is Colding [13, Lemma 2.1], whose proof used directly the multi-Busemann functions. The proof was refined by 
Cheeger (see [6, Theorem 9.31 on page 44]), replacing the Busemann functions by their harmonic approximations and using the slicing estimate (see Lemma 2.41) for $k=2 n$. One key new ingredient of Cheeger's proof is to make use of the mod-2 degree theory. However, due to the high codimension of $\mathcal{S}$, it is clear that $X \backslash \mathcal{S}$ is a connected manifold. Then it is not hard to see the mod-2 degree theory works in the current setting for the bounded harmonic map $\vec{u}$. So we can follow the route of Cheeger (see [6, Theorem 9.31]) to prove the above statement.

Applying the same argument as in [8, Theorem 4.85] or [6, Theorem 9.45], we obtain the almost rigidity of volume cones (see Lemma 2.34 for the precise rigidity of volume cone).

Proposition 2.43 (Almost volume cone implies almost metric cone). For each $\epsilon>0$, there exists $\xi=\xi(\epsilon \mid n)$ with the following properties.

Suppose $\left(X, x_{0}, g\right) \in \widetilde{\mathscr{K} \mathscr{S}}(n, \kappa)$ satisfies $\left|B\left(x_{0}, 2\right)\right| /\left|B\left(x_{0}, 1\right)\right| \geqslant(1-\epsilon) 2^{2 n}$, then there exists a metric cone over a length space $Z$, with vertex $z^{*}$ such that

$$
\operatorname{diam}(Z)<\pi+\xi, \quad d_{G H}\left(B\left(x_{0}, 1\right), B\left(z^{*}, 1\right)\right)<\xi .
$$

The notation $\xi(\epsilon \mid n)$ means that $\lim _{\epsilon \rightarrow 0} \xi(\epsilon \mid n)=0$. Notice that Proposition 2.43 is an almost version of Lemma 2.34. Similar to the fact that Lemma 2.39 is developed to achieve the almost splitting, the following effective estimates can be developed to realize Proposition 2.43.

For each small positive number $\epsilon$, there exist small numbers $\xi_{1}(\epsilon \mid n)$ and $\xi_{2}(\epsilon \mid n)$ with the following properties.

Suppose $X \in \widehat{\mathscr{K} \mathscr{S}}(n, \kappa), x_{0} \in X$. Suppose $\left|B\left(x_{0}, 2\right)\right| /\left|B\left(x_{0}, 1\right)\right| \geqslant(1-\epsilon) 2^{2 n}$. Then there exists an $r_{0} \in(1.5,2)$ such that

$$
\frac{r_{0}\left|\partial B\left(x_{0}, r_{0}\right)\right|}{\left|B\left(x_{0}, r_{0}\right)\right|} \geqslant 2 n-8 \epsilon .
$$

Fix $r_{0}$ and let $w$ be the solution of the Dirichlet Poisson equation

$$
\begin{cases}\Delta w=2 n & \text { in } B\left(x_{0}, r_{0}\right) \\ w=\frac{r_{0}^{2}}{2} & \text { on } \partial B\left(x_{0}, r_{0}\right)\end{cases}
$$

Let $r$ be the distance function to $x_{0}$. Then we have

$$
\left|2 w-r^{2}\right|_{L^{\infty}\left(B\left(x_{0}, 1\right)\right)}+f_{B\left(x_{0}, 1\right) \backslash \mathcal{S}}|| \nabla w|-r|+f_{B\left(x_{0}, 1\right) \backslash \mathcal{S}}\left|\operatorname{Hess}_{w}-g\right|^{2}<\xi_{1} .
$$


Furthermore, on $B\left(x_{0}, 1\right) \backslash \mathcal{S}$, we have

$$
|\nabla w|-r<\xi_{2}
$$

The proof of the above estimates is similar to that of Lemma 2.39. Under the help of maximum principle (see Proposition 2.28), the existence of well-behaved radial function (see Lemma 2.36) and segment inequality (see Proposition 2.6), it can be finished exactly as the proof of Cheeger-Colding (see [8, Theorem 4.85]).

Now we use the Kähler condition. Similar to [10, Lemma 9.14], we can derive the almost Kähler cone splitting (see Lemma 2.35 for the precise Kähler cone splitting) from Proposition 2.43.

Proposition 2.44 (Almost Kähler cone splitting). For each $\epsilon>0$, there exists $\xi=\xi(\epsilon \mid n)$ with the following properties.

Suppose $X \in \widetilde{\mathscr{K} \mathscr{S}}(n, \kappa), x_{0} \in X, b$ is a smooth function on $B\left(x_{0}, 2\right)$ satisfying

$$
\sup _{B\left(x_{0}, 2\right) \backslash \mathcal{S}}|\nabla b| \leqslant 2, \quad f_{B\left(x_{0}, 2\right) \backslash \mathcal{S}}\left|\operatorname{Hess}_{b}\right|^{2} \leqslant \epsilon^{2} .
$$

Suppose also $\left|B\left(x_{0}, 2\right)\right| /\left|B\left(x_{0}, 1\right)\right| \geqslant(1-\epsilon) 2^{2 n}$, that is, $B\left(x_{0}, 1\right)$ is an almost volume cone. Then there exists a smooth function $\tilde{b}$ on $B\left(x_{0}, 1\right) \backslash \mathcal{S}$ such that

$$
\sup _{B\left(x_{0}, 1\right) \backslash \mathcal{S}}|\tilde{b}| \leqslant 3, \quad f_{B\left(x_{0}, 1\right) \backslash \mathcal{S}}|\nabla \tilde{b}-J \nabla b|^{2} \leqslant \xi .
$$

Sketch of the proof. Let $w$ be the function constructed in (2.82). On $B\left(x_{0}, 1\right) \backslash \mathcal{S}$, we define

$$
\tilde{b} \triangleq\langle J \nabla b, \nabla w\rangle
$$

where $J$ is the complex structure on $\mathcal{R}$. Notice that

$$
\Delta\left\{|\nabla w|^{2}-2 w\right\}=2\left|\operatorname{Hess}_{w}-g\right|^{2}
$$

on regular part. In light of (2.81), (2.82) and integration by parts, it is easy to check that $\tilde{b}$ satisfies the desired property.

2.5. Volume radius. Anderson's gap theorem implies that one can improve regularity of the interior part of a geodesic ball whenever the volume ratio of the geodesic ball is very close to the Euclidean one. This suggests us to define the volume radius as follows. 
Definition 2.45. Let $\delta_{0}$ be the Anderson constant. Suppose $X \in \widetilde{\mathscr{K} \mathscr{S}}(n, \kappa)$, $x_{0} \in X$. Then we define

$$
\begin{aligned}
\Omega_{x_{0}} & \triangleq\left\{r\left|r>0, r^{-2 n}\right| B\left(x_{0}, r\right) \mid \geqslant\left(1-\delta_{0}\right) \omega_{2 n}\right\}, \\
\operatorname{vr}\left(x_{0}\right) & \triangleq \begin{cases}\sup \Omega_{x_{0}} & \text { if } \Omega_{x_{0}} \neq \emptyset, \\
0 & \text { if } \Omega_{x_{0}}=\emptyset .\end{cases}
\end{aligned}
$$

We call $\operatorname{vr}\left(x_{0}\right)$ the volume radius of the point $x_{0}$.

According to this definition, a point is regular if and only if its volume radius is positive. On the other hand, if the space is not $\mathbb{C}^{n}$, then every point has a finite volume radius by a generalized Anderson's gap theorem.

Proposition 2.46 (Euclidean space by vr). Suppose $X \in \widetilde{\mathscr{K} \mathscr{S}}(n)$ and $\operatorname{vr}\left(x_{0}\right)=\infty$ for some $x_{0} \in X$, then $X$ is isometric to the Euclidean space $\mathbb{C}^{n}$.

Proof. Fix an arbitrary point $x \in X$, then volume comparison implies that

$$
\mathrm{v}(x) \geqslant \lim _{r \rightarrow \infty} \omega_{2 n}^{-1} r^{-2 n}|B(x, r)|=\operatorname{avr}(X) \geqslant 1-\delta_{0} .
$$

Therefore, $x$ is a regular point. Since $x$ is arbitrarily chosen, we see that $X \in$ $\mathscr{K} \mathscr{S}(n)$. Then the statement follows from Anderson's gap theorem.

A local version of Proposition 2.46 is the following local Harnack inequality of vr.

PROPOSITION 2.47 (Local Harnack inequality of volume radius). There is a constant $\tilde{K}=\tilde{K}(n)$ with the following properties.

Suppose $x \in X \in \widetilde{\mathscr{K} \mathscr{S}}^{*}(n), r=\operatorname{vr}(x)>0$, then we have

$$
\tilde{K}^{-1} r \leqslant \mathbf{v r} \leqslant \tilde{K} r
$$

in the ball $B\left(x, \tilde{K}^{-1} r\right)$. Moreover, for every $\rho \in\left(0, \tilde{K}^{-1} r\right), y \in B\left(x, \tilde{K}^{-1} r\right)$, we have

$$
\begin{aligned}
\omega_{2 n}^{-1} \rho^{-2 n}|B(y, \rho)| & \geqslant 1-\frac{\delta_{0}}{100}, \\
|R m|(y) & \leqslant \tilde{K}^{2} r^{-2} \\
\operatorname{inj}(y) & \geqslant \tilde{K}^{-1} r .
\end{aligned}
$$


Proof. It follows from Bishop volume comparison, Anderson's gap theorem and a compactness argument. Actually, by adjusting $\tilde{K}$ if necessary, it suffices to show (2.85). We argue by contradiction. Suppose (2.85) were wrong, by point-selecting and rescaling, we can find a sequence of $L_{i} \rightarrow \infty$ and Ricci-flat spaces $\left(X_{i}, x_{i}\right.$, $\left.g_{i}\right) \in \widetilde{\mathscr{K} \mathscr{S}}^{*}(n)$ such that

$$
|R m|\left(x_{i}\right)=1, \quad \sup _{x \in B\left(x_{i}, L_{i}\right)}|R m|(x) \leqslant 2, \quad \omega_{2 n}^{-1} L_{i}^{-2 n}\left|B\left(x_{i}, L_{i}\right)\right| \geqslant 1-\delta_{0} .
$$

Improving regularity property of Ricci-flat metrics implies higher order estimate of $R m$ in the balls $B\left(x_{i}, L_{i}-1\right)$. Therefore, we can take smooth convergence limit (see [29]):

$$
\left(X_{i}, x_{i}, g_{i}\right) \stackrel{C^{\infty} \text {-Cheeger-Gromov }}{\longrightarrow}\left(X_{\infty}, x_{\infty}, g_{\infty}\right) .
$$

The limit space satisfying $|\operatorname{Rm}|\left(x_{\infty}\right)=1$ and $\operatorname{avr}\left(X_{\infty}\right) \geqslant 1-\delta_{0}$, which is impossible by Anderson's gap theorem or Proposition 2.46.

On a Ricci-flat geodesic ball, it is well known that $|R m|$ bound implies bound of $\left|\nabla^{k} R m\right|$ for each positive integer $k$ in a smaller geodesic ball. So (2.85) immediately yields the following corollary.

COROLLARY 2.48 (Improving regularity property of volume radius). There is a small positive constant $c_{a}=c_{a}(n)$ with the following properties.

Suppose $x \in X \in{\widetilde{\mathscr{K} \mathscr{S}^{*}}}^{*}(n), \operatorname{vr}(x) \geqslant r>0$, then we have

$$
r^{2+k}\left|\nabla^{k} R m\right|(y) \leqslant c_{a}^{-2}, \quad \forall y \in B\left(x, c_{a} r\right), \quad 0 \leqslant k \leqslant 5 .
$$

In the study of Riemannian manifolds with bounded Ricci curvature, harmonic radius (see [2]) plays an important role. A point $x$ is defined to have harmonic radius at least $r$ if on the smooth geodesic ball $B(x, r)$, there exists a harmonic diffeomorphism $\Psi=\left(u_{1}, u_{2}, \ldots, u_{2 n}\right): B(x, r) \rightarrow \Omega \subset \mathbb{R}^{2 n}$ such that

$$
\frac{1}{2} \delta_{i j} \leqslant g_{i j}=g\left(\nabla u_{i}, \nabla u_{j}\right) \leqslant 2 \delta_{i j}, \quad r^{\frac{3}{2}}\left\|g_{i j}\right\|_{C^{1, \frac{1}{2}}} \leqslant 2 .
$$

Then harmonic radius is defined as the supreme of all the possible $r$ 's mentioned above. For convenience, we use hr to denote harmonic radius. This definition can be easily moved to our case when the underlying space is in $\widetilde{\mathscr{K} \mathscr{S}}^{*}(n)$. We define $\mathbf{h r}$ to be 0 on the singular part of the underlying space. It is clear from the definitions and Proposition 2.47 that volume radius and harmonic radius can bound each other, that is, they are equivalent. The following Proposition is obvious. 
Proposition 2.49 (Equivalence of volume and harmonic radius). Suppose $x \in$ $X \in \widetilde{\mathscr{K} \mathscr{S}}^{*}(n)$, then we have

$$
\frac{1}{C} \mathbf{h r}(x) \leqslant \operatorname{vr}(x) \leqslant C \mathbf{h r}(x)
$$

for some uniform constant $C=C(n)$.

Note that the regularity requirement of the underlying space to define volume radius is much weaker than that to define harmonic radius a priori. Therefore, Proposition 2.49 already implies a regularity improvement. We shall set up the compactness theory based on volume radius, since volume radius may be applicable to more general metric measure spaces.

Let $X \in \widetilde{\mathscr{K} \mathscr{S}}^{*}(n)$ and decompose it as $X=\mathcal{R} \cup \mathcal{S}$. Then vr is a positive finite function on $\mathcal{R}$ and equals 0 on $\mathcal{S}$.

Proposition 2.50 (Rigidity of volume ratio). Suppose $X \in \widetilde{\mathscr{K} \mathscr{S}}(n)$. If for two concentric geodesic balls $B\left(x_{0}, r_{1}\right) \subset B\left(x_{0}, r_{2}\right)$ centered at a regular point $x_{0}$, we have

$$
\omega_{2 n}^{-1} r_{1}^{-2 n}\left|B\left(x_{0}, r_{1}\right)\right|=\omega_{2 n}^{-1} r_{2}^{-2 n}\left|B\left(x_{0}, r_{2}\right)\right|,
$$

then the ball $B\left(x_{0}, r_{2}\right)$ is isometric to a geodesic ball of radius $r_{2}$ in $\mathbb{C}^{n}$. Furthermore, if $X \in \mathscr{K} \mathscr{S}(n)$, then we can further conclude that $X$ is Euclidean.

Proof. From the proof of Lemma 2.34, it is clear that $B\left(x_{0}, r_{2}\right)$ is a volume cone with constant volume ratio $\omega_{2 n}$. Observe the change of volume element along each smooth geodesic emanating from $x_{0}$, in the polar coordinate. By the volume density gap between regular and singular points, the optimal volume ratio of $B\left(x_{0}, 1\right)$ forces that it does not contain any singular point. Then the situation is the same as the smooth Riemannian case. Clearly, a smooth Ricci-flat geodesic ball with volume ratio $\omega_{2 n}$ is isometric to a Euclidean ball of the same radius.

If $X \in \mathscr{K} \mathscr{S}(n)$, by analyticity of metric tensor, it is clear that $X$ is flat and hence $\mathbb{C}^{n}$ due to its noncollapsing property at infinity.

PROPOSITION 2.51 (Continuity of volume radius). vr is a continuous function on $X$ whenever $X \in \widetilde{\mathscr{K} \mathscr{S}}(n)$.

Proof. Since vr $\equiv \infty$ on $\mathbb{C}^{n}$, which is obvious continuous. So we can assume $X \in \widetilde{\mathscr{K} \mathscr{S}}^{*}(n)$ without loss of generality. By Proposition 2.46, we know vr is a finite function on $X$. 
So we assume vr is a function with value in $[0, \infty)$. It is also easy to see that vr is continuous at singular points. We know that a point $x_{0}$ is singular if and only if $\operatorname{vr}\left(x_{0}\right)=0$. Clearly, for every sequence $x_{i} \rightarrow x_{0}$, we must have $\lim _{i \rightarrow \infty} \operatorname{vr}\left(x_{i}\right)=0$. Otherwise, we have a sequence $x_{i}$ converging to $x_{0}$ and $\lim _{i \rightarrow \infty} \operatorname{vr}\left(x_{i}\right) \geqslant \xi>0$. However, we note that $x_{0} \in B\left(x_{i}, \tilde{K}^{-1} \xi\right)$ for large $i$. Therefore, $x_{0}$ is forced to be regular by the improving regularity property of volume radius. Contradiction.

Therefore, discontinuity point must admit positive vr if it does exist. Suppose $x_{0}$ is a discontinuous point of vr. Then we can find a sequence of points $x_{i} \in X$ such that

$$
\begin{aligned}
x_{0} & =\lim _{i \rightarrow \infty} x_{i}, \\
0<\operatorname{vr}\left(x_{0}\right) & =r_{0}<\infty, \\
\lim _{i \rightarrow \infty} \operatorname{vr}\left(x_{i}\right) & \neq r_{0} .
\end{aligned}
$$

Clearly, $\log \mathbf{v r}\left(x_{i}\right)$ are uniformly bounded by Proposition 2.47. So we can assume $\operatorname{vr}\left(x_{i}\right)$ converge to a positive number $\bar{r}$. By volume continuity, we clearly have

$$
\begin{aligned}
\omega_{2 n}^{-1} r_{0}^{-2 n}\left|B\left(x_{0}, r_{0}\right)\right| & =1-\delta_{0}=\lim _{i \rightarrow \infty} \omega_{2 n}^{-1} \mathbf{v r}\left(x_{i}\right)^{-2 n}\left|B\left(x_{i}, \operatorname{vr}\left(x_{i}\right)\right)\right| \\
& =\omega_{2 n}^{-1} \bar{r}^{-2 n}\left|B\left(x_{0}, \bar{r}\right)\right| .
\end{aligned}
$$

Since $\bar{r} \neq r_{0}$, we obtain from Proposition 2.50 that $B\left(x_{0}, r_{0}\right)$ is a ball in a metric cone centered at the vertex. Note that $x_{0}$ is a regular point since $\operatorname{vr}\left(x_{0}\right)>0$. Therefore, $B\left(x_{0}, r_{0}\right)$ is the standard ball in $\mathbb{C}^{n}$ with radius $r_{0}$. Consequently, the normalized volume ratio of $B\left(x_{0}, r_{0}\right)$ is 1 , which contradicts the fact that $\operatorname{vr}\left(x_{0}\right)=r_{0}$ and the definition of volume radius.

The volume radius has better property. It satisfies Harnack inequality in the interior of a length-minimizing geodesic. The Hölder continuity estimate of Colding-Naber (see [14]) can be interpreted by volume radius as follows.

PROPOSITION 2.52 (Global Harnack inequality of volume radius). For every small constant $c$, there is a constant $\epsilon=\epsilon(n, \kappa, c)$ with the following properties.

Suppose $(X, g) \in \widetilde{\mathscr{K} \mathscr{S}}(n, \kappa), x, y \in X, \gamma$ is shortest, unit-speed geodesic connecting $x$ and $y$, with smooth interior parts. Suppose $\gamma(0)=x, \gamma(L)=y$, $L \leqslant r$. If $\mathbf{v r}(y)>c r$, then we have

$$
\operatorname{vr}(\gamma(t))>\epsilon r, \quad \forall t \in[c L, L] .
$$

In particular, if $\min \{\mathbf{v r}(x), \operatorname{vr}(y)\}>c r$, then we have

$$
\operatorname{vr}(\gamma(t))>\epsilon r, \quad \forall t \in[0, L] .
$$


Proof. Clearly, (2.90) follows from (2.89). Therefore, it suffices to prove (2.89) only.

Up to a normalization, we can assume $r=L=1$. So $\gamma$ is the shortest geodesic connecting $x, y$ such that $\gamma(0)=x, \gamma(1)=y$. By assumption, we have $\operatorname{vr}(y)>c$. By local Harnack inequality of volume radius, Proposition 2.47, there exists $\bar{\epsilon}=$ $\bar{\epsilon}(n, c)$ such that $\mathbf{v r}>\bar{\epsilon}$ for each $\gamma(t)$ with $t \in[1-\bar{\epsilon}, 1]$. Clearly, for every $t \in[\bar{\epsilon}$, $1-\bar{\epsilon}]$, we have $|\Delta r|<C / \bar{\epsilon}$ for a universal $C=C(n)$, where $r$ is the distance to $\gamma(0)$. Because of the segment inequality (Proposition 2.6) and the parabolic approximation (Lemma 2.40), we can follow the proof of Proposition 3.6 and Theorem 1.1 of [14] verbatim. Similar to the statement in the proof of Theorem 1.1 on page 1213 of [14], we can find constants $\bar{s}=\bar{s}(n, c, \bar{\epsilon}), \bar{r}=\bar{r}(n, c, \bar{\epsilon})$ such that for every $t_{1}, t_{2} \in[\bar{\epsilon}, 1-\bar{\epsilon}]$ satisfying $\left|t_{1}-t_{2}\right|<\bar{s}$ and every $r \in(0, \bar{r})$, we have

$$
1-\frac{\delta_{0}}{100} \leqslant \frac{\left|B\left(\gamma\left(t_{1}\right), r\right)\right|}{\left|B\left(\gamma\left(t_{2}\right), r\right)\right|} \leqslant 1+\frac{\delta_{0}}{100} .
$$

Then it is easy to see that if the volume radius is uniformly bounded below at $t_{1}$, it must be uniformly bounded below at $t_{2}$. Actually, suppose the volume radius at $\gamma\left(t_{1}\right)$ is greater than $r_{1}$ for some $r_{1} \in(0, \bar{r})$, by inequality (2.84) in Proposition 2.47, we have $\omega_{2 n}^{-1} r^{-2 n}\left|B\left(\gamma\left(t_{1}\right), r\right)\right| \geqslant 1-\left(\delta_{0} / 100\right)$ for every $r \in[0$, $\left.r_{1} / \tilde{K}\right]$. Put this information into the above inequality implies that

$$
\omega_{2 n}^{-1} r^{-2 n}\left|B\left(\gamma\left(t_{2}\right), r\right)\right| \geqslant \frac{1-\frac{\delta_{0}}{100}}{1+\frac{\delta_{0}}{100}}>1-\delta_{0}, \quad \forall r \in\left[0, \frac{r_{1}}{\tilde{K}}\right] .
$$

Therefore, the volume radius of $\gamma\left(t_{2}\right)$ is at least $r_{1} / \tilde{K}$. From this induction, it is clear that

$$
\operatorname{vr}(\gamma(t)) \geqslant \tilde{K}^{-(1-\bar{\epsilon}-t) / \bar{s}} \mathbf{v r}(\gamma(1-\bar{\epsilon}))>\bar{\epsilon} \tilde{K}^{-(1-\bar{\epsilon}-t) / \bar{s}} .
$$

Let $\epsilon$ be the number on the right hand side of the above inequality when $t=\bar{\epsilon}$. Then $\epsilon=\epsilon(\bar{\epsilon}, \tilde{K}, \bar{s})=\epsilon(n, \kappa, c)$ and we finish the proof of (2.89).

In general, if $X$ is only a metric space, we even do not know whether vr is semicontinuous. The continuity of vr on $X$ whenever $X \in \widetilde{\mathscr{K} \mathscr{S}}(n, \kappa)$ makes vr a convenient tool to study the geometry of $X$. By Proposition 2.47, one can improve regularity on a scale proportional to vr. So it is convenient to decompose the space $X$ using the function vr.

Definition 2.53. Suppose $(X, g) \in \widetilde{\mathscr{K} \mathscr{S}}(n, \kappa)$. Define

$$
\mathcal{F}_{r}(X) \triangleq\{x \in X \mid \operatorname{vr}(x) \geqslant r\}
$$




$$
\mathcal{D}_{r}(X) \triangleq\left(\mathcal{F}_{r}(X)\right)^{c}=\{x \in X \mid \mathbf{v r}(x)<r\} .
$$

We call $\mathcal{F}_{r}(X)$ the $r$-regular part of $X, \mathcal{D}_{r}(X)$ the $r$-singular part of $X$.

From Definition 2.53, it is clear that

$$
\begin{aligned}
& \mathcal{R}(X)=\bigcup_{r>0} \mathcal{F}_{r}(X), \\
& \mathcal{S}(X)=\bigcap_{r>0} \mathcal{D}_{r}(X) .
\end{aligned}
$$

We observe that the volume radius of each point is related to its distance to singular set by the following property.

Proposition 2.54 (vr bounded from above by distance to $\mathcal{S}$ ). Suppose that

$$
(X, x, g) \in \widetilde{\mathscr{K} \mathscr{S}}(n, \kappa),
$$

$r$ is a positive number. Then

$$
\begin{aligned}
& \{x \mid d(x, \mathcal{S}) \geqslant r\} \supset \mathcal{F}_{\tilde{K} r}, \\
& \{x \mid d(x, \mathcal{S})<r\} \subset \mathcal{D}_{\tilde{K} r} .
\end{aligned}
$$

Proof. Choose an arbitrary point $x \in \mathcal{F}_{\tilde{K} r}$, then $\mathbf{v r}(x) \geqslant \tilde{K} r$. It follows from Proposition 2.57 that $\operatorname{vr}(y) \geqslant r>0$ for every point $y \in B(x, r)$. Therefore, every point in $B(x, r)$ is regular. So $d(x, \mathcal{S})>r$. This proves $(2.95)$ by the arbitrary choice of $x \in \mathcal{F}_{\tilde{K} r}$. Taking complement of (2.95), we obtain (2.96).

2.6. Compactness of $\widetilde{\mathscr{K} \mathscr{S}}(\boldsymbol{n}, \boldsymbol{\kappa})$. As a model space, $\widetilde{\mathscr{K} \mathscr{S}}(n, \kappa)$ should have compactness. However, we need first to obtain a weak compactness, then we improve regularity further to obtain the genuine compactness. It is not hard to see the weak-compactness theory of Anderson-Cheeger-Colding-Tian-Naber can be generalized to apply on $\widetilde{\mathscr{K} \mathscr{S}}(n, \kappa)$ without fundamental difficulties, almost verbatim. Actually, the key of Anderson-Cheeger-Colding-Tian-Naber theory is that one can approximate the distance function by harmonic function, or heat flow solution, which have much better regularity for developing integral estimates. These estimates are justified by the technical preparation in previous subsections.

Proposition 2.55 (Weak compactness). Suppose $\left(X_{i}, x_{i}, g_{i}\right) \in \widetilde{\mathscr{K} \mathscr{S}}(n, \kappa)$, by taking subsequences if necessary, we have

$$
\left(X_{i}, x_{i}, g_{i}\right) \stackrel{\hat{C}^{\infty}}{\longrightarrow}(\bar{X}, \bar{x}, \bar{g})
$$


for some length space $\bar{X}$ which satisfies all the properties of spaces in $\widetilde{\mathscr{K} \mathscr{S}}(n, \kappa)$ except the third and fourth properties, that is, the weak convexity of $\mathcal{R}$ and the Minkowski dimension estimate $\mathcal{S}$. The Hausdorff dimension of $\mathcal{S}$ is not greater than $2 n-4$.

Sketch of the proof. Note that each space in $\widetilde{\mathscr{K} \mathscr{S}}(n, \kappa)$ satisfies volume doubling property. Therefore, if there exists a sequence $\left(X_{i}, x_{i}, g_{i}\right) \in \widehat{\mathscr{K} \mathscr{S}}(n, \kappa)$, by standard ball-packing argument, it is clear that

$$
\left(X_{i}, x_{i}, g_{i}\right) \stackrel{G . H .}{\longrightarrow}(\bar{X}, \bar{x}, \bar{g})
$$

for some length space $\bar{X}$. Then let us list the properties satisfied by $\bar{X}$.

By Proposition 2.42, $\bar{X}$ inherits a natural measure from the limit process, which is a measure compatible with the limit metric structure, as that in [9]. Then the volume convergence follows, almost tautologically. It follows directly from this property and the volume comparison that $\bar{X}$ satisfies Property 6 in Definition 2.1 .

In the limit space $\bar{X}$, we can define regular points as the collection of points where every tangent space is $\mathbb{R}^{2 n}$, singular points as those points which are not regular. Let $\mathcal{R}(\bar{X})$ and $\mathcal{S}(\bar{X})$ be the regular and singular part of $\bar{X}$, respectively. We automatically obtain the regular-singular decomposition $\bar{X}=\mathcal{R}(\bar{X}) \cup \mathcal{S}(\bar{X})$. By a version of Anderson's gap theorem (see Proposition 2.46) and volume convergence, a blowup argument shows that each regular point has a small neighborhood which has a smooth manifold structure. Clearly, this manifold is Ricci-flat with a limit Kähler structure. So we proved Property 1 and Property 2, except the nonemptiness of $\mathcal{R}$.

For each point $x \in \bar{X}$, a tangent space is defined as the pointed-GromovHausdorff limit of $\left(\bar{X}, x, \lambda_{i}^{-1} d\right)$ for some sequence $\lambda_{i} \rightarrow 0$. Note that each tangent space of $\bar{X}$ is a volume cone, due to the volume convergence and Bishop-Gromov volume comparison, which can be established as that in [9]. Then it follows from Proposition 2.43 that every volume cone is actually a metric cone. Then an induction argument can be applied, like that in [9], to obtain the stratification of singularities $\mathcal{S}=\mathcal{S}_{1} \cup \mathcal{S}_{2} \cdots \cup \mathcal{S}_{2 n}$, where $\mathcal{S}_{k}$ is the union of singular points whose tangent space can split-off at least $(2 n-k)$-straight lines. In particular, generic points of $\bar{X}$ have tangent spaces $\mathbb{R}^{2 n}$. In other words, generic points are regular, so $\mathcal{R} \neq \emptyset$ and we finish the proof of Property 2 .

The Kähler condition guarantees that each tangent cone exactly splits off $\mathbb{C}^{k}$, by Proposition 2.44, as done in [10]. So the stratification of singular set can be improved as $\mathcal{S}=\mathcal{S}_{2} \cup \mathcal{S}_{4} \cup \ldots \mathcal{S}_{2 n}$. By Lemma 2.41, we can apply slice argument as that in [10] and [7]. Consequently, Chern-Simons theory (see [12]) implies that codimension 2 singularity cannot appear, due to the fact that a generic slice 
is a smooth surface with boundary, and the Ricci curvature's restriction on such a surface is zero. Actually, the smoothness of generic slices follows from the high codimension of the singular set (item 4 of Definition 2.1) and the gradient estimates of the harmonic approximation functions (Proposition 2.24). Therefore, $\mathcal{S}=\mathcal{S}_{4} \cup \ldots \mathcal{S}_{2 n}$, which means $\operatorname{dim}_{\mathcal{H}} \mathcal{S} \leqslant 2 n-4$.

Let $\bar{y} \in \mathcal{S}(\bar{X})$. Suppose $y_{i} \in X_{i}$ satisfies $y_{i} \rightarrow \bar{y}$. Then either there is a uniform $\xi$ such that every point in each $B\left(y_{i}, \xi\right)$ is regular (but without uniform curvature bound as $i$ increase), or we can choose $y_{i}$ such that every $y_{i}$ is singular. In the first case, we can use a blowup argument and Anderson's gap theorem to show that the volume density of $\bar{y}$ is strictly less than $1-2 \delta_{0}$. In the second case, we can use volume comparison and convergence to show $\mathrm{v}(\bar{y}) \leqslant 1-2 \delta_{0}$. So we proved Property 5.

We have checked all the properties of $\bar{X}$ as claimed. We now need to improve the convergence topology from Gromov-Hausdorff topology. However, this improvement follows from volume convergence and the improving regularity property of volume radius, Corollary 2.48 .

From the above argument, it is clear that no new idea is needed beyond the traditional theory, using the technical lemmas and propositions in the previous sections. Actually, weak compactness can be established under even weaker conditions, which will be discussed in our forthcoming work. Based on the weak compactness, we immediately obtain an $\epsilon$-regularity property, as that in [10].

Proposition 2.56 ( $\epsilon$-regularity). There exists an $\epsilon=\epsilon(n, \kappa)$ with the following properties.

Suppose $X \in \widetilde{\mathscr{K} \mathscr{S}}(n, \kappa), x_{0} \in X$. Suppose

$$
d_{G H}\left(B\left(x_{0}, 1\right), B\left(\left(z_{0}^{*}, 0\right), 1\right)\right)<\epsilon
$$

where $\left(z_{0}^{*}, 0\right) \in C\left(Z_{0}\right) \times \mathbb{R}^{2 n-3}$ for some metric cone $C\left(Z_{0}\right)$ with vertex $z_{0}^{*}$. Then we have

$$
\operatorname{vr}\left(x_{0}\right)>\frac{1}{2}
$$

Proof. Otherwise, there is a sequence of $\epsilon_{i} \rightarrow 0$ and $x_{i} \in X_{i}$ violating the statement. By weak compactness of $\widetilde{\mathscr{K} \mathscr{S}}(n, \kappa)$, we can assume $x_{i} \rightarrow x$ and $z_{i}^{*} \rightarrow z^{*}$ with the following identity holds.

$$
d_{G H}\left(\left(B(x, 1), B\left(\left(z^{*}, 0\right), 1\right)\right)\right)=0 .
$$

In particular, the tangent cone at $x$ is exactly the cone $C(Z) \times \mathbb{R}^{2 n-3}$, which must be $\mathbb{C}^{n}$ by the complex rigidity. Therefore, $B(x, 1)$ is the unit ball in $\mathbb{C}^{n}$. Thus, 
the volume convergence implies that for large $i, 2^{2 n}\left|B\left(x_{i}, \frac{1}{2}\right)\right|$ can be very close to 1 . In particular, $\operatorname{vr}\left(x_{i}\right)>\frac{1}{2}$ by the definition of volume radius. However, this contradicts our assumption.

Then we are able to move the integral estimate of [11] to $X \in \widetilde{\mathscr{K} \mathscr{S}}(n, \kappa)$.

Proposition 2.57 (Density estimate of regular points). For every $0<p<2$, there is a constant $E=E(n, \kappa, p)$ with the following properties.

Suppose $(X, x, g) \in \widehat{\mathscr{K} \mathscr{S}}(n, \kappa), r$ is a positive number. Then we have

$$
r^{2 p-2 n} \int_{B(x, r)} \operatorname{vr}(y)^{-2 p} d y \leqslant E(n, \kappa, p) .
$$

Proof. In light of Proposition 2.47, it is clear that volume radius and harmonic radius are uniformly equivalent. Therefore, Proposition 2.57 is nothing but a singular version of the Cheeger-Naber estimate (see the second inequality of part 2 of Corollary 1.26 in [11]). As pointed out by Cheeger and Naber, their estimate holds for Gromov-Hausdorff limit for Ricci-flat manifolds. Actually, going through their proof, it is clear that the smooth structure of the underlying space is not used. Intuitively, if Bishop-Gromov volume comparison holds, then most geodesic balls are almost volume cones, hence almost metric cones. However, if a cone is very close to a cone which splits off at least $(2 n-3)$-lines, then it must be Euclidean space by the $\epsilon$-regularity property. This intuition was quantified in [11], by the method they called quantitative calculus, which does not depends on smooth structure by its nature. We note that the quantitative calculus argument of [11] works when we have the following properties.

- Bishop-Gromov volume comparison, by Proposition 2.3.

- Weak compactness of $\widetilde{\mathscr{K} \mathscr{S}}(n, \kappa)$, by Proposition 2.55 .

- Volume convergence, by Proposition 2.42.

- Almost volume cone implies almost metric cone, by Proposition 2.43.

- $\epsilon$-regularity, by Proposition 2.56 .

Since all these properties hold on $\widetilde{\mathscr{K} \mathscr{S}}(n, \kappa)$, the proof follows that of [11] verbatim.

An immediate consequence of Proposition 2.57 is the following volume estimate of the neighborhood of the singular set. 
COROLlary 2.58 (Volume estimate of singularity neighborhood). Suppose ( $X$, $\left.x_{0}, g\right) \in \widetilde{\mathscr{K} \mathscr{S}}(n, \kappa), 0<\rho \ll 1$. Then for each $0<p<2$, we have

$$
\left|\left\{x \mid d(x, \mathcal{S})<\rho, x \in B\left(x_{0}, 1\right)\right\}\right|<C \rho^{2 p},
$$

for some $C=C(n, \kappa, p)$.

Proof. It follows from Definition 2.53 that

$$
\begin{aligned}
(2 r)^{-2 p}\left|B\left(x_{0}, 1\right) \cap \mathcal{F}_{r} \backslash \mathcal{F}_{2 r}\right| & =\int_{B\left(x_{0}, 1\right) \cap \mathcal{F}_{r} \backslash \mathcal{F}_{2 r}}(2 r)^{-2 p} \\
& <\int_{B\left(x_{0}, 1\right) \cap \mathcal{F}_{r} \backslash \mathcal{F}_{2 r}} \mathbf{v r}^{-2 p}<E(n, \kappa, p),
\end{aligned}
$$

which implies that

$$
\left|B\left(x_{0}, 1\right) \cap \mathcal{D}_{2 r} \backslash \mathcal{D}_{r}\right|<2^{2 p} E^{2 p} \Rightarrow\left|B\left(x_{0}, 1\right) \cap \mathcal{D}_{2 r}\right|<\frac{E}{1-4^{-p}}(2 r)^{2 p} .
$$

By virtue of (2.96), we have

$$
\begin{aligned}
\left|B\left(x_{0}, 1\right) \cap\{x \mid d(x, \mathcal{S})<\rho\}\right| & \leqslant\left|B\left(x_{0}, 1\right) \cap \mathcal{D}_{\tilde{K} \rho}\right| \\
& <\frac{E}{1-4^{-p}} \tilde{K}^{2 p} \rho^{2 p}<C \rho^{2 p} .
\end{aligned}
$$

Now we are ready to prove Theorem 1.3 , the compactness theorem.

Proof of Theorem 1.3. Suppose $\left(X_{i}, x_{i}, g_{i}\right) \in \widetilde{\mathscr{K} \mathscr{S}}(n, \kappa)$, we already know, by Proposition 2.55 , that $\left(X_{i}, x_{i}, g_{i}\right)$ converges to a limit space $(\bar{X}, \bar{x}, \bar{g})$, which satisfies almost all the properties of spaces in $\widetilde{\mathscr{K} \mathscr{S}}(n, \kappa)$, except the weak convexity of $\mathcal{R}$ and the Minkowski dimension estimate of $\mathcal{S}$. Fix every two points $\bar{y}, \bar{z} \in \mathcal{R} \subset \bar{X}$, we can find a sequence of points $y_{i}, z_{i} \in X_{i}$ such that $y_{i} \rightarrow \bar{y}$ and $z_{i} \rightarrow \bar{z}$. It is clear that $\operatorname{vr}\left(y_{i}\right) \rightarrow \operatorname{vr}(\bar{y})$ and $\operatorname{vr}\left(z_{i}\right) \rightarrow \operatorname{vr}(\bar{z})$. It follows from the global Harnack inequality of volume radius, Proposition 2.52, that each shortest geodesic $\gamma_{i}$ connecting $y_{i}$ and $z_{i}$ is uniformly regular. Consequently, the limit shortest geodesic $\bar{\gamma}$ connecting $\bar{y}$ and $\bar{z}$ is a smooth shortest geodesic. Therefore, we have actually proved that $\mathcal{R}$ is convex, rather than weakly convex. Furthermore, if we repeatedly use the first inequality in Proposition 2.52 and smooth convergence determined by volume radius, one can see that a shortest geodesic $\bar{\gamma}$ with smooth interior can be obtained, even if we drop the condition $\bar{y} \in \mathcal{R}$. In other words, if $\bar{z} \in \mathcal{R}, \bar{y} \in \bar{X}$, then there is a shortest geodesic $\bar{\gamma}$ connecting them, with smooth interior. This means that $\mathcal{R}$ is strongly convex. 
By convexity of $\mathcal{R}$, it is clear that the limit space $\bar{X}$ satisfies the BishopGromov volume comparison. By virtue of volume convergence and the same argument in Proposition 2.51, we see that vr is a continuous function under the pointed-Cheeger-Gromov topology. In other words, for every point $\bar{z} \in \bar{X}$, and points $z_{i} \in X_{i}$ satisfying $z_{i} \rightarrow \bar{z}$, we have $\operatorname{vr}(\bar{z})=\lim _{i \rightarrow \infty} \operatorname{vr}\left(z_{i}\right)$. For each $r>0$, by density estimate, Proposition 2.57, we see that inequality (2.97) holds for every $B\left(x_{i}, r\right)$ uniformly. Taking limit, by the convergence of volume radius, we obtain (2.97) holds on $(\bar{X}, \bar{x}, \bar{g})$, for each $p \in(1.5,2)$. Then it follows from Corollary 2.58 and the definition of Minkowski dimension (see Definition 2.2) that $\operatorname{dim}_{\mathcal{M}} \mathcal{S} \leqslant 2 n-4$.

Based on the compactness theorem, we can improve the regularity of each space in $\widetilde{\mathscr{K} \mathscr{S}}(n, \kappa)$.

Proof of Theorem 1.4. The strong convexity of $\mathcal{R}$ and $\operatorname{dim}_{\mathcal{M}} \mathcal{S} \leqslant 2 n-4$ follows from the argument in the proof of Theorem 1.3. Moreover, by Theorem 1.3, we know each tangent space, as a pointed-Gromov-Hausdorff limit, must locate in $\widetilde{\mathscr{K} \mathscr{S}}(n, \kappa)$. Since $Y$ is a volume cone, due to volume convergence, the splitting of $Y$ follows from Lemma 2.35 .

Theorems 1.3 and 1.4 inspire us to give the definition of conifold in Definition 1.2. Roughly speaking, a conifold is a space which is almost a manifold away from a small singular set, where every tangent space is a metric cone. Note that we abuse notation here since the conifold has different meaning in the literature of string theory (see [26]). It is easy to see that every Kähler orbifold with singularity codimension not less than 4 is a conifold in our sense. With this terminology, we see that $\widetilde{\mathscr{K} \mathscr{S}}(n, \kappa)$ is nothing but the collection of Calabi-Yau conifolds with Euclidean volume growth, that is,

$$
\lim _{r \rightarrow \infty} \frac{|B(x, r)|_{d \mu}}{\omega_{2 n} r^{2 n}} \geqslant \kappa, \quad \forall x \in X .
$$

Then Theorem 1.3 can be interpreted as that the moduli space of noncollapsed Calabi-Yau conifolds is compact, under the pointed-Cheeger-Gromov topology. Theorem 1.4 can be understood as that a 'weakly' Calabi-Yau conifold is really a conifold, due to an intrinsic improving regularity property originates from the intrinsic Ricci flatness of the underlying space. The property of the moduli space $\widetilde{\mathscr{K} \mathscr{S}}(n, \kappa)$ is quite clear now.

Proof of Theorem 1.1. It follows directly from Theorems 1.3, 1.4 and Definition 1.2 . 
Actually, along the route to prove Theorem 1.4, we shall be able to improve the regularity of the spaces in $\widetilde{\mathscr{K} \mathscr{S}}(n, \kappa)$ even further. For example, we believe the following statement is true.

Conjecture 2.59. At every point $x_{0}$ of a Calabi-Yau conifold $X \in \widetilde{\mathscr{K} \mathscr{S}}(n, \kappa)$, the tangent space is unique.

The above problem is only interesting when $n>2$ and away from generic singular point. Note that if $X$ is a limit space of a sequence of Ricci-flat manifolds, then the uniqueness of tangent cone is a well known open problem, in the classical theory of Cheeger-Colding-Tian. Clearly, similar questions can be asked for general Kähler-Einstein conifold. It is not hard to see that a compact KählerEinstein conifold is a projective variety. Due to its independent interest, we shall discuss this issue in another separate paper.

2.7. Space-time structure of $\widetilde{\mathscr{K} \mathscr{S}}(\boldsymbol{n})$. Every space $X \in \widetilde{\mathscr{K} \mathscr{S}}(n)$ can be regarded as a trivial Ricci flow solution. Therefore, Perelman's celebrated work [34] can be exploited to study $X$. Let us briefly recall some fundamental functionals defined for the Ricci flow by Perelman.

Suppose $\left\{\left(X^{m}, g(t)\right),-T \leqslant t \leqslant 0\right\}$ is a Ricci flow solution on a smooth complete Riemannian manifold $X$ of real dimension $m$. Suppose $x, y \in X$. Suppose $\gamma$ is a space-time curve parameterized by $\tau=-t$ such that

$$
\boldsymbol{\gamma}(0)=(x, 0), \quad \boldsymbol{\gamma}(\bar{\tau})=(y,-\bar{\tau}) .
$$

Let $\gamma$ be the space-projection curve of $\gamma$. In other words, we have

$$
\boldsymbol{\gamma}(\tau)=(\gamma(\tau),-\tau)
$$

By the way, for the simplicity of notations, we always use bold symbol of a Greek character to denote a space-time curve. The corresponding space projection will be denoted by the usual Greek character. Following Perelman, the Lagrangian of the space-time curve $\boldsymbol{\gamma}$ is defined as

$$
\mathcal{L}(\boldsymbol{\gamma})=\int_{0}^{\bar{\tau}} \sqrt{\tau}\left(R+|\dot{\gamma}|^{2}\right)_{g(-\tau)} d \tau .
$$

Among all such $\gamma$ 's that connected $(x, 0),(y,-\bar{\tau})$ and parameterized by $\tau$, there is at least one smooth curve $\boldsymbol{\alpha}$ which minimizes the Lagrangian. This curve is called a shortest reduced geodesic. The reduced distance between $(x, 0)$ and 
$(y,-\bar{\tau})$ is defined as

$$
l((x, 0),(y,-\bar{\tau}))=\frac{\mathcal{L}(\boldsymbol{\alpha})}{2 \sqrt{\bar{\tau}}} .
$$

Let $V=\dot{\alpha}$. Then $V$ satisfies the equation

$$
\nabla_{V} V+\frac{V}{2 \tau}+2 \operatorname{Ric}(V, \cdot)+\frac{\nabla R}{2}=0,
$$

which is called the reduced geodesic equation. It is easy to check that $\dot{\alpha}=V=\nabla l$. The reduced volume is defined as

$$
\mathcal{V}((x, 0), \bar{\tau})=\int_{X}(4 \pi \bar{\tau})^{-m / 2} e^{-l} d v .
$$

It is proved by Perelman that $(4 \pi \tau)^{-m / 2} e^{-l} d v$, the reduced volume element, is monotonically nonincreasing along each reduced geodesic emanating from $(x, 0)$.

Suppose the Ricci flow solution mentioned above is static, that is, Ric $\equiv 0$. Then it is easy to check that

$$
\left\{\begin{array}{l}
\mathcal{L}(\boldsymbol{\alpha})=\frac{d^{2}(x, y)}{2 \sqrt{\bar{\tau}}}, \\
l((x, 0),(y,-\bar{\tau}))=\frac{d^{2}(x, y)}{4 \bar{\tau}}, \\
\nabla_{V} V+\frac{V}{2 \tau}=0, \\
|\dot{\alpha}|^{2}=|V|^{2}=|\nabla l|^{2}=\tau l, \\
\mathcal{V}((x, 0), \bar{\tau})=\int_{X}(4 \pi \bar{\tau})^{-m / 2} e^{-d^{2} / 4 \bar{\tau}} d v .
\end{array}\right.
$$

Now we assume $X \in \widetilde{\mathscr{K} \mathscr{S}}(n)$. By a trivial extension in an extra time direction, we obtain a static, eternal singular Kähler-Ricci flow solution. Since distance structure is already known, we can define reduced distance, reduced volume, and so on, following the Equation (2.102). Clearly, this definition coincides with the original one when $X$ is smooth.

The following theorem is important to bridge the Cheeger-Colding's structure theory to the Ricci flow theory.

THEOREM 2.60 (Volume ratio and reduced volume). Suppose $X \in \widetilde{\mathscr{K} \mathscr{S}}(n), x \in$ $X$. Let $X \times(-\infty, 0]$ have the obvious static space-time structure. Then we have

$$
\begin{aligned}
\operatorname{avr}(X) & =\lim _{\tau \rightarrow \infty} \mathcal{V}((x, 0), \tau) . \\
\operatorname{v}(x) & =\lim _{\tau \rightarrow 0} \mathcal{V}((x, 0), \tau) .
\end{aligned}
$$


Proof. The proof relies on the volume cone structure at local tangent space, or tangent space at infinity. So the proofs of (2.103) and (2.104) are almost the same. For simplicity, we will only prove (2.103) and leave the proof for (2.104) to the readers.

Clearly, the real dimension of $X$ is $m=2 n$. For each $\epsilon$ small, we have

$$
m \omega_{m} \operatorname{avr}(X)+\epsilon>H^{-m+1}|\partial B(x, H)|>m \omega_{m} \operatorname{avr}(X)-\epsilon,
$$

whenever $H$ is large enough. Note that

$$
\begin{aligned}
\mathcal{V}\left((x, 0), H^{2}\right) & =(4 \pi)^{-m / 2} H^{-m} \int_{0}^{\infty}|\partial B(x, r)| e^{-r^{2} / 4 H^{2}} d r, \\
1 & =(4 \pi)^{-m / 2} H^{-m} \int_{0}^{\infty} m \omega_{m} r^{m-1} e^{-r^{2} / 4 H^{2}} d r .
\end{aligned}
$$

So we have

$$
\begin{aligned}
& \mathcal{V}\left((x, 0), H^{2}\right)-\operatorname{avr}(X) \\
& \quad=(4 \pi)^{-m / 2} H^{-m} \int_{0}^{\infty}\left\{|\partial B(x, r)|-m \omega_{m} \operatorname{avr}(X) r^{m-1}\right\} e^{-r^{2} / 4 H^{2}} d r .
\end{aligned}
$$

We can further decompose the last integral as follows.

$$
\begin{aligned}
& \left|\int_{0}^{\epsilon H}\left\{|\partial B(x, r)|-m \omega_{m} \operatorname{avr}(X) r^{m-1}\right\} e^{-r^{2} / 4 H^{2}} d r\right| \\
& \quad \leqslant m \omega_{m} \int_{0}^{\epsilon H} r^{m-1} e^{-r^{2} / 4 H^{2}} d r=m \omega_{m} H^{m} \int_{0}^{\epsilon} s^{m-1} e^{-s^{2} / 4} d s, \\
& \left|\int_{\epsilon H}^{\infty}\left\{|\partial B(x, r)|-m \omega_{m} \operatorname{avr}(X) r^{m-1}\right\} e^{-r^{2} / 4 H^{2}} d r\right| \\
& \quad \leqslant \epsilon \int_{\epsilon H}^{\infty} r^{m-1} e^{-r^{2} / 4 H^{2}} d r<\epsilon H^{m} \int_{0}^{\infty} e^{-s^{2} / 4} d s=\epsilon H^{m} \pi^{1 / 2} .
\end{aligned}
$$

Therefore, we have

$$
\left|\mathcal{V}\left((x, 0), H^{2}\right)-\operatorname{avr}(X)\right|<(4 \pi)^{-m / 2}\left\{m \omega_{m} \int_{0}^{\epsilon} s^{m-1} e^{-s^{2} / 4} d s+\epsilon \pi^{1 / 2}\right\} .
$$

Since the above inequality holds for every $H$ large enough, we see that

$$
\left|\lim _{\tau \rightarrow \infty} \mathcal{V}((x, 0), \tau)-\operatorname{avr}(X)\right|<(4 \pi)^{-m / 2}\left\{m \omega_{m} \int_{0}^{\epsilon} s^{m-1} e^{-s^{2} / 4} d s+\epsilon \pi^{1 / 2}\right\} .
$$

Let $\epsilon \rightarrow 0$, we obtain (2.103). 
Theorem 2.60 says that when we study the asymptotic behavior of $X$, the volume ratio and reduced volume play the same role. Note that volume ratio is monotone along radius direction on a manifold with nonnegative Ricci curvature. This property plays an essential role in Cheeger-Colding's theory. Since reduced volume is monotone along Ricci flow, Theorem 2.60 suggests that CheegerColding's theory can be transplanted to the Ricci flow case.

\section{Canonical radius}

In Section 2, we established the compactness of the model space $\widetilde{\mathscr{K} \mathscr{S}}(n, \kappa)$, following the route of Anderson-Cheeger-Colding-Tian-Naber. It is clear that the volume ratio's monotonicity is essential to this route. However, most Kähler manifolds do not have this monotonicity. For example, if we take out a time slice from a Kähler-Ricci flow solution, there is no obvious reason at all that volume ratio monotonicity holds on it. Therefore, in order to set up weak compactness for general Kähler manifolds, we have to give up the volume ratio monotonicity and search for a new route. This will be done in this section.

3.1. Motivation and definition. Let us continue the discussion in Section 2.6. As a consequence of the weak compactness of $\widetilde{\mathscr{K} \mathscr{S}}(n, \kappa)$, we have density estimate of volume radius, Proposition 2.57. For simplicity of notation, we fix some $p_{0}$ very close to 2 , say $p_{0}=2-(1 / 1000 n)$. Define

$$
\mathbf{E} \triangleq E\left(n, \kappa, p_{0}\right)+200 \omega_{2 n} \kappa^{-1} .
$$

Here we adjust the number $E\left(n, \kappa, p_{0}\right)$ to a much larger number, to reserve spaces for later use. Then Proposition 2.57 implies

$$
r^{2 p_{0}-2 n} \int_{B(x, r)} \operatorname{vr}(y)^{-2 p_{0}} d y<\mathbf{E} .
$$

The above inequality contains a lot of information. For example, it immediately implies that in every unit ball, there exists a fixed sized subball with uniform regularity.

Proposition 3.1 (Generic regular subball). Suppose $\left(X, x_{0}, g\right) \in \widetilde{\mathscr{K} \mathscr{S}}(n, \kappa)$, $r$ is a positive number. Then we have

$$
\mathcal{F}_{c_{b} r} \cap B\left(x_{0}, r\right) \neq \varnothing,
$$

where

$$
c_{b} \triangleq\left(\frac{\omega_{2 n} \kappa}{4 \mathbf{E}}\right)^{1 / 2 p_{0}}
$$


Proof. Let vr achieve maximum value at $y_{0}$ in the ball closure $\overline{B\left(x_{0}, r\right)}$. By inequality (3.2), we have

$$
\begin{aligned}
\operatorname{vr}\left(y_{0}\right)^{-2 p_{0}} & \leqslant f_{B(x, r)} \operatorname{vr}(y)^{-2 p_{0}} d y \leqslant\left(\omega_{2 n} \kappa\right)^{-1} r^{-2 n} \int_{B(x, r)} \operatorname{vr}(y)^{-2 p_{0}} d y \\
& \leqslant\left(\omega_{2 n} \kappa\right)^{-1} r^{-2 p_{0}} \mathbf{E} .
\end{aligned}
$$

It follows that

$$
\operatorname{vr}\left(y_{0}\right) \geqslant\left(\frac{\omega_{2 n} \kappa}{\mathbf{E}}\right)^{1 / 2 p_{0}} r>c_{b} r .
$$

By continuity of vr, there must exist a point $z \in B\left(x_{0}, r\right)$ such that $\operatorname{vr}(z)>c_{b} r$. In other words, we have $z \in \mathcal{F}_{c r} \cap B\left(x_{0}, r\right)$. So (3.3) holds.

Let $\mathbf{E}$ and $c_{b}$ be the constants defined in (3.1) and (3.4). Then we can choose a small constant $\epsilon_{b}$ such that

$$
\epsilon_{b} \triangleq \epsilon\left(n, \kappa, \frac{c_{b}}{100}\right)
$$

by the dependence in (2.90) of Proposition 2.52. Combining the estimates in $\widetilde{\mathscr{K} \mathscr{S}}(n, \kappa)$, we obtain Theorem 1.5. For the convenience of the readers, we copy it as follows.

ThEOREM 3.2 (A priori estimates in model spaces). Suppose $\left(X, x_{0}, g\right) \in$ $\widetilde{\mathscr{K} \mathscr{S}}(n, \kappa), r$ is a positive number. Then the following estimates hold.

(1) Strong volume ratio estimate: $\kappa \leqslant \omega_{2 n}^{-1} r^{-2 n}\left|B\left(x_{0}, r\right)\right| \leqslant 1$.

(2) Strong regularity estimate: $r^{2+k}\left|\nabla^{k} R m\right| \leqslant c_{a}^{-2}$ in the ball $B\left(x_{0}, c_{a} r\right)$ for every $0 \leqslant k \leqslant 5$ whenever $\mathbf{v r}\left(x_{0}\right) \geqslant r$.

(3) Strong density estimate: $r^{2 p_{0}-2 n} \int_{B\left(x_{0}, r\right)} \operatorname{vr}(y)^{-2 p_{0}} d y \leqslant \mathbf{E}$.

(4) Strong connectivity estimate: Every two points

$$
y_{1}, y_{2} \subset B\left(x_{0}, r\right) \cap \mathcal{F}_{(1 / 100) c_{b} r}(X)
$$

can be connected by a shortest geodesic $\gamma$ such that $\gamma \subset \mathcal{F}_{\epsilon_{b} r}(X)$.

Proof of Theorem 1.5, that is, Theorem 3.2. Clearly, the first property (strong volume ratio estimate) follows from the combination of the noncollapsing assumption, gap assumption (see items 5 and 6 of Definition 2.1) and the Bishop-Gromov volume comparison (see Proposition 2.3). The second property follows from Definition 2.45 and Corollary 2.48. The third property follows from Proposition 2.57 and definition equation (3.1). The fourth property follows from Proposition 2.52 and definition equations (3.5), (3.4) and (3.1). 
We shall show that a weak compactness of $\widetilde{\mathscr{K} \mathscr{S}}(n, \kappa)$ can be established using the estimates in Theorem 3.2, without knowing the volume ratio monotonicity. For this new route of weak-compactness theory, we define a scale called canonical radius with respect to $\widetilde{\mathscr{K} \mathscr{S}}(n, \kappa)$. Under the canonical radius, rough estimates like that in Theorem 3.2 are satisfied.

In this section, we focus on the study of smooth complete Kähler manifold. Every such a manifold is denoted by $\left(M^{n}, g, J\right)$, where $n$ is the complex dimension. The Hausdorff dimension, or real dimension of $M$ is $m=2 n$. We first need to make sense of the rough volume radius, without the volume ratio monotonicity.

DeFinition 3.3. Denote the set $\left\{r\left|0<r<\rho, \omega_{2 n}^{-1} r^{-2 n}\right| B\left(x_{0}, r\right) \mid \geqslant 1-\delta_{0}\right\}$ by $I_{x_{0}}^{(\rho)}$ where $x_{0} \in M, \rho$ is a positive number. Clearly, $I_{x_{0}}^{(\rho)} \neq \emptyset$ since $M$ is smooth. Define

$$
\mathbf{v r}^{(\rho)}\left(x_{0}\right) \triangleq \sup I_{x_{0}}^{(\rho)}
$$

For each pair $0<r \leqslant \rho$, define

$$
\begin{aligned}
& \mathcal{F}_{r}^{(\rho)}(M) \triangleq\left\{x \in M \mid \mathbf{v} \mathbf{r}^{(\rho)}(x) \geqslant r\right\}, \\
& \mathcal{D}_{r}^{(\rho)}(M) \triangleq\left\{x \in M \mid \mathbf{v r} \mathbf{r}^{(\rho)}(x)<r\right\} .
\end{aligned}
$$

DEFINITION 3.4. A subset $\Omega$ of $M$ is called $\epsilon$-regular-connected on the scale $\rho$ if every two points $x, y \in \Omega$ can be connected by a rectifiable curve $\gamma \subset \mathcal{F}_{\epsilon}^{(\rho)}$ and $|\gamma|<2 d(x, y)$. For notational simplicity, if the scale is clear in the context, we shall just say $\Omega$ is $\epsilon$-regular-connected.

Inspired by the estimates in Theorem 3.2, we can define the concept of canonical radius as follows.

DEFINITION 3.5. We say that the canonical radius (with respect to model space $\widetilde{\mathscr{K} \mathscr{S}}(n, \kappa))$ of a point $x_{0} \in M$ is not less than $r_{0}$ if for every $r<r_{0}$, we have the following properties.

(1) Volume ratio estimate: $\kappa \leqslant \omega_{2 n}^{-1} r^{-2 n}\left|B\left(x_{0}, r\right)\right| \leqslant \kappa^{-1}$.

(2) Regularity estimate: $r^{2+k}\left|\nabla^{k} R m\right| \leqslant 4 c_{a}^{-2}$ in the ball $B\left(x_{0}, \frac{1}{2} c_{a} r\right)$ for every $0 \leqslant k \leqslant 5$ whenever $\omega_{2 n}^{-1} r^{-2 n}\left|B\left(x_{0}, r\right)\right| \geqslant 1-\delta_{0}$.

(3) Density estimate: $r^{2 p_{0}-2 n} \int_{B\left(x_{0}, r\right)} \mathbf{v r ^ { ( r ) }}(y)^{-2 p_{0}} d y \leqslant 2 \mathbf{E}$.

(4) Connectivity estimate: $B\left(x_{0}, r\right) \cap \mathcal{F}_{\frac{1}{50} c_{b} r}^{(r)}(M)$ is $\frac{1}{2} \epsilon_{b} r$-regular-connected on the scale $r$. 
Then we define canonical radius of $x_{0}$ to be the supreme of all the $r_{0}$ with the properties mentioned above. We denote the canonical radius by $\mathbf{c r}\left(x_{0}\right)$. For subset $\Omega \subset M$, we define the canonical radius of $\Omega$ as the infimum of all $\operatorname{cr}(x)$ where $x \in \Omega$. We denote this canonical radius by $\operatorname{cr}(\Omega)$.

REMARK 3.6. In Definition 3.5, the first condition (volume ratio estimate) is used to guarantee the existence of Gromov-Hausdorff limit. The second condition (regularity estimate) is for the purpose of improving regularity. The third condition (density estimate), together with the second condition (regularity estimate), implies that the regular part is almost dense (see Theorem 3.18). The fourth condition (connectivity estimate) is defined to assure that the regular part is connected (see Proposition 3.12).

Because of the regularity estimate of Definition 3.5, it is useful to define the concept of canonical volume radius as follows.

DEFinition 3.7. Suppose $\rho_{0}=\boldsymbol{c r}\left(x_{0}\right)$. Then we define

$$
\operatorname{cvr}\left(x_{0}\right) \triangleq \mathbf{v r}^{\left(\rho_{0}\right)}\left(x_{0}\right) .
$$

We call $\operatorname{cvr}\left(x_{0}\right)$ the canonical volume radius of the point $x_{0}$.

REMARK 3.8. For every compact smooth manifold $M$, there is an $\eta>0$ such that every geodesic ball with radius less than $\eta$ must have normalized volume radius at least $1-\delta_{0}$. Then it is easy to see that $r^{2 p_{0}-2 n} \int_{B\left(x_{0}, r\right)} \mathbf{v} \mathbf{r}^{(r)}(y)^{-2 p_{0}} d y$ is a continuous function with respect to $x_{0}$ and $r$. Therefore, if $\rho_{0}=\operatorname{cr}\left(x_{0}\right)$ is a finite positive number, we have

$$
\rho_{0}^{2 p_{0}-2 n} \int_{B\left(x_{0}, \rho_{0}\right)} \mathbf{v r}^{\left(\rho_{0}\right)}(y)^{-2 p_{0}} d y \leqslant 2 \mathbf{E} .
$$

If $r \leqslant \mathbf{c r}(M)$, then $\mathbf{v r}{ }^{(r)} \leqslant \mathbf{c v r}$ as functions. Therefore, we have

$$
r^{2 p_{0}-2 n} \int_{B\left(x_{0}, r\right)} \operatorname{cvr}(y)^{-2 p_{0}} d y \leqslant r^{2 p_{0}-2 n} \int_{B\left(x_{0}, r\right)} \operatorname{vr}^{(r)}(y)^{-2 p_{0}} d y \leqslant 2 \mathbf{E} .
$$

Let $r_{0}$ be $\operatorname{cvr}\left(x_{0}\right)$. By Definition 3.7, it is clear that $r_{0} \leqslant \operatorname{cr}\left(x_{0}\right)$. If $r_{0}=$ $\operatorname{cvr}\left(x_{0}\right)<\operatorname{cr}\left(x_{0}\right)$, then we have

$$
\begin{aligned}
\omega_{2 n}^{-1} r_{0}^{-2 n}\left|B\left(x_{0}, r_{0}\right)\right| & =1-\delta_{0}, \\
\omega_{2 n}^{-1} r^{-2 n}\left|B\left(x_{0}, r\right)\right| & <1-\delta_{0}, \quad \forall r \in\left(r_{0}, \operatorname{cr}\left(x_{0}\right)\right) .
\end{aligned}
$$


If $r_{0}=\operatorname{cvr}\left(x_{0}\right)=\operatorname{cr}\left(x_{0}\right)$, then we only have

$$
\omega_{2 n}^{-1} r_{0}^{-2 n}\left|B\left(x_{0}, r_{0}\right)\right| \geqslant 1-\delta_{0} .
$$

It is possible that equality (3.9) does not hold on the scale $r_{0}$ in this case.

REMARK 3.9. The three radii functions, $\mathbf{c r}$, vr and cvr are all positive functions on the interior part of $M$. However, we do not know whether they are continuous in general.

We shall use canonical radius as a tool to study the weak-compactness theory of Kähler manifolds.

3.2. Rough estimates when canonical radius is bounded from below. We assume $\operatorname{cr}(M) \geqslant 1$ in the following discussion of this subsection. Under this condition, we collect important estimates for developing the weak compactness.

For simplicity of notation, we denote

$$
\mathcal{F}_{r} \triangleq \mathcal{F}_{r}^{(\mathbf{c r}(M))}, \quad \mathcal{D}_{r} \triangleq \mathcal{D}_{r}^{(\mathbf{c r}(M))}
$$

Note that this definition can be regarded as a generalization of the corresponding definition for metric spaces in $\widehat{\mathscr{K} \mathscr{S}}(n, \kappa)$. It coincides with the original one since $\operatorname{cr}(M)=\infty$ whenever $M \in \widetilde{\mathscr{K} \mathscr{S}}(n, \kappa)$.

Proposition 3.10. For every $0<r \leqslant \rho_{0} \leqslant 1, x_{0} \in M$, we have

$$
\begin{aligned}
& \left|B\left(x_{0}, \rho_{0}\right) \cap \mathcal{D}_{r}\right|<4 \mathbf{E} \rho_{0}^{2 n-2 p_{0}} r^{2 p_{0}}, \\
& \left|B\left(x_{0}, \rho_{0}\right) \cap \mathcal{F}_{r}\right|>\left(\kappa \omega_{2 n}-4 \mathbf{E} r^{2 p_{0}} \rho_{0}^{-2 p_{0}}\right) \rho_{0}^{2 n} .
\end{aligned}
$$

In particular, there exists at least one point $z \in B\left(x_{0}, \rho_{0}\right)$ such that

$$
\operatorname{cvr}(z)>c_{b} \rho_{0},
$$

where $c_{b}=\left(\kappa \omega_{2 n} / 4 \mathbf{E}\right)^{1 / 2 p_{0}}$.

Proof. Recall that $\mathbf{v r}^{(\mathbf{c r}(M))} \geqslant \mathbf{v} \mathbf{r}^{\left(\rho_{0}\right)}$. By density estimate (see Definition 3.5), we have

$$
\begin{aligned}
r^{-2 p_{0}}\left|B\left(x_{0}, \rho_{0}\right) \cap \mathcal{D}_{r}\right| & \leqslant \int_{B\left(x_{0}, \rho_{0}\right) \cap \mathcal{D}_{r}}\left\{\mathbf{v r}{ }^{(\mathbf{c r}(M))}\right\}^{-2 p_{0}} \leqslant \int_{B\left(x_{0}, \rho_{0}\right)}\left\{\mathbf{v r} \mathbf{r}^{\left(\rho_{0}\right)}\right\}^{-2 p_{0}} \\
& \leqslant 2 \mathbf{E} \rho_{0}^{2 n-2 p_{0}} .
\end{aligned}
$$


Then (3.13) follows from the above inequality. Recall that $\mathcal{D}_{r}$ is the set where $\mathbf{v} \mathbf{r}^{(\mathbf{c r}(M))}<r$. Together with the $\kappa$-noncollapsing condition, (3.13) yields (3.14). Let $r=c_{b} \rho_{0}$, then (3.13) implies

$$
\left|B\left(x_{0}, \rho_{0}\right) \cap \mathcal{F}_{c_{b} \rho_{0}}\right|>0 .
$$

In particular, $B\left(x_{0}, \rho_{0}\right) \cap \mathcal{F}_{c_{b} \rho_{0}} \neq \emptyset$. In other words, we can find a point $z \in B\left(x_{0}\right.$, $\rho_{0}$ ) satisfying $\mathbf{v r}^{(\mathbf{c r}(M))}>c_{b} \rho_{0}$ and consequently inequality (3.15).

Corollary 3.11. Suppose $x_{0} \in M, H \geqslant 1 \geqslant r$, then we have

$$
\left|B\left(x_{0}, H\right) \cap \mathcal{D}_{r}\right| \leqslant\left(\frac{2^{2 n+2}\left|B\left(x_{0}, 2 H\right)\right|}{\kappa \omega_{2 n}}\right) r^{2 p_{0}} \mathbf{E} .
$$

Proof. We try to fill the ball $B\left(x_{0}, H\right)$ with balls $B\left(y_{i}, \frac{1}{2}\right)$ such that $y_{i} \in B\left(x_{0}, H\right)$ until no more such balls can squeeze in. Clearly, we have

$$
B\left(x_{0}, H\right) \subset \bigcup_{i=1}^{N} B\left(y_{i}, 1\right), \quad \bigcup_{i=1}^{N} B\left(y_{i}, \frac{1}{2}\right) \subset B\left(x_{0}, H+\frac{1}{2}\right) \subset B\left(x_{0}, 2 H\right) .
$$

On one hand, the balls $B\left(y_{i}, \frac{1}{2}\right)$ are disjoint to each other. So we have

$$
N \kappa \omega_{2 n}\left(\frac{1}{2}\right)^{2 n} \leqslant \sum_{i=1}^{N}\left|B\left(y_{i}, 1\right)\right| \leqslant\left|B\left(x_{0}, 2 H\right)\right|, \quad \Rightarrow \quad N \leqslant \frac{2^{2 n}\left|B\left(x_{0}, 2 H\right)\right|}{\kappa \omega_{2 n}} .
$$

On the other hand, $B\left(x_{0}, H\right)$ is covered by $\bigcup_{i=1}^{N} B\left(y_{i}, 1\right)$. So we have

$$
\left|B\left(x_{0}, H\right) \cap \mathcal{D}_{r}\right| \leqslant \sum_{i=1}^{N}\left|B\left(y_{i}, 1\right) \cap \mathcal{D}_{r}\right| \leqslant 4 N \mathbf{E} r^{2 p_{0}} \leqslant\left(\frac{2^{2 n+2}\left|B\left(x_{0}, 2 H\right)\right|}{\kappa \omega_{2 n}}\right) r^{2 p_{0}} \mathbf{E},
$$

where we used (3.17) and (3.13).

Proposition 3.12. For every $r \leqslant 1$, two points $x, y \in \mathcal{F}_{r}$ can be connected by a curve $\gamma \subset \mathcal{F}_{\frac{1}{2} \epsilon_{b} r}$ with length $|\gamma|<3 d(x, y)$.

Proof. By rescaling if necessary, we can assume $r=1$. Then $\operatorname{cr}(M) \geqslant 1$.

Suppose $x, y \in \mathcal{F}_{1}$. If $d(x, y) \leqslant 1$, then there is a curve connecting $x, y$ and it satisfies the requirements, by the $\frac{1}{2} \epsilon_{b}$-regular connectivity property of the canonical radius. So we assume $H=d(x, y)>1$ without loss of generality. 
Let $\beta$ be a shortest geodesic connecting $x, y$ such that $\beta(0)=x$ and $\beta(H)=y$. Let $N$ be an integer locating in $[2 H, 2 H+1]$. Define

$$
s_{i}=\frac{H i}{N}, \quad x_{i}=\beta\left(s_{i}\right) .
$$

Clearly, $x_{0}=x, x_{N}=y$, which are both in $\mathcal{F}_{1} \subset \mathcal{F}_{c_{b} / 50}$. For each $1 \leqslant i \leqslant N-1$, $x_{i}$ may not locate in $\mathcal{F}_{c_{b} / 50}$. However, in the ball $B\left(x_{i}, \frac{1}{20}\right)$, there exists a point $x_{i}^{\prime}$ such that

$$
\operatorname{vr}\left(x_{i}^{\prime}\right) \geqslant \frac{1}{2} c_{b} \cdot \frac{1}{20}=\frac{c_{b}}{40}>\frac{c_{b}}{50} \text {. }
$$

Clearly, we have

$$
d\left(x_{i}^{\prime}, x_{i+1}^{\prime}\right) \leqslant d\left(x_{i}^{\prime}, x_{i}\right)+d\left(x_{i}, x_{i+1}\right)+d\left(x_{i+1}, x_{i+1}^{\prime}\right) \leqslant \frac{H}{N}+\frac{1}{10}<\frac{3}{5} \leqslant 1 .
$$

Since $\operatorname{cr}(M) \geqslant 1$, one can apply $\frac{1}{2} \epsilon_{b}$-regular connectivity property of the canonical radius to find a curve $\beta_{i}$ connecting $x_{i}^{\prime}$ and $x_{i+1}^{\prime}$ such that $\beta_{i} \subset \mathcal{F}_{(1 / 2) \epsilon_{b}}$. Moreover, we have

$$
\left|\beta_{i}\right| \leqslant 2 d\left(x_{i}^{\prime}, x_{i+1}^{\prime}\right) \leqslant 2\left(\frac{H}{N}+\frac{1}{10}\right) .
$$

Concatenating all $\beta_{i}$ 's, we obtain a curve $\gamma$ connecting $x=x_{0}, y=x_{N}$ and $\gamma \subset \mathcal{F}_{(1 / 2) \epsilon_{b}}$. Furthermore, we have

$$
\begin{aligned}
|\gamma| & =\sum_{i=0}^{N-1}\left|\beta_{i}\right| \leqslant 2 N\left(\frac{H}{N}+\frac{1}{10}\right)=2 H+\frac{1}{5} N \leqslant 2 H+\frac{2 H+1}{5} \leqslant \frac{12}{5} H+\frac{1}{5} \\
& <\frac{13}{5} H<3 H
\end{aligned}
$$

COROLlary 3.13. For every $x \in M, 0<r \leqslant 1$, if $\partial B(x, 2 r) \neq \emptyset$, we can find a curve $\gamma$ connecting $\partial B(x, r / 2)$ and $\partial B(x, r)$ such that

$$
\gamma \subset \mathcal{F}_{(1 / 2) \epsilon_{b} r}, \quad|\gamma| \leqslant 2 r .
$$

In particular, we have

$$
\partial B(x, r) \cap \mathcal{F}_{\left(\epsilon_{b} / 2\right) r} \neq \emptyset .
$$

Proof. Let $\beta$ be a shortest geodesic connecting $x$ and some point $y \in \partial B\left(x, \frac{9}{8} r\right)$. Let $z$ be the intersection point of $\beta$ and $\partial B\left(x, \frac{3}{8} r\right)$. Let $y^{\prime}, z^{\prime}$ be regular points around $y, z$, that is, we require

$$
y^{\prime} \in B\left(y, \frac{r}{8}\right) \cap \mathcal{F}_{\left(c_{b} / 8\right) r}, \quad z^{\prime} \in B\left(z, \frac{r}{8}\right) \cap \mathcal{F}_{c_{b} / 8 r} .
$$


Clearly, triangle inequality implies that

$$
d\left(y^{\prime}, z^{\prime}\right) \leqslant \frac{6}{8} r+\frac{1}{8} r+\frac{1}{8} r=r \leqslant 1 .
$$

Since $\operatorname{cr}(M) \geqslant 1$, by connectivity estimate, there is a curve $\alpha$ connecting $y^{\prime}$ and $z^{\prime}$ such that

$$
|\alpha| \leqslant 2 r, \quad \alpha \subset \mathcal{F}_{\epsilon_{b} / 2 r} .
$$

Note that $z^{\prime} \in B(x, r / 2)$ and $y^{\prime} \in B(x, r)^{c}$. The connectedness of $M$ guarantees that $\alpha$ must have intersection with both $\partial B(x, r / 2)$ and $\partial B(x, r)$. So we can truncate $\alpha$ to obtain a curve $\gamma$ which connects $\partial B(x, r / 2)$ and $\partial B(x, r)$. Clearly, we have

$$
\gamma \subset \alpha \subset \mathcal{F}_{\left(\epsilon_{b} / 2\right) r}, \quad|\gamma| \leqslant|\alpha| \leqslant 2 r .
$$

Proposition 3.14. Suppose $x \in M, 0<r \leqslant 1$. Then for every point $y \in$ $\mathcal{F}_{(1 / 2) \epsilon_{b} r} \cap \partial B(x, r)$. There is a curve $\gamma$ connecting $x$ and $y$ such that:

- $|\gamma|<10 r$;

- For each nonnegative integer $i, \gamma \cap B\left(x, 2^{-i} r\right) \backslash B\left(x, 2^{-i-1} r\right)$ contains a component which connects $\partial B\left(x, 2^{-i} r\right)$ and $\partial B\left(x, 2^{-i-1} r\right)$ and is contained in $\mathcal{F}_{2^{-i-3} \epsilon_{b}^{2} r}$.

Proof. Choose $y_{i}$ be a point on $\partial B\left(x, 2^{-i} r\right) \cap \mathcal{F}_{2^{-i-1} \epsilon_{b} r}$. By Proposition 3.12, for each $i \geqslant 0$, there is a curve $\gamma_{i}$ connecting $y_{i}$ and $y_{i+1}$ such that

$$
\left|\gamma_{i}\right|<9 \cdot 2^{-i-1} r, \quad \gamma_{i} \subset \mathcal{F}_{2^{-i-3} \epsilon_{b}^{2} r}
$$

Concatenating all the $\gamma_{i}$ 's to obtain $\gamma$. Then $\gamma$ satisfies all the properties.

For the purpose of improving regularity, we need to study the behavior of cvr. Similar to vr on spaces in $\widetilde{\mathscr{K} \mathscr{S}}(n, \kappa)$ (see Proposition 2.47), cvr satisfies a local Harnack inequality.

Proposition 3.15. There is a constant $K=K(n, \kappa)$ with the following properties.

Suppose $x \in X, r=\operatorname{cvr}(x)<1 / K$, then for every point $y \in B\left(x, K^{-1} r\right)$, we have

$$
\begin{aligned}
K^{-1} r & \leqslant \operatorname{cvr}(y) \leqslant K r, \\
\omega_{2 n}^{-1} \rho^{-2 n}|B(y, \rho)| & \geqslant 1-\frac{1}{100} \delta_{0}, \quad \forall \rho \in\left(0, K^{-1} r\right), \\
|R m|(y) & \leqslant K^{2} r^{-2}, \\
\operatorname{inj}(y) & \geqslant K^{-1} r .
\end{aligned}
$$


COROLlaRY 3.16. For every $r \in(0,1], \mathcal{F}_{r}(M)$ is a closed set. Moreover, cvr is an upper-semicontinuous function on $\mathcal{F}_{r}(M)$.

Proof. Fix $r \in(0,1]$. Suppose $x_{i} \in \mathcal{F}_{r}(M)$ converges to a point $x \in M$. Let $r_{i}=$ $\operatorname{cvr}\left(x_{i}\right)$. We need to show $\operatorname{cvr}(x) \geqslant r$. Clearly, this follows directly if $\lim _{i \rightarrow \infty} r_{i}=\infty$ by Proposition 3.15. Without loss of generality, we may assume $r_{i}$ is uniformly bounded from above. Using Proposition 3.15 again, we see that $r_{i}$ is uniformly bounded away from zero. Let $r$ be a limit of $r_{i}$. Then we have

$$
|B(x, r)|=\lim _{i \rightarrow \infty}\left|B\left(x_{i}, r_{i}\right)\right| \geqslant \lim _{i \rightarrow \infty}\left(1-\delta_{0}\right) \omega_{2 n} r_{i}^{2 n}=\left(1-\delta_{0}\right) \omega_{2 n} r^{2 n},
$$

which implies $\operatorname{cvr}(x) \geqslant r=\lim _{i \rightarrow \infty} r_{i}$ by definition of canonical volume radius and the fact that $r=\lim _{i \rightarrow \infty} r_{i} \leqslant 1 \leqslant$ cvr. Consequently, we have $x \in \mathcal{F}_{r}(M)$. Therefore, $\mathcal{F}_{r}(M)$ is a closed set by the arbitrary choice of $\left\{x_{i}\right\}$. From the above argument, we have already seen that

$$
\operatorname{cvr}(x) \geqslant \lim _{i \rightarrow \infty} \operatorname{cvr}\left(x_{i}\right)
$$

which means that cvr is an upper-continuous function on $\mathcal{F}_{r}(M)$.

Clearly, the conclusion in the above corollary is weaker than that in Proposition 2.51, since here we do not have a rigidity property like Proposition 2.50. However, even if $\operatorname{cvr}(x)>\lim _{i \rightarrow \infty} \operatorname{cvr}\left(x_{i}\right)$, the local Harnack inequality of $\mathbf{c v r}$ guarantees that $\operatorname{cvr}(x)<K \lim _{i \rightarrow \infty} \operatorname{cvr}\left(x_{i}\right)$. So $\operatorname{cvr}$ is better than general semicontinuous function. For example, in the decomposition $M=\mathcal{F}_{r} \cup \mathcal{D}_{r}$, every point $y \in \partial \mathcal{F}_{r}$ satisfies $r \leqslant \operatorname{cvr}(y) \leqslant K r$. In many situations, it is convenient to just regard $K=1$, that is, cvr being continuous, without affecting the effectiveness of the argument. Furthermore, up to perturbation, one can even regard cvr as smooth functions. Full details of the perturbation can be found in Appendix B.

\subsection{Kähler manifolds with canonical radius bounded from below. Similar} to the traditional theory, volume convergence is very important. However, in the current situation, the volume convergence can be proved in a much easier way.

Proposition 3.17 (Volume convergence). Suppose $\left(M_{i}, g_{i}, J_{i}\right)$ is a sequence of Kähler manifolds satisfying $\operatorname{cr}\left(M_{i}\right) \geqslant r_{0}$. Suppose $x_{i} \in M_{i}$ and $d_{i}$ is the length structure induced by $g_{i}$. Then we have

$$
\left(M_{i}, x_{i}, d_{i}\right) \stackrel{\text { P.G.H. }}{\longrightarrow}(\bar{M}, \bar{x}, \bar{d}) .
$$


Moreover, the volume (2n-dimensional Gromov-Hausdorff measure) is continuous under this convergence, that is, for every fixed $\rho_{0}>0$, we have

$$
\left|B\left(\bar{x}, \rho_{0}\right)\right|=\lim _{i \rightarrow \infty}\left|B\left(x_{i}, \rho_{0}\right)\right| .
$$

Proof. The existence of the Gromov-Hausdorff limit space follows from the volume doubling property and the standard ball-packing argument. Fix $r \ll \rho_{0}$, then it follows from the definition of $\mathcal{F}_{r}$ that the convergence on $B\left(x_{i}, \rho_{0}\right) \cap \mathcal{F}_{r}$ can be improved to $C^{4}$-topology. Then the volume converges trivially on this part. On the other hand, the volume of $B\left(x_{i}, \rho_{0}\right) \cap \mathcal{D}_{r}$ is bounded by $C r^{2 p_{0}}$, which tends to zero as $r \rightarrow 0$. So the volume convergence of geodesic balls $B\left(x_{i}, \rho_{0}\right)$ follows from the combination of the two factors mentioned above. More details are given as follows.

Let $(\bar{M}, \bar{x}, \bar{d})$ be the limit space. For each $r \leqslant r_{0}$, define

$\mathcal{R}_{r} \triangleq\left\{\bar{y} \in \bar{M} \mid\right.$ There exists $y_{i} \in M_{i}$ such that $y_{i} \rightarrow \bar{y}$ and $\left.\liminf _{i \rightarrow \infty} \operatorname{cvr}\left(y_{i}\right) \geqslant r\right\}$,

$$
\begin{gathered}
\mathcal{S}_{r} \triangleq\left(\mathcal{R}_{r}\right)^{c}, \\
\mathcal{R}^{\prime} \triangleq \bigcup_{0<r \leqslant r_{0}} \mathcal{R}_{r}, \\
\mathcal{S}^{\prime} \triangleq \bigcap_{0<r \leqslant r_{0}} \mathcal{S}_{r} .
\end{gathered}
$$

We now show that $\mathcal{S}^{\prime}$ is a subset of $\bar{M}$ of Minkowski dimension at most $2 n-2 p_{0}$. Without loss of generality, it suffices to show this dimension estimate for $\mathcal{S}^{\prime} \cap B\left(\bar{x}, \rho_{0}\right)$.

For each small $r>0$, we shall construct a covering for the set $\mathcal{S}_{r} \cap B\left(\bar{x}, \rho_{0}\right)$. Clearly, the choice $\bigcup_{z \in \mathcal{S}_{r} \cap B\left(\bar{x}, \rho_{0}\right)} B(z, r)$ is a cover, but with uncountably many balls. By Vitali covering lemma, we can find countable many $z_{k}$ 's such that $\bigcup_{z_{k}} B\left(z_{k}, r\right)$ is a disjoint union and

$$
\mathcal{S}_{r} \cap B\left(\bar{x}, \rho_{0}\right) \subset \bigcup_{z_{k}} B\left(z_{k}, 5 r\right)
$$

We shall show that this covering is actually a finite covering with number of balls $N$ uniformly bounded by $C r^{2 p_{0}-2 n}$. Let $z_{k}$ be the limit point of $z_{k, i} \in M_{i}$. For large $i$, it follows from the definition that $\operatorname{cvr}\left(z_{k, i}\right)<2 r$. By Proposition 3.15, we see that $B\left(z_{k, i}, 5 r\right) \subset \mathcal{D}_{5 K r}$. It follows that

$$
\bigcup_{k} B\left(z_{k, i}, 0.5 r\right) \subset \bigcup_{k} B\left(z_{k, i}, 5 r\right) \subset\left\{B\left(x_{i}, 2 \rho_{0}\right) \cap \mathcal{D}_{5 K r}\right\}
$$


Note that $\bigcup_{k} B\left(z_{k, i}, 0.5 r\right)$ is a disjoint union. Taking volume on the manifold $M_{i}$, using the volume ratio's lower bound and Proposition 3.10, we obtain

$$
N \kappa \omega_{2 n}(0.5 r)^{2 n} \leqslant \sum_{k}\left|B\left(z_{k, i}, 0.5 r\right)\right| \leqslant\left|B\left(x_{i}, 2 \rho_{0}\right) \cap \mathcal{D}_{5 K r}\right| \leqslant C(5 K r)^{2 p_{0}} .
$$

It follows that $N \leqslant C r^{2 p_{0}-2 n}$ for some uniform constant $C$. Therefore, the covering we choose in (3.29) is a finite covering with the number of balls dominated by $C r^{2 p_{0}-2 n}$. Since $\mathcal{S}^{\prime}$ is a subset of $\mathcal{S}_{r}$, we obtain a covering of $\mathcal{S}^{\prime} \cap$ $B\left(\bar{x}, \rho_{0}\right)$ by size- $r$ balls with number at most $C r^{2 p_{0}-2 n}$, where $C$ is independent of $r$. Therefore, we have

$$
\operatorname{dim}_{\mathcal{M}}\left\{\mathcal{S}^{\prime} \cap B\left(\bar{x}, \rho_{0}\right)\right\} \leqslant 2 n-2 p_{0} .
$$

In particular, $\mathcal{S}^{\prime} \cap B\left(\bar{x}, \rho_{0}\right)$ has $2 n$-Hausdorff measure zero, or volume zero. This means we can ignore the effect of $\mathcal{S}^{\prime}$ when we consider volume convergence. On the other hand, away from $\mathcal{S}^{\prime}$, the volume convergence is obvious. We therefore obtain the volume convergence property whenever $B\left(x_{i}, \rho_{0}\right)$ converges to $B\left(\bar{x}, \rho_{0}\right)$.

Now we are able to show the weak-compactness theorem.

THEOREM 3.18 (Rough weak compactness). We assume the same conditions as Proposition 3.17. Denote $\mathcal{R} \subset \bar{M}$ as the set of regular points, that is, the points with some small neighborhoods which have $C^{4}$-Riemannian manifolds structure. Denote $\mathcal{S} \subset \bar{M}$ as the set of singular points, that is, the points which are not regular. Then we have the regular-singular decomposition $\bar{M}=\mathcal{R} \cup \mathcal{S}$ with the following properties.

- The regular part $\mathcal{R}$ is an open, path connected $C^{4}$-Riemannian manifold. Furthermore, for every two points $x, y \in \mathcal{R}$, there exists a curve $\gamma$ connecting $x, y$ satisfying

$$
\gamma \subset \mathcal{R}, \quad|\gamma| \leqslant 3 d(x, y) .
$$

- The singular part $\mathcal{S}$ satisfies the Minkowski dimension estimate

$$
\operatorname{dim}_{\mathcal{M}} \mathcal{S} \leqslant 2 n-2 p_{0} .
$$

Proof. Let $(\bar{M}, \bar{x}, \bar{d})$ be the limit space. For each $r \leqslant r_{0}$, define $\mathcal{R}_{r}, \mathcal{S}_{r}$ as in (3.25) and (3.26). Define $\mathcal{R}^{\prime}, \mathcal{S}^{\prime}$ as in (3.27) and (3.28). Recall that the regular set $\mathcal{R} \subset \bar{M}$ is defined as the collection of points which have small neighborhoods with manifolds structure. We shall show that $\mathcal{R}^{\prime}$ is nothing but $\mathcal{R}$, that is, $\mathcal{R}=$ $\bigcup_{0<r \leqslant r_{0}} \mathcal{R}_{r}$. 
Actually, by regularity estimate property of canonical radius, for every fixed $r \in\left(0, r_{0}\right)$, every point $\bar{y} \in \mathcal{R}_{r}$, we see that the convergence to $B\left(\bar{y}, \frac{1}{3} c_{a} r\right)$ can be improved to be in the $C^{4}$-topology. Clearly, $B\left(\bar{y}, \frac{1}{3} c_{a} r\right)$ has a manifold structure. So $\mathcal{R}_{r} \subset \mathcal{R}$. Let $r \rightarrow 0$, we have $\bigcup_{0<r \leqslant r_{0}} \mathcal{R}_{r} \subset \mathcal{R}$. On the other hand, suppose $\bar{y} \in \mathcal{R}$. Then there is a ball $B(\bar{y}, r)$ with a manifold structure. By shrinking $r$ if necessary, we can assume that the volume ratio of this ball is very close to the Euclidean one. Note that the volume ( $2 n$-dimensional Hausdorff measure) converges when $\left(M_{i}, x_{i}, g_{i}\right)$ converges to $(\bar{M}, \bar{x}, \bar{g})$. Suppose $y_{i} \rightarrow \bar{y}, y_{i} \in M_{i}$. Then we have $\omega_{2 n}^{-1} r^{-2 n}\left|B\left(y_{i}, r\right)\right|>1-\delta_{0}$ for large $i$. By definition, this means that $\operatorname{cvr}\left(y_{i}, 0\right) \geqslant r$. It follows from the regularity estimates that $\bar{y} \in \mathcal{R}_{r} \subset \mathcal{R}^{\prime}$. By the arbitrary choice of $\bar{y}$, we obtain $\mathcal{R} \subset \mathcal{R}^{\prime}$. So we finish the proof of

$$
\mathcal{R}=\mathcal{R}^{\prime}=\bigcup_{0<r \leqslant r_{0}} \mathcal{R}_{r}
$$

Combining the above equation with the definitions in (3.27) and (3.28), we have $\mathcal{S}=\mathcal{S}^{\prime}$. Therefore, (3.32) follows from $\operatorname{dim}_{\mathcal{M}} \mathcal{S}^{\prime} \leqslant 2 n-2 p_{0}$, which can be proved following (3.30). Alternatively, we can prove (3.32) as follows.

Fix $r<r_{0}$. Let $\rho_{0}=r_{0}$ and take the limit of (3.13), we obtain

$$
\left|B\left(\bar{y}, r_{0}\right) \cap \mathcal{S}_{r}\right| \leqslant 4 \mathbf{E} r_{0}^{2 n-2 p_{0}} r^{2 p_{0}},
$$

for every $\bar{y} \in \bar{M}$. Suppose $y \in \mathcal{R}_{r} \subset \bar{M}$. The regularity estimate property of canonical radius yields that every point in $B\left(y, \frac{1}{4} c_{a} r\right)$ is regular. So $d(y, \mathcal{S}) \geqslant \frac{1}{4} c_{a} r$. It follows that

$$
\mathcal{R}_{r} \subset\left\{x \in \bar{M} \mid d(x, \mathcal{S}) \geqslant \frac{1}{4} c_{a} r\right\} \Leftrightarrow \mathcal{S}_{r} \supset\left\{x \in \bar{M} \mid d(x, \mathcal{S})<\frac{1}{4} c_{a} r\right\} .
$$

Therefore, we have

$$
\{x \in \bar{M} \mid d(x, \mathcal{S})<r\} \subset \mathcal{S}_{4 c_{a}^{-1} r},
$$

whenever $r$ is very small. Combining (3.33) and (3.34) yields

$$
\left|B\left(\bar{y}, r_{0}\right) \cap\{x \in \bar{M} \mid d(x, \mathcal{S})<r\}\right| \leqslant 4^{2 p_{0}+1} \mathbf{E} c_{a}^{-2 p_{0}} r_{0}^{2 n-2 p_{0}} r^{2 p_{0}}=C r^{2 p_{0}} .
$$

Since the above inequality holds for every small $r$ and every $\bar{y} \in \bar{M}$, it yields (3.32) directly.

It follows from the definition that $\mathcal{R}$ is an open $C^{4}$-manifold. The path connectedness of $\mathcal{R}$ follows from (3.31). Now we proceed to show (3.31). Fix $x, y \in \mathcal{R}$, let $r=\sup \left\{\rho \mid x \in \mathcal{R}_{\rho}, y \in \mathcal{R}_{\rho}\right\}$. Since $r>0$, we can choose sequence $x_{i}, y_{i} \in \mathcal{F}_{r / 2}\left(M_{i}\right)$ such that $x_{i} \rightarrow x, y_{i} \rightarrow y$. Let $\gamma_{i}$ be a curve connecting $x_{i}, y_{i}$ constructed by the method described in Proposition 3.12.Clearly, $\gamma_{i} \subset \mathcal{F}_{(1 / 4) \epsilon_{b} r}$ 
and $\left|\gamma_{i}\right|<3 d\left(x_{i}, y_{i}\right)$. Note that the convergence of $\mathcal{F}_{(1 / 4) \epsilon_{b} r}$ to its limit set is in $C^{4}$-topology. Consequently, the limit curve $\gamma$ satisfies (3.31).

Note that the convergence regularity was improved on $\mathcal{R}(\bar{M})$. Therefore, by abuse of notation, we now improve the convergence (3.24) as

$$
\left(M_{i}, x_{i}, g_{i}\right) \stackrel{\hat{C}^{4}}{\rightarrow}(\bar{M}, \bar{x}, \bar{g}),
$$

which means (3.24) together with the extra information that the convergence on $\mathcal{R}(\bar{M})$ happens in $C^{4}$-topology modulo diffeomorphisms. It is important to note that the length structure of $\bar{d}$ is not necessarily equivalent to the length structure induced by $\bar{g}$. Instead, only a rough equivalence (3.31) is known. Some other properties of the limit space $\bar{M}$ in Theorem 3.18 are also not good enough. For example, we do not know if every tangent space is a metric cone. In general, one should not expect these to hold. However, if $\left(M_{i}, g_{i}, J_{i}\right)$ is a blowup sequence from given Kähler-Ricci flow solutions with proper geometric bounds, we shall show that $\bar{M}$ does have much better properties. In particular, $\mathcal{R}$ is weakly geodesically convex and each tangent space is a metric cone. Such furthermore improvement will be discussed in a subsequent paper [17].

Proof of Theorem 1.6. It follows from the combination of the proof of Theorem 3.18 and the discussion in the above paragraph.

REMARK 3.19. The definition of regular points in Theorem 3.18 is stronger than the classical one, that is, a point is regular if and only if every tangent space at this point is isometric to $\mathbb{C}^{n}$. Therefore, some regular points in the classical definition may be singular in our definition.

REMARK 3.20. Note that in Definition 3.5, the definition of canonical radius, no Kähler condition is used. Therefore, the convergence results discussed in this subsection work naturally in the Riemannian setting.

REMARK 3.21. The canonical radius with respect to $\widetilde{\mathscr{K} \mathscr{S}}(n, \kappa)$ is reminiscent to the well known notion of harmonic radius (see Anderson [2]). For a sequence of Riemannian manifolds, once we control harmonic radius from below, we already control the local geometry (when coupled with elliptic equation such as Einstein equation) and we will obtain compactness theorem with respect to the smooth topology. However, canonical radius (with respect to $\widetilde{\mathscr{K} \mathscr{S}}(n, \kappa)$ ) is a weaker and more flexible notion than the harmonic radius. As Theorem 1.6 suggested, assuming lower bound of canonical radius only gives us a local geometry control away from some 'small' sets. 


\section{Acknowledgements}

This work is partially supported by National Science Foundation DMS1510401 and DMS-1515795. Both authors appreciate the anonymous referees for their careful reading. Their comments and suggestions are very useful for us to improve our presentation.

\section{Appendix A. Proof of weighted-Sobolev inequality}

The proof of Proposition 2.22 follows exactly the same strategy as [3, Proposition 2.1], which will be described below for the convenience of the readers.

Strategy of the proof of Proposition 2.22. Without loss of generality, we can assume $t=1$. Then the weighted-Sobolev inequality becomes

$$
\begin{aligned}
& \int_{X} f^{2}(x) p(t, x, y) d \mu_{x}-\left(\int_{X} f(x) p(t, x, y) d \mu_{x}\right)^{2} \\
& \quad \leqslant 2 \int_{X}|\nabla f|^{2}(x) p(t, x, y) d \mu_{x} .
\end{aligned}
$$

By a density argument, we can assume $f \in C_{c}^{\infty}(\mathcal{R})$ without loss of generality. Let $u$ be the heat solution initiating from $f$, that is, $u(y, 1)=\int_{X} f(x) p(1, x, y) d \mu_{x}$. Then we define

$$
\begin{aligned}
& \Psi_{0}(s)(y) \triangleq \int_{X} u^{2}(1-s, x) p(s, x, y) d \mu_{x}, \\
& \Psi(s)(y) \triangleq \int_{X}|\nabla u|^{2}(1-s, x) p(s, x, y) d \mu_{x} .
\end{aligned}
$$

According to this definition, we have

$$
\begin{aligned}
& \Psi_{0}(0)(y)=u^{2}(y, 1)=\left(\int_{X} f(x) p(1, x, y) d \mu_{x}\right)^{2}, \\
& \Psi_{0}(1)(y)=\int_{X} f^{2}(x) p(1, x, y) d \mu_{x}, \\
& \Psi(0)(y)=|\nabla u|^{2}(y, 1), \\
& \Psi(1)(y)=\int_{X}|\nabla f|^{2}(x) p(1, x, y) d \mu_{x} .
\end{aligned}
$$

Now the weighted-Sobolev inequality (A.1) is the same as

$$
\Psi_{0}(1)-\Psi_{0}(0) \leqslant 2 \Psi(1),
$$


which can be proved by the combination of the following three steps.

(1) $\dot{\Psi}_{0}(s)=2 \Psi(s)$ for each $s \in(0,1)$.

(2) $\Psi(a) \leqslant \Psi(b), \quad \forall 0<a<b<1$.

(3) $\lim _{s \rightarrow 1^{-}} \Psi(s)=\Psi(1)$.

Actually, since $\Psi_{0}$ is a continuous function on $[0,1]$, the above three steps yield that

$$
\Psi_{0}(1)-\Psi_{0}(0)=\int_{0}^{1} \dot{\Psi}_{0}(s) d s=\int_{0}^{1} 2 \Psi(s) d s \leqslant 2 \Psi(1) .
$$

Consequently, (A.1) is proved.

However, due to the existence of singularities, we need to check integrability and integration by parts very carefully in each step. The most delicate thing is to show that for a bounded heat solution $u$, we have $|\nabla u|^{2} \in N_{\text {loc }}^{1,2}(X)$ for positive time. This is trivial when $X$ is smooth and known when $\operatorname{dim}_{\mathcal{M}} \mathcal{S}<2 n-4$. We shall show that the same conclusion holds under the condition given by Definition 2.1. In fact, we first show that $\left.|\nabla| \nabla u\right|^{2} /|\nabla u|$ is locally integrable whenever $|\nabla u|>1$, which is proved in Lemma A.1. Then in Lemma A.2, by taking advantage of the weak convexity of $\mathcal{R}$, we show that actually $|\nabla| \nabla u \|^{2}$ is locally integrable whenever $|\nabla u|>1$.

LEMmA A.1. Suppose $u$ is a bounded heat solution, that is, $\square u=0$, on $X \times[0,1]$ satisfying

$$
|u|+|\dot{u}|+\int_{X}|\nabla \dot{u}|^{2}<K
$$

on $X \times\left[\frac{1}{2}, 1\right]$. At time $t=1$, let $w=\max \{|\nabla u|, 1\}$. Then we have

$$
\int_{B\left(x_{0}, 1\right) \backslash \mathcal{S}} \frac{|\nabla w|^{2}}{w}<H,
$$

where $H$ depends on $K$ and $n$, is independent of $x_{0}$.

Proof. Let $l=\dot{u}, v=|\nabla u|, m=2 n$. Direct calculation shows that

$$
\Delta v=\frac{|\nabla \nabla u|^{2}-|\nabla v|^{2}}{v}+\frac{\langle\nabla u, \nabla l\rangle}{v} \geqslant \frac{|\nabla \nabla u|^{2}-|\nabla v|^{2}}{v}-|\nabla l| .
$$

In local frame, we can delicately compare $|\nabla \nabla u|$ and $|\nabla v|$. Actually, by choosing normal coordinate such that $\nabla u=\partial / \partial x^{1}$, we then have $\Delta u=u_{11}+u_{22}+\cdots u_{m m}$ and $|\nabla v|^{2}=u_{11}^{2}+u_{12}^{2}+\cdots u_{1 m}^{2}$. Therefore, we have 


$$
\begin{aligned}
|\nabla \nabla u|^{2} & =u_{11}^{2}+u_{22}^{2}+\cdots+u_{m m}^{2}+2\left(u_{12}^{2}+\cdots+u_{1 m}^{2}\right) \\
& \geqslant u_{11}^{2}+\frac{\left(u_{22}+\cdots+u_{m m}\right)^{2}}{m-1}+2\left(u_{12}^{2}+\cdots+u_{1 m}^{2}\right) \\
& \geqslant u_{11}^{2}+\frac{\left(l-u_{11}\right)^{2}}{m-1}+2\left(u_{12}^{2}+\cdots+u_{1 m}^{2}\right) \\
& =\frac{m}{m-1} u_{11}^{2}+\frac{l^{2}-2 l u_{11}}{m-1}+2\left(u_{12}^{2}+\cdots+u_{1 m}^{2}\right) \\
& =\frac{m}{m-1}|\nabla v|^{2}+\frac{l^{2}-2 l u_{11}}{m-1}+\frac{m-2}{m-1}\left(u_{12}^{2}+\cdots+u_{1 m}^{2}\right)
\end{aligned}
$$

Then it is easy to see that

$$
\begin{aligned}
|\nabla \nabla u|^{2}-|\nabla v|^{2} & \geqslant \frac{1}{m-1}|\nabla v|^{2}+\frac{l^{2}-2 l u_{11}}{m-1} \geqslant \frac{1}{m-1}|\nabla v|^{2}-\frac{2 K}{m-1}|\nabla v| \\
& \geqslant \frac{3}{4(m-1)}|\nabla v|^{2}-\frac{4 K^{2}}{(m-1)} .
\end{aligned}
$$

Let $w=\max \{v, 1\}$, we claim that

$$
\Delta w \geqslant \frac{3}{4(m-1)} \frac{|\nabla w|^{2}}{w}-\frac{4 K^{2}}{(m-1)}-|\nabla l|,
$$

on $\mathcal{R}$ in the weak sense. Actually, let $\varphi \in C_{c}^{\infty}(\mathcal{R})$ and $\varphi \geqslant 0$. Let $\Omega$ be the domain consisting of points where $\varphi>0$ and $v>1$. Clearly, $\nabla w \equiv 0$ outside $\bar{\Omega}$. Following from the definition of weak Laplacian (Definition 2.16) and $\varphi$ has compact support, we have

$$
\begin{aligned}
-\int_{X} \varphi \Delta w & =\int_{X}\langle\nabla \varphi, \nabla w\rangle=\int_{\Omega}\langle\nabla \varphi, \nabla w\rangle=\int_{\Omega}\langle\nabla \varphi, \nabla v\rangle \\
& =-\int_{\Omega} \varphi \Delta v+\int_{\partial \Omega} \varphi\langle\nabla v, \vec{n}\rangle,
\end{aligned}
$$

where $\vec{n}$ is the outward normal vector field of $\Omega$ along $\partial \Omega$. Note that $\nabla w \equiv 0$ outside $\Omega$. So we have

$$
\begin{gathered}
\int_{X} \varphi \Delta w+\int_{X} \varphi\left(-\frac{3}{4(m-1)} \frac{|\nabla w|^{2}}{w}+\frac{4 K^{2}}{(m-1)}+|\nabla l|\right) \\
=\int_{\Omega} \varphi\left(\Delta v-\frac{3}{4(m-1)} \frac{|\nabla v|^{2}}{v}+\frac{4 K^{2}}{(m-1)}+|\nabla l|\right)
\end{gathered}
$$




$$
\begin{aligned}
& +\int_{X \backslash \Omega} \varphi\left(\frac{4 K^{2}}{(m-1)}+|\nabla l|\right)-\int_{\partial \Omega} \varphi\langle\nabla v, \vec{n}\rangle \\
\geqslant & -\int_{\partial \Omega} \varphi\langle\nabla v, \vec{n}\rangle \geqslant 0 .
\end{aligned}
$$

In the last step, $\vec{n}$ is the outward normal vector field along $\partial \Omega$ and $\varphi\langle\nabla v, \vec{n}\rangle \leqslant 0$. By the arbitrary choice of $\varphi$, the above inequality implies (A.3).

Suppose $\eta$ is a radial cutoff function which vanishes outside $B\left(x_{0}, 2\right)$ and equals 1 in $B\left(x_{0}, 1\right), \psi_{\epsilon}$ is a cutoff function which vanishes outside the $2 \epsilon$-neighborhood of $\mathcal{S}$ and equals 1 in the $\epsilon$-neighborhood of $\mathcal{S}$. We can also require that $|\nabla \eta|<2$, $\left|\nabla \psi_{\epsilon}\right|<2 / \epsilon$. Let $\chi=\eta\left(1-\psi_{\epsilon}\right)$. Multiplying both sides of the above inequality by $\chi^{2}$ and integrating by parts, we have

$$
\begin{aligned}
& \frac{1}{2(m-1)} \int_{X} \chi^{2} \frac{|\nabla w|^{2}}{w}+2(m-1) \int_{X} w|\nabla \chi|^{2} \\
& \geqslant-2 \int_{X}\langle\nabla \chi, \chi \nabla w\rangle=\int_{X} \chi^{2} \Delta w \\
& \geqslant \frac{3}{4(m-1)} \int_{X} \chi^{2} \frac{|\nabla w|^{2}}{w}-\frac{4 K^{2}}{m-1} \int_{X} \chi^{2}-\int_{X} \chi^{2}|\nabla l| .
\end{aligned}
$$

It follows that

$$
\begin{aligned}
& \frac{1}{4(m-1)} \int_{X} \chi^{2} \frac{|\nabla w|^{2}}{w} \\
& \leqslant C \int_{X} \chi^{2}(1+|\nabla l|)+2(m-1) \int_{X} w|\nabla \chi|^{2} \\
& \leqslant C \int_{X} \chi^{2}\left(1+|\nabla l|^{2}\right)+2(m-1) \int_{X} w|\nabla \chi|^{2} \\
& \leqslant C \int_{B\left(x_{0}, 2\right)}\left(1+|\nabla l|^{2}\right)+2(m-1) \int_{X}(1+|\nabla u|)|\nabla \chi|^{2} \\
& \leqslant C \int_{B\left(x_{0}, 2\right)}\left(1+|\nabla l|^{2}\right)+4(m-1) \int_{X}(1+|\nabla u|)\left\{|\nabla \eta|^{2}+\left|\nabla \psi_{\epsilon}\right|^{2}\right\} .
\end{aligned}
$$

In the support of $\psi_{\epsilon}, u$ satisfies heat equation from time $t=\frac{1}{2}$ to $t=1$. Moreover, $|u|$ is bounded by $K$. By adding $K$ if necessary, we can assume $u$ to be positive. In light of classical $\mathrm{Li}-\mathrm{Yau}$ gradient estimate for heat solutions (see [28, 33]), we see that $|\nabla u|<C(K) / \epsilon$ at time $t=1$. This can also be obtained from parabolic Moser iteration. Actually, let $y$ be a point such that $B(y, \epsilon)$ is regular. Since $\square|\nabla u| \leqslant 0$ in $B(y, \epsilon) \times[0,1]$ as smooth functions, Moser iteration implies that the value of $|\nabla u|(y, 1)$ is dominated by the $L^{2}$-average of $u$ 
in $B(y, \epsilon) \times\left[\frac{1}{2}, 1\right]$, multiplying by a number which is about $C / \epsilon$. Now we return to the main argument. Recall that $\left|\nabla \psi_{\epsilon}\right|<C / \epsilon$ also. The support of $\psi_{\epsilon}$ in $B\left(x_{0}, 2\right)$ has volume bounded above by $C \epsilon^{3+\delta}$ by the assumption $\operatorname{dim}_{\mathcal{M}} \mathcal{S}<2 n-3$. Consequently, we have

$$
\int_{B\left(x_{0}, 2\right)}|\nabla u|\left|\nabla \psi_{\epsilon}\right|^{2}<C \epsilon^{3+\delta} \cdot \epsilon^{-1} \cdot \epsilon^{-2}=C \epsilon^{\delta}=o(\epsilon) .
$$

In inequality (A.4), let $\epsilon \rightarrow 0$, we obtain $\int_{\mathcal{R}} \eta^{2}\left(|\nabla w|^{2} / w\right)<C$, which of course implies (A.2).

LEMMA A.2. Under the same conditions as in Lemma A.1. Then we have $w \in$ $N_{\text {loc }}^{1,2}(X)$.

Proof. It suffices to prove

$$
\int_{B\left(x_{0}, 1 / 2\right)}|\nabla w|^{2}<\infty
$$

for arbitrary point $x_{0} \in X$.

Let $\tilde{w}=\left(1-r^{2}\right)^{2} w, E=4 K^{2} /(m-1)$. It follows from (A.3) and the weak convexity of $\mathcal{R}$ (see Proposition 2.30) that

$$
\Delta w \geqslant \frac{1}{2(m-1)} \frac{|\nabla w|^{2}}{w}-E w-|\nabla l|, \quad \Delta\left(1-r^{2}\right)^{2} \geqslant-4 m .
$$

Then

$$
\begin{aligned}
\Delta \tilde{w}= & w \Delta\left(1-r^{2}\right)^{2}+2\left\langle\nabla\left(1-r^{2}\right)^{2}, \nabla w\right\rangle+\left(1-r^{2}\right)^{2} \Delta w \\
\geqslant & -4 m w-8\left(1-r^{2}\right) r\langle\nabla r, \nabla w\rangle+\frac{1}{2(m-1)}\left(1-r^{2}\right)^{2}|\nabla w|^{2} w^{-1} \\
& -E\left(1-r^{2}\right)^{2} w-\left(1-r^{2}\right)^{2}|\nabla l| \\
\geqslant & -4 m w-8\left(1-r^{2}\right)|\nabla w|+\frac{1}{2(m-1)}\left(1-r^{2}\right)^{2}|\nabla w|^{2} w^{-1} \\
& -E\left(1-r^{2}\right)^{2} w-\left(1-r^{2}\right)^{2}|\nabla l| \\
\geqslant & -C w-|\nabla l| .
\end{aligned}
$$

In short, we have $C w+|\nabla l| \geqslant-\Delta \tilde{w}$. Let $\tilde{w}_{k}=\min \{\tilde{w}, k\}$. Clearly, $\left.\tilde{w}_{k}\right|_{\partial \Omega}=0$ where $\Omega=B\left(x_{0}, 1\right)$. Let $\chi=1-\psi_{\epsilon}$. Multiplying both sides of the above inequality by $\chi^{2} \tilde{w}_{k}$, integration by parts implies that 


$$
\begin{aligned}
C & \int_{\Omega} \chi^{2} \tilde{w}_{k} w+\int_{\Omega} \chi^{2} \tilde{w}_{k}|\nabla l| \\
& \geqslant-\int_{\Omega} \chi^{2} \tilde{w}_{k} \Delta \tilde{w}=\int_{\Omega} \chi^{2}\left\langle\nabla \tilde{w}_{k}, \nabla \tilde{w}\right\rangle+2 \int_{\Omega}\left\langle\tilde{w}_{k} \nabla \chi, \chi \nabla \tilde{w}\right\rangle \\
& =\int_{\Omega} \chi^{2}\left|\nabla \tilde{w}_{k}\right|^{2}+2 \int_{\Omega}\left\langle\left(\tilde{w}_{k}-k\right) \nabla \chi, \chi \nabla \tilde{w}\right\rangle+2 k \int_{\Omega}\langle\nabla \chi, \chi \nabla \tilde{w}\rangle \\
& =\int_{\Omega} \chi^{2}\left|\nabla \tilde{w}_{k}\right|^{2}+2 \int_{\Omega}\left\langle\left(\tilde{w}_{k}-k\right) \nabla \chi, \chi \nabla \tilde{w}_{k}\right\rangle+2 k \int_{\Omega}\langle\nabla \chi, \chi \nabla \tilde{w}\rangle .
\end{aligned}
$$

Note that in the above inequality we used the fact that $\nabla \tilde{w}_{k}=0$ and $\tilde{w}_{k}-\tilde{w}=0$ whenever $w>k$. Applying an elementary inequality in the last step, we arrive

$$
\begin{aligned}
& C \int_{\Omega} \chi^{2} \tilde{w}_{k} w+\int_{\Omega} \chi^{2} \tilde{w}_{k}|\nabla l| \\
& \geqslant \frac{1}{2} \int_{\Omega} \chi^{2}\left|\nabla \tilde{w}_{k}\right|^{2}-2 \int_{\Omega}\left(\tilde{w}_{k}-k\right)^{2}|\nabla \chi|^{2} \\
&-2 k\left(\int_{\Omega} w|\nabla \chi|^{2}\right)^{1 / 2}\left(\int_{\Omega} \frac{\chi^{2}|\nabla \tilde{w}|^{2}}{w}\right)^{1 / 2} \\
& \geqslant \frac{1}{2} \int_{\Omega} \chi^{2}\left|\nabla \tilde{w}_{k}\right|^{2}-2 k^{2} \int_{\Omega}|\nabla \chi|^{2} \\
&-C k\left(\int_{\Omega} w|\nabla \chi|^{2}\right)^{1 / 2}\left(\int_{\Omega} \chi^{2}\left(w+\frac{|\nabla w|^{2}}{w}\right)\right)^{1 / 2} .
\end{aligned}
$$

Recall that $\chi=1-\psi_{\epsilon}$. Let $\epsilon \rightarrow 0$, the last two terms in the above inequality vanish. Then we have

$$
\begin{aligned}
\int_{\Omega}\left|\nabla \tilde{w}_{k}\right|^{2} & \leqslant C \int_{\Omega} \tilde{w}_{k}+\int_{\Omega} \tilde{w}_{k}|\nabla l| \leqslant C \int_{\Omega} w+\left(\int_{\Omega} \tilde{w}_{k}^{2}\right)^{1 / 2}\left(\int_{\Omega}|\nabla l|^{2}\right)^{1 / 2} \\
& \leqslant C \int_{\Omega}(1+|\nabla u|)+\left(\int_{\Omega} 1+|\nabla u|^{2}\right)^{1 / 2}\left(\int_{\Omega}|\nabla l|^{2}\right)^{1 / 2} \leqslant C .
\end{aligned}
$$

Note that the last constant $C$ does not depend on $k$. Let $k \rightarrow \infty$, we obtain $\int_{\Omega}|\nabla \tilde{w}|^{2} \leqslant C$. In particular, we can bound $\int_{B\left(x_{0}, 1 / 2\right)}|\nabla \tilde{w}|^{2}$ and consequently we have (A.5).

REMARK A.3. If $\operatorname{dim}_{\mathcal{M}} \mathcal{S}<2 n-4+(2 /(2 n-1))$, we can obtain Lemma A.2 without using the weak convexity of $\mathcal{R}$. The ingredient is to show subsolution property of $|\nabla u|^{q}$, for $q$ slightly bigger than $(2 n-2) /(2 n-1)$. Also, Lemmas A.1 and A.2 have versions for the bounded solution of Poisson equation $\Delta u=c$, where $c$ is a constant. 
After we know $w=\max \{1,|\nabla u|\} \in N_{\text {loc }}^{1,2}(X)$, parabolic De-Giorgi iteration implies that $w$ 's point wise bound can be dominated by $w$ 's $L^{2}$-norm in the spacetime. By Lemma A.2, we then have $w$ is bounded in Lemma A.4. This of course implies that $|\nabla u|$ is bounded.

Lemma A.4. Suppose $f \in C_{c}^{\infty}(\mathcal{R}), u$ is the heat solution initiating from $f$. Let $h=|\nabla u|^{2}(\cdot, t)$ for some $t>0$. Then $\|h\|_{L^{\infty}(X)}<\infty$.

Proof. Without loss of generality, we assume $t=1$.

Note that $\Delta u=\dot{u}$, which can be written down explicitly as

$$
\dot{u}(x, s)=\int_{X} f(y) \dot{p}(s, y, x) d \mu_{y} .
$$

By the exponential decay of $\dot{p}$ (see Proposition 2.20), it is clear that $\dot{u}$ also decays exponentially fast. Note that $\square \dot{u}=0$ on the regular part. Therefore, for each $s>0$, we have $\dot{u} \in L^{\infty}(X) \cap N^{1,2}(X)$. Let $l=\dot{u}, v=|\nabla u|, w=\max \{v, 1\}$. By Lemmas A.1 and A.2, we know $w(\cdot, s) \in N_{\text {loc }}^{1,2}(X)$ and $w \in N_{\text {loc }}^{1,2}(X \times(0, \infty))$. Moreover, we have

$$
\dot{v}=\partial_{t}(|\nabla u|)=\frac{\langle\nabla u, \nabla \dot{u}\rangle}{v}, \quad|\dot{v}| \leqslant|\nabla \dot{u}| .
$$

For each $0<t_{1}<s<t_{2}<\infty$, it is clear that $\dot{v}(\cdot, s) \in L^{2}(\mathcal{R})$ and

$$
\int_{t_{1}}^{t_{2}}\|\dot{v}\|_{L^{2}(\mathcal{R})}^{2} d s \leqslant \int_{t_{1}}^{t_{2}}\|\dot{u}\|_{N^{1,2}(\mathcal{R})}^{2} d s<\infty .
$$

Direct calculation shows that

$$
\square v=\left(\partial_{s}-\Delta\right) v=\frac{-|\nabla \nabla u|^{2}+\left.|\nabla| \nabla u\right|^{2}}{v} \leqslant 0 .
$$

Recall that $w=\max \{v, 1\}$. So we have $\square w \leqslant 0$ on $\mathcal{R} \times(0, \infty)$ in the weak sense, that is, for each nonnegative smooth cutoff function $\varphi$ compactly supported on $\mathcal{R} \times(0, \infty)$, we have

$$
\iint_{\mathcal{R} \times(0, \infty)} \varphi \square w \triangleq \iint_{\mathcal{R} \times(0, \infty)}\{\dot{w} \varphi+\langle\nabla \varphi, \nabla w\rangle\} \geqslant 0 .
$$

This can be proved following similar argument as that in the proof of inequality (A.3) in Lemma A.1. Since $w \in N_{\text {loc }}^{1,2}(X \times(0, \infty)),\|\dot{w}(\cdot, s)\|_{L^{2}(X)}^{2}$ is locally integrable on $(0, \infty)$, the compactly supported smooth functions are dense in $N^{1,2}(X \times(0, \infty))$, we have $\square w \leqslant 0$ in the weak sense on $X \times(0, \infty)$. 
By parabolic version of De-Giorgi iteration, we then have $w$ is locally bounded. Consequently, $|\nabla u|$ is locally bounded. Similar to subharmonic extension theorem (see Proposition 2.19), we have a heat subsolution extension theorem. Since $|\nabla u|$ is locally bounded and it is a heat subsolution on $\mathcal{R} \times(0, \infty)$, we obtain that $|\nabla u|$ is a heat subsolution on $X \times(0, \infty)$. In particular, we have $|\nabla u| \in N_{\text {loc }}^{1,2}(X \times(0, \infty))$. Therefore, by parabolic De-Giorgi iteration again, $\|\nabla u\|_{L^{\infty}\left(B(x, 1) \times\left[\frac{3}{4}, \frac{5}{4}\right]\right)}$ is bounded by $\|\nabla u\|_{L^{2}\left(B(x, 2) \times\left[\frac{1}{2}, 2\right]\right)}$, which is uniformly bounded, independent of the choice of $x$. Therefore, $|\nabla u|(\cdot, 1)$, and hence $h$, are globally bounded on $X$.

We continue to show the integrability of $\Delta|\nabla u|^{2}$ and $\partial_{t}|\nabla u|^{2}$.

Lemma A.5. Same conditions as that in Lemma A.4. Then $h \in N^{1,2}(X)$ and $|\Delta h|+|\dot{h}| \in L^{1}(X)$.

Proof. Let us first show that $|\dot{h}| \in L^{1}(X)$. Note that $\dot{u}=\Delta u$ is also a bounded heat solution, due to the exponential decay of $\dot{p}$. Since both $u$ and $\Delta u$ decay exponentially fast at infinity, we see that $u, \Delta u \in N^{1,2}(X)$. Therefore, we have

$$
\int_{X}|\dot{h}|=2 \int_{X}|\langle\nabla \Delta u, \nabla u\rangle|<C\left(\int_{X}|\nabla u|^{2}\right)^{1 / 2}\left(\int_{X}|\nabla \Delta u|^{2}\right)^{1 / 2}<\infty .
$$

This means that $|\dot{h}| \in L^{1}(X)$.

Then we continue to show that $|\Delta h| \in L^{1}(X)$. Actually, we have

$$
\Delta h=\Delta|\nabla u|^{2}=2|\nabla \nabla u|^{2}+2\langle\nabla u, \nabla \dot{u}\rangle .
$$

Fix $x_{0} \in X$. Let $\eta_{k}$ be a radial cutoff function which vanishes outside $B\left(x_{0}, k+1\right)$ and equals 1 in $B\left(x_{0}, k\right),\left|\nabla \eta_{k}\right|<2$. Let $\psi_{\epsilon}$ be as usual. Let $\chi_{k}=\eta_{k}\left(1-\psi_{\epsilon}\right)$. Then we have

$$
\begin{aligned}
& \int_{X} \chi_{k}^{2}|\nabla \nabla u|^{2}+\int_{X} \chi_{k}^{2}\langle\nabla u, \nabla \dot{u}\rangle \\
& \quad=\frac{1}{2} \int_{X} \chi_{k}^{2} \Delta|\nabla u|^{2}=-\frac{1}{2} \int_{X}\left\langle\nabla|\nabla u|^{2}, \nabla \chi_{k}^{2}\right\rangle=-2 \int_{X}\left\langle\chi_{k} \nabla|\nabla u|,|\nabla u| \nabla \chi_{k}\right\rangle \\
& \quad \leqslant\left.\frac{1}{2} \int_{X} \chi_{k}^{2}|\nabla| \nabla u\right|^{2}+2 \int_{X}|\nabla u|^{2}\left|\nabla \chi_{k}\right|^{2} \\
& \quad \leqslant \frac{1}{2} \int_{X} \chi_{k}^{2}|\nabla \nabla u|^{2}+2 \int_{X}|\nabla u|^{2}\left|\nabla \chi_{k}\right|^{2} .
\end{aligned}
$$

Recall that $\left|\nabla \chi_{k}\right|^{2} \leqslant 2\left(\left|\nabla \eta_{k}\right|^{2}+\left|\nabla \psi_{\epsilon}\right|^{2}\right)$ and $|\dot{h}|=2|\langle\nabla u, \nabla \dot{u}\rangle|$. Then we have

$$
\int_{X} \chi_{k}^{2}|\nabla \nabla u|^{2} \leqslant 8 \int_{B\left(x_{0}, k+1\right)}|\nabla u|^{2}\left(\left|\nabla \eta_{k}\right|^{2}+\left|\nabla \psi_{\epsilon}\right|^{2}\right)+\int_{X}|\dot{h}| .
$$


Note that $|\nabla u|$ is bounded here, due to Lemma A.4. Let $\epsilon \rightarrow 0$, we have

$$
\int_{B\left(x_{0}, k\right)}|\nabla \nabla u|^{2} \leqslant \int_{X} \eta_{k}^{2}|\nabla \nabla u|^{2} \leqslant 32 \int_{B\left(x_{0}, k+1\right)}|\nabla u|^{2}+\int_{X}|\dot{h}| .
$$

Let $k \rightarrow \infty$, by (A.6), we obtain

$$
\int_{X}|\nabla \nabla u|^{2} \leqslant 32 \int_{X}|\nabla u|^{2}+\int_{X}|\dot{h}|<C .
$$

It follows that

$$
\int_{X}|\Delta h| \leqslant 2 \int_{X}|\nabla \nabla u|^{2}+\int_{X}|\dot{h}|<\infty .
$$

So we proved that $|\Delta h| \in L^{1}(X)$ and hence $|\dot{h}|+|\Delta h| \in L^{1}(X)$.

Finally, we show that $h \in N^{1,2}(X)$. Recall that $h=|\nabla u|^{2}$ is bounded. So we have

$$
\begin{aligned}
\int_{X}\left(h^{2}+|\nabla h|^{2}\right) & =\int_{X}\left(|\nabla u|^{4}+\left.4|\nabla u|^{2}|\nabla| \nabla u\right|^{2}\right) \\
& \leqslant C \int_{X}\left(|\nabla u|^{2}+\left.|\nabla| \nabla u\right|^{2}\right) \leqslant C\left(1+\int_{X}|\nabla \nabla u|^{2}\right) .
\end{aligned}
$$

Plugging (A.7) into the above inequality, we have $h \in N^{1,2}(X)$.

After we obtain that $|\nabla u|^{2} \in N^{1,2}(X)$, in the following Lemmas A.6-A.8, we focus on the checking of integration by parts and continuity of integrals at boundary time. The heat kernel's exponential decay will play an important role there. However, the following proof will be by no means optimal. We only prove what we need by what we have.

Lemma A.6. Same conditions as that in Lemma A.4. Suppose $x_{0} \in X$. Then we have

$$
\int_{\mathcal{R}} h \Delta p\left(t, \cdot, x_{0}\right)=\int_{\mathcal{R}} p\left(t, \cdot, x_{0}\right) \Delta h .
$$

Proof. For simplicity of notation, denote $p\left(t, \cdot, x_{0}\right)$ by $p$.

We first note that both sides of (A.8) are finite integral. Actually, we have

$$
\begin{aligned}
& \left|\int_{\mathcal{R}} h \Delta p\right| \leqslant\left|\int_{\mathcal{R}}\right| h|| \Delta p|| \leqslant\|h\|_{L^{2}(X)}\|\Delta p\|_{L^{2}(\mathcal{R})}<\infty, \\
& \left|\int_{\mathcal{R}} p \Delta h\right| \leqslant\left|\int_{\mathcal{R}}\right| p\|\Delta h|| \leqslant\| p\left\|_{L^{\infty}(X)}\right\| \Delta h \|_{L^{1}(\mathcal{R})}<\infty .
\end{aligned}
$$


Then we show that both sides of (A.8) can be approximated by integrations over compact supported sets. Let $\eta_{k}$ be a radial cutoff function which vanishes outside $B\left(x_{0}, k+1\right)$ and equals 1 in $B\left(x_{0}, k\right),\left|\nabla \eta_{k}\right|<2$. Then we have

$$
\begin{aligned}
\left|\int_{\mathcal{R}} h \Delta p-\int_{\mathcal{R}} \eta_{k}^{2} h \Delta p\right| & =\left|\int_{\mathcal{R} \backslash B\left(x_{0}, k\right)}\left(1-\eta_{k}^{2}\right) h \Delta p\right| \leqslant\left|\int_{\mathcal{R} \backslash B\left(x_{0}, k\right)}\right| h|| \Delta p|| \\
& \leqslant\|\Delta p\|_{L^{2}\left(X \backslash B\left(x_{0}, k\right)\right)}\|h\|_{L^{2}(X)} .
\end{aligned}
$$

Clearly, $\|\Delta p\|_{L^{2}\left(X \backslash B\left(x_{0}, k\right)\right)} \rightarrow 0$ as $k \rightarrow \infty$, due to the exponential decay of $\Delta p$. Note that $h \in L^{2}(X)$ by Lemma A.5. Thus we have proved that

$$
\lim _{k \rightarrow \infty} \int_{\mathcal{R}} \eta_{k}^{2} h \Delta p=\int_{\mathcal{R}} h \Delta p .
$$

Similarly, we calculate

$$
\begin{aligned}
\left|\int_{\mathcal{R}} p \Delta h-\int_{\mathcal{R}} \eta_{k}^{2} p \Delta h\right| & =\left|\int_{\mathcal{R} \backslash B\left(x_{0}, k\right)}\left(1-\eta_{k}^{2}\right) p \Delta h\right| \leqslant\left|\int_{\mathcal{R} \backslash B\left(x_{0}, k\right)}\right| p \| \Delta h|| \\
& \leqslant\|p\|_{L^{\infty}\left(X \backslash B\left(x_{0}, k\right)\right)}\|\Delta h\|_{L^{1}(X)} .
\end{aligned}
$$

It follows from the exponential decay that $\|p\|_{L^{\infty}\left(X \backslash B\left(x_{0}, k\right)\right)} \rightarrow 0$ as $k \rightarrow \infty$. Also, we know $\Delta h \in L^{1}(X)$ by Lemma A.5. So we have

$$
\lim _{k \rightarrow \infty} \int_{\mathcal{R}} \eta_{k}^{2} p \Delta h=\int_{\mathcal{R}} p \Delta h .
$$

Clearly, $\eta_{k}^{2} h \in N_{c}^{1,2}(X)$ and $p \in N_{c}^{1,2}(X)$, both of them are bounded functions. Furthermore, both $|\Delta p|$ and $|\Delta h|$ are integrable on $B\left(x_{0}, k+1\right)$. Due to the fact that Minkowski codimension of $\mathcal{S}$ is greater than 2, it is not hard to check that

$$
-\int_{\mathcal{R}}\left\langle\nabla\left(\eta_{k}^{2} h\right), \nabla p\right\rangle=\int_{\mathcal{R}} \eta_{k}^{2} h \Delta p, \quad-\int_{\mathcal{R}}\left\langle\nabla\left(\eta_{k}^{2} p\right), \nabla h\right\rangle=\int_{\mathcal{R}} \eta_{k}^{2} p \Delta h .
$$

It follows that

$$
\int_{\mathcal{R}} \eta_{k}^{2} h \Delta p-\int_{\mathcal{R}} \eta_{k}^{2} p \Delta h=\int_{\mathcal{R}} 2 \eta_{k}\left\langle\nabla \eta_{k}, p \nabla h-h \nabla p\right\rangle .
$$

Denoting $B\left(x_{0}, k+1\right) \backslash B\left(x_{0}, k\right)$ by $A_{k}$, we have

$$
\begin{aligned}
& \left|\int_{\mathcal{R}} \eta_{k}^{2} h \Delta p-\int_{\mathcal{R}} \eta_{k}^{2} p \Delta h\right| \\
& \quad=\left|\int_{\mathcal{R}} 2 \eta_{k}\left\langle\nabla \eta_{k}, p \nabla h-h \nabla p\right\rangle\right| \leqslant 4 \int_{B\left(x_{0}, k+1\right) \backslash B\left(x_{0}, k\right)}|p \nabla h-h \nabla p|
\end{aligned}
$$




$$
\begin{aligned}
& \leqslant 4 \int_{A_{k}}|p \nabla h|+|h \nabla p| \\
& \leqslant 4\left(\int_{A_{k}} p^{2}\right)^{\frac{1}{2}}\left(\int_{A_{k}}|\nabla h|^{2}\right)^{1 / 2}+4\left(\int_{A_{k}} h^{2}\right)^{1 / 2}\left(\int_{A_{k}}|\nabla p|^{2}\right)^{1 / 2} \\
& \leqslant 8\|p\|_{N^{1,2}\left(A_{k}\right)}\|h\|_{N^{1,2}(X)} .
\end{aligned}
$$

By the exponential decay of $p$ and $\Delta p$, it is clear that $\|p\|_{N^{1,2}\left(A_{k}\right)} \rightarrow 0$ as $k \rightarrow \infty$. Therefore, we have

$$
\lim _{k \rightarrow \infty} \int_{\mathcal{R}} \eta_{k}^{2} f \Delta p=\lim _{k \rightarrow \infty} \int_{\mathcal{R}} \eta_{k}^{2} p \Delta h .
$$

Therefore, (A.8) follows from the combination of (A.9), (A.10) and (A.11).

Lemma A.7. Under the same conditions as that in Lemma A.4. Fix $T>0$, then for every pair $0<a<b<T$, we have

$$
\begin{gathered}
\int_{X} h(x, T-b) p(b, x, y) d \mu_{x}-\int_{X} h(x, T-a) p(a, x, y) d \mu_{x} \\
=2 \int_{a}^{b} \int_{\mathcal{R}} p\left(t, x, x_{0}\right)|\nabla \nabla u|^{2}(x) d \mu_{x} d t .
\end{gathered}
$$

Proof. Applying the Hölder inequality, we see that each integral on the left hand side of (A.12) is well defined and finite. Direct calculation shows that

$$
\begin{aligned}
& \frac{d}{d t} \int_{X} h(x, T-t) p(t, x, y) d \mu_{x} \\
& \quad=\int_{X}-\dot{h} p+h \dot{p}=\int_{\mathcal{R}}-\dot{h} p+h \Delta p=\int_{\mathcal{R}}(-\dot{h}+\Delta h) p .
\end{aligned}
$$

Note that we have used the integrability of $-\dot{h} p+h \dot{p}$ (by Lemmas A.4 and A.5) and integration by parts (Lemma A.6) in the above deduction. Recall that $h(\cdot, s)=|\nabla u|^{2}(\cdot, s)$, which implies that $-\dot{h}+\Delta h=2|\nabla \nabla u|^{2}$. Plugging this into the above equation and then integrating both sides of the equation over time, we obtain (A.12).

Lemma A.8. Same conditions as that in Lemma A.4. Fix $T>0, y \in X$, then we have

$$
\lim _{b \rightarrow T^{-}} \int_{X} h(x, T-b) p(b, x, y) d \mu_{x}=\int_{X}|\nabla f|^{2} p(T, x, y) d \mu_{x} .
$$


Proof. Choose an open set $\Omega$ such that supp $f \Subset \Omega \Subset \mathcal{R}$. Note that $u$ is a smooth heat solution on $\Omega \times[0, \infty)$. Then it is clear that

$$
\begin{aligned}
\lim _{b \rightarrow T^{-}} \int_{\Omega} h(x, T-b) p(b, x, y) d \mu_{x} & =\int_{\Omega}|\nabla f|^{2} p(T, x, y) d \mu_{x} \\
& =\int_{X}|\nabla f|^{2} p(T, x, y) d \mu_{x} .
\end{aligned}
$$

Therefore, in order to show (A.13), it suffices to show that

$$
\lim _{b \rightarrow T^{-}} \int_{X \backslash \Omega} h(x, T-b) p(b, x, y) d \mu_{x}=0 .
$$

However, by the uniform bound of $p(b, \cdot, \cdot)$ when $b \rightarrow T$, this equation can be deduced from

$$
\lim _{b \rightarrow T^{-}} \int_{X \backslash \Omega} h(x, T-b) d \mu_{x}=0 .
$$

Recalling that

$$
\begin{aligned}
& u(x, s)=\int_{X} f(z) p(s, z, x) d \mu_{z}, \\
& h(x, s)=|\nabla u|(x, s) \leqslant \int_{\operatorname{supp} f}|f|(z)\left|\nabla_{x} p\right|(s, z, x) d \mu_{z} .
\end{aligned}
$$

By the exponential decay of $p$ and $\Delta p$, for every $w \in X, z \in \operatorname{supp} f$, it is not hard to see that

$$
\begin{aligned}
\int_{B(w, 1)}\left|\nabla_{x} p\right|(s, z, x) d \mu_{x} & \leqslant C\left(\int_{B(w, 1)}\left|\nabla_{x} p\right|^{2}(s, z, x) d \mu_{x}\right)^{1 / 2} \\
& \leqslant C_{1} s^{-n-1} e^{\left(-d^{2}\left(w, z_{0}\right)+D^{2}\right) / C_{2} s}
\end{aligned}
$$

where $z_{0}$ is a fixed point in supp $f, D$ is the diameter of $\Omega, 0<s<1$. It follows that

$$
h(x, s)=|\nabla u|(x, s) \leqslant C s^{-n-1} e^{-\left(d^{2}\left(x, z_{0}\right)+D^{2}\right) / C_{2} s} .
$$

Suppose $x \in X \backslash \Omega$, then $d\left(x, z_{0}\right) \geqslant c_{0}>0$ always. Then (A.14) follows from (A.15), the Euclidean volume growth estimate and direct calculation.

Now we can finish the proof of Proposition 2.22.

Proof of Proposition 2.22. It suffices to check the three steps mentioned in the strategy. 
We first check that $\dot{\Psi}_{0}(s)=2 \Psi(s)$ for each $s \in(0,1)$. Formal calculation shows that

$$
\begin{aligned}
\dot{\Psi}_{0}(s)= & \int_{X}\left(\frac{d}{d s} u^{2}(1-s, x)\right) p(s, x, y) d \mu_{x}+\int_{X} u^{2}(1-s, x) \Delta p(s, x, y) d \mu_{x} \\
= & \int_{X}\{-2 u(x, 1-s) \dot{u}(x, 1-s)\} p(s, x, y) d \mu_{x} \\
& +\int_{X} u^{2}(1-s, x) \Delta p(s, x, y) d \mu_{x} .
\end{aligned}
$$

By the boundedness of $u, \dot{u}$ and exponential decay of $p$ and $\Delta p$, the above formal calculation is in fact rigorous for each $s \in(0,1)$. Moreover, $u^{2}(1-s, \cdot) \in L^{\infty}(X) \cap$ $N^{1,2}(X)$. Since $\Delta p$ has exponential decay, similar to Lemma A.6, one can have integration by parts to obtain

$$
\int_{X} u^{2}(1-s, x) \Delta p(s, x, y) d \mu_{x}=\int_{X} p(s, x, y) \Delta u^{2}(1-s, x) d \mu_{x} .
$$

Therefore, we have

$$
\begin{aligned}
\dot{\Psi}_{0}(s)= & \int_{X}\{-2 u(x, 1-s) \dot{u}(x, 1-s)\} p(s, x, y) d \mu_{x} \\
& +\int_{X}\left\{2 u(x, 1-s) \Delta u(x, 1-s)+2|\nabla u|^{2}(x, 1-s)\right\} p(s, x, y) d \mu_{x} \\
= & 2 \int_{X}|\nabla u|^{2}(x, 1-s) p(s, x, y) d \mu_{x}=2 \Psi(s) .
\end{aligned}
$$

So we checked the first step. However, the second step follows from Lemma A.7, the third step follows from Lemma A.8. Therefore, the proof of Proposition 2.22 is complete.

\section{Appendix B. Perturbation technique}

We often meet the problem of decomposing a manifold $M$ by regularity scales, for example cvr. Although such regularity scale functions are not smooth in general, they satisfy local Harnack inequalities (see inequality (3.18)). In this section, we show that there is a general way to perturb the regularity scale functions to smooth functions, while keeping the major properties of regularity scales. The perturbation method is a standard application of the proof of partition of unity. 
Proposition B.1 (Perturbation of general functions with local Harnack inequality). Suppose $K$ is a big positive constant, $f$ is a map from Riemannian manifold $M^{2 n}$ to $\left(0, K^{-1}\right]$ with the following local Harnack inequality

$$
K^{-1} f(x)<f(y)<K f(x), \quad \forall y \in B\left(x, K^{-1} f(x)\right) .
$$

Suppose each geodesic ball of radius $r$ has volume ratio in $\left(\kappa, \kappa^{-1}\right)$ whenever $0<r<1$. Then there exist a constant $C=C(n, \kappa, K)$ and smooth function $\tilde{f}$ such that

$$
K^{-1} f<\tilde{f}<C f, \quad|\nabla \tilde{f}|<C .
$$

Furthermore, $\tilde{f}$ also satisfies the local Harnack inequality

$$
\tilde{K}^{-1} \tilde{f}(x)<\tilde{f}(y)<\tilde{K} \tilde{f}(x), \quad \forall y \in B\left(x, \tilde{K}^{-1} \tilde{f}(x)\right)
$$

for some $\tilde{K}=\tilde{K}(n, \kappa, K)$.

Proof. $M$ can be covered by $\bigcup_{x \in M} B\left(x, 0.01 K^{-1} f(x)\right)$. By Vitali covering lemma, we can find countably many points $x_{i} \in M$ such that $M \subset \bigcup_{i} B\left(x_{i}\right.$, $\left.0.1 K^{-1} f\left(x_{i}\right)\right)$ and $B\left(x_{i}, 0.01 K^{-1} f\left(x_{i}\right)\right)$ are disjoint to each other. Let $\eta_{i}$ be a smooth function supported on $B\left(x_{i}, 0.2 K^{-1} f\left(x_{i}\right)\right)$ such that $\eta_{i} \equiv 1$ on $B\left(x_{i}\right.$, $\left.0.1 K^{-1} f\left(x_{i}\right)\right)$. Moreover, $\left|\nabla \eta_{i}\right| \leqslant 100 K / f\left(x_{i}\right)$. Fix $i$, let $B\left(x_{j}, 0.2 K^{-1} f\left(x_{j}\right)\right)$ be a ball with nonempty intersection with $B\left(x_{i}, 0.2 K^{-1} f\left(x_{i}\right)\right)$. Denote all such $j$ 's by $J_{i}$. By triangle inequality, we have

$$
d\left(x_{i}, x_{j}\right)<0.2 K^{-1}\left(f\left(x_{i}\right)+f\left(x_{j}\right)\right)<K^{-1} \max \left\{f\left(x_{i}\right), f\left(x_{j}\right)\right\} .
$$

It follows from (B.1) that $K^{-1} f\left(x_{i}\right)<f\left(x_{j}\right)<K f\left(x_{i}\right)$. In particular, we have $d\left(x_{i}, x_{j}\right)<f\left(x_{i}\right)$ and consequently $B\left(x_{j}, 0.01 K^{-1} f\left(x_{j}\right)\right) \subset B\left(x_{i}, 1.01 f\left(x_{i}\right)\right)$. By the disjoint property, we obtain

$$
\sum_{j \in J_{i}}\left|B\left(x_{j}, 0.01 K^{-1} f\left(x_{j}\right)\right)\right|<\left|B\left(x_{i}, 1.01 f\left(x_{i}\right)\right)\right| .
$$

Now we apply the lower bound $f\left(x_{j}\right)>K^{-1} f\left(x_{i}\right)$ and the volume ratio's twoside bound. The above inequality implies that

$$
\left|J_{i}\right| \cdot \kappa\left(0.01 K^{-2} f\left(x_{i}\right)\right)^{2 n}<\kappa^{-1}\left(1.01 f\left(x_{i}\right)\right)^{2 n} .
$$

Therefore, $\left|J_{i}\right|<\kappa^{-2}\left(101 K^{2}\right)^{2 n}$, which we denoted by $C=C(n, \kappa, K)$. According to the definition of $\eta_{j}$, we know that any point in $M$ can at most locate in the support of $C$ number of $\eta_{j}$ 's. Now we define

$$
\tilde{f}(x) \triangleq \sum_{i} f\left(x_{i}\right) \eta_{i}(x), \quad \forall x \in M .
$$


At every point $x$, there is a neighborhood of $x$ such that the above sum is a sum of at most $C$ nonzero terms of smooth functions. Therefore, $\tilde{f}$ is smooth. Choose an arbitrary point $x \in M$ and assume $x \in B\left(x_{i}, 0.1 K^{-1} f\left(x_{i}\right)\right)$. Since $0 \leqslant \eta_{i} \leqslant 1$, recalling the definition of $J_{i}$, we have

$$
\begin{aligned}
\tilde{f}(x) & =\sum_{j \in J_{i}} f\left(x_{j}\right) \eta_{j}(x) \leqslant \sum_{j \in J_{i}} f\left(x_{j}\right) \leqslant K \sum_{j \in J_{i}} f\left(x_{i}\right) \\
& =K\left|J_{i}\right| f\left(x_{i}\right) \leqslant C K f\left(x_{i}\right)<C K^{2} f(x),
\end{aligned}
$$

where we used (B.1) and the fact $d\left(x, x_{i}\right)<0.1 K f\left(x_{i}\right)$ in the last step. Clearly, we have

$$
\tilde{f}(x) \geqslant f\left(x_{i}\right) \eta_{i}(x)=f\left(x_{i}\right)>K^{-1} f(x) .
$$

Therefore, we obtain $K^{-1} f(x)<\tilde{f}(x)<C K^{2} f(x)$. In light of the arbitrary choice of $x$, we obtain the first part of (B.2), by adjusting $C$ if necessary. The second part of (B.2) follows from the following direct calculation.

$$
|\nabla \tilde{f}(x)| \leqslant \sum_{j \in J_{i}} f\left(x_{j}\right)\left|\nabla \eta_{j}\right| \leqslant \sum_{j \in J_{i}} 100 K \leqslant 100 K\left|J_{i}\right|<C .
$$

The local Harnack inequality (B.3) of $\tilde{f}$ follows from the combination of (B.1) and the first part of (B.2), by adjusting $K$ to $\tilde{K}=C K$ for some $C=C(n, \kappa, K)$.

In our application of Proposition B.1, we typically let $f=\min \left\{K^{-1}, \operatorname{cvr}(\cdot)\right\}$. By Proposition 3.15, the function $f$ satisfies local Harnack inequality (B.1). Then Proposition B.1 guarantees the existence of a smooth function $\tilde{f}$, which is comparable to $f$ and also satisfies local Harnack inequality, with bounded gradient. Since $\tilde{f}$ has better regularity and its value is comparable to cvr, it is convenient to use the level sets of $\tilde{f}$ to decompose the underlying manifold $M$.

Corollary B.2 (Perturbation of the level sets of cvr). Suppose $\operatorname{cr}(M)>1$, $\xi_{0}=\xi_{0}(n, \kappa)$ is a very small constant. Suppose $\xi=\operatorname{cvr}(x)<\xi_{0}$ for some $x \in$ $B\left(x_{0}, 0.5\right)$. Then there is a smooth $(2 n-1)$-dimensional hypersurface $\Sigma_{\xi}$ such that:

(a) $C^{-1} \xi<\operatorname{cvr}(y)<C \xi$ for every $y \in \Sigma_{\xi}$;

(b) $\left|\Sigma_{\xi} \cap B\left(x_{0}, 1\right)\right|_{\mathcal{H}^{2 n-1}}<C \xi^{2 p_{0}-1}$.

Here $C=C(n, \kappa, K)=C(n, \kappa)$ since $K$ is the constant depending on $n, \kappa$ in Proposition 3.15. 
Proof. Let $f=\min \left\{K^{-1}, \operatorname{cvr}(\cdot)\right\}$, which satisfies (B.1) by Proposition 3.15. Therefore, Proposition B.1 can be applied. We perturb $f$ to a smooth function $\tilde{f}$ such that inequality (B.2) hold. At the given point $x$, we have $\tilde{f}(x)<C \xi<C \xi_{0}<$ $K^{-2}$ since $\xi_{0}$ is chosen very small. Recalling that $\operatorname{cr}(M)>1$, the density estimate (see Proposition 3.10) guarantees the existence of point $y \in B\left(x_{0}, 1\right)$ such that $\operatorname{cvr}(y)>K^{-1}$. Clearly, $f(y)=K^{-1}$ by definition. Therefore, $\tilde{f}(y)>K^{-2}$ by (B.2). In light of the continuity of $\tilde{f}$, we have $\tilde{f}^{-1}(a) \cap B\left(x_{0}, 1\right) \neq \emptyset$ for each $a \in\left[C \xi, K^{-2}\right]$. Applying coarea formula, we obtain

$$
\begin{aligned}
& \int_{C \xi}^{2 C \xi}\left|\tilde{f}^{-1}(a) \cap B\left(x_{0}, 1\right)\right|_{\mathcal{H}^{2 n-1}} d t \\
& \quad \leqslant \int_{\tilde{f}^{-1}([C \xi, 2 C \xi]) \cap B\left(x_{0}, 1\right)}|\nabla \tilde{f}| d \mu \leqslant C \int_{\tilde{f}^{-1}([0,2 C \xi]) \cap B\left(x_{0}, 1\right)} 1 d \mu,
\end{aligned}
$$

where we applied (B.2) in the last step. Note that $\tilde{f}$ is comparable to cvr on small values, our conditions provide $\operatorname{cr}(M)>1$, the last term in the above inequality can be bounded by the density estimate (see inequality (3.13)). Therefore, we have

$$
\int_{C \xi}^{2 C \xi}\left|\tilde{f}^{-1}(a) \cap B\left(x_{0}, 1\right)\right|_{\mathcal{H}^{2 n-1}} d t<C \xi^{2 p_{0}} .
$$

By Sard theorem and mean-value inequality, we can choose $a_{0} \in[C \xi, 2 C \xi]$ to be a regular value of $\tilde{f}$ and it satisfies

$$
\left|\tilde{f}^{-1}\left(a_{0}\right)\right|_{\mathcal{H}^{2 n-1}} \leqslant \frac{2}{C \xi} \int_{C \xi}^{2 C \xi}\left|\tilde{f}^{-1}(a)\right|_{\mathcal{H}^{2 n-1}} d t \leqslant C \xi^{2 p_{0}-1} .
$$

Let $\Sigma_{\xi}$ be $\tilde{f}^{-1}\left(a_{0}\right)$. Then it satisfies all the requirements.

Because of the properties (a) and (b) of Corollary B.2, we can regard $\Sigma_{\xi}$ as a perturbation of $\partial \mathcal{F}_{\xi}$ in many applications.

COROllary B.3 (Perturbation of distance function). Let

$$
X=\mathcal{R} \cup \mathcal{S} \in \widetilde{\mathscr{K} \mathscr{S}}(n, \kappa) .
$$

Let $f=\min \{0.1, d(\cdot, \mathcal{S})\}$. Then there is a smooth function $\tilde{f}$ such that

$$
0.1 f<\tilde{f}<C f, \quad|\nabla \tilde{f}|<C .
$$

Furthermore, for each small positive number $\xi$, large positive number $H$ and point $x_{0} \in X$ satisfying $B\left(x_{0}, H\right) \cap \mathcal{S} \neq \emptyset$, we can find a smooth $(2 n-1)$ dimensional hypersurface $\Sigma_{\xi} \subset \mathcal{R}$ such that: 
(a) $C^{-1} \xi<d(y, \mathcal{S})<C \xi$ for every $y \in \Sigma_{\xi}$;

(b) $\left|\Sigma_{\xi} \cap B\left(x_{0}, H\right)\right|_{\mathcal{H}^{2 n-1}}<L \xi^{2}$.

All the $C$ in this corollary depend only on $n$ and $\kappa$, the constant $L$ depends on $n, \kappa$ and the ball $B\left(x_{0}, H\right)$.

Proof. The proof of (B.4) follows from the proof of inequality (B.2) by letting $M=\mathcal{R}$ and $K=10$, with the following facts in mind. First, it is clear that $f$ satisfies the local Harnack inequality (B.1) by triangle inequality. Second, for each $x \in \mathcal{R}$, we have

$$
B\left(x, 0.2 K^{-1} f(x)\right) \subset B(x, 0.02 d(x, \mathcal{S})) \subset \mathcal{R} .
$$

Therefore, it makes sense to construct smooth cutoff functions supported on $B(x$, $\left.0.2 K^{-1} f(x)\right)$.

The existence of such $\Sigma_{\xi}$ follows from the proof of Corollary B.2 and the Minkowski codimension assumption of $\mathcal{S}$. In other words, we have

$$
\left|\left\{x \in B\left(x_{0}, H\right) \mid d(x, \mathcal{S})<\xi\right\}\right|<L \xi^{3} .
$$

Similar to the proof of Corollary B.2, the property of $\Sigma_{\xi}$ is the application of Sard theorem and coarea formula.

\section{References}

[1] U. Abresch and D. Gromoll, 'On complete manifolds with nonnegative Ricci curvature', J. Amer. Math. Soc. 3(2) (1990), 355-374.

[2] M. Anderson, 'Convergence and rigidity of manifolds under Ricci curvature bounds', Invent. Math. 102 (1990), 429-445.

[3] D. Bakry, 'On Sobolev and logarithmic inequalities for Markov semigroups', in New Trends in Stochastic Analysis (Charingworth, 1994) (World Scientific Publishing, River Edge, NJ, 1997), 43-75.

[4] D. Bakry and M. Emery, 'Diffusions hypercontractives', Seminaire de probabilities XIX (1983/84), 177-206.

[5] J. Cheeger, 'Differentiability of Lipschitz functions on metric measure spaces', Geom. Funct. Anal. 9 (1999), 428-517.

[6] J. Cheeger, Degeneration of Riemannian Metrics Under Ricci Curvature Bounds, Publications of the Scuola Normale Superiore, Edizioni della Normale, October 1, 2001.

[7] J. Cheeger, 'Integral Bounds on curvature, elliptic estimates and rectifiability of singular sets', Geom. Funct. Anal. 13 (2003), 20-72.

[8] J. Cheeger and T. H. Colding, 'Lower bounds on Ricci curvature and the almost rigidity of warped products', Ann. of Math. (2) 144(1) (1996), 189-237.

[9] J. Cheeger and T. H. Colding, 'On the structure of spaces with Ricci curvature bounded below. I', J. Differential Geom. 45 (1997), 406-480. 
[10] J. Cheeger, T. H. Colding and G. Tian, 'On the singularities of spaces with bounded Ricci curvature', Geom. Funct. Anal. 12 (2002), 873-914.

[11] J. Cheeger and A. Naber, 'Lower bounds on Ricci curvature and quantitative behavior of singular sets', Invent. Math. 191(2) (2013), 321-339.

[12] J. Cheeger and J. Simons, 'Differential characters and geometric invariants', in Geometry and Topology (College Park, MD, 1983/84), Lecture Notes in Mathematics, 1167 (Springer, 1985), 50-80.

[13] T. H. Colding, 'Ricci curvature and volume convergence', Ann. of Math. (2) 145 (1997), 477-501.

[14] T. H. Colding and A. Naber, 'Sharp Hölder continuity of tangent cones for spaces with a lower Ricci curvature bound and applications', Ann. of Math. (2) 176(2) (2012), 1173-1229.

[15] X. X. Chen and B. Wang, 'Space of Ricci flows (I)', Comm. Pure Appl. Math. 65(10) (2012), 1399-1457.

[16] X. X. Chen and B. Wang, 'Space of Ricci flows (II)', Preprint, 2014, arXiv:1405.6797.

[17] X. X. Chen and B. Wang, 'Space of Ricci flows (II)_Part B: weak compactness of the flows', Preprint.

[18] X. X. Chen and B. Wang, 'Further details of "Space of Ricci flows (II)", Preprint, available at www.math.wisc.edu/ bwang/HTfurtherdetails.pdf.

[19] S. Y. Cheng and S. T. Yau, 'Differential equations on Riemannian manifolds and their geometric applications', Comm. Pure Appl. Math. 28(3) (1975), 333-354.

[20] T. Coulhon and L. Saloff-Coste, 'Isopérimétrie pour les groupes et les variétés', Rev. Mat. Iberoam. 9(2) (1993), 293-314.

[21] C. B. Croke, 'Some isoperimetric inequalities and eigenvalue estimates', Ann. Sci. Éc. Norm. Supér. 13 (1980), 419-435.

[22] L. C. Evans, Partial Differential Equations, 2nd edn, Graduate Studies in Mathematics, 19 (American Mathematical Society, 2010).

[23] K. Falconer, Fractal Geometry, Mathematical Foundations and Applications (John Wiley and Sons, 1990).

[24] M. Fukushima, Y. Oshima and M. Takeda, Dirichlet Forms and Symmetric Markov Processes (de Gruyter, Berlin, 1994).

[25] N. Gigli, 'The splitting theorem in non-smooth context', Preprint, 2013, arXiv:1302.5555.

[26] B. Greene, The Elegant Universe (W.W. Norton and Co., 2003), ISBN 0-393-05858-1.

[27] A. A. Grigor'yan, 'The heat equation on noncompact Riemannian manifolds (Russian)', Mat. Sb. 182(1) (1991), 55-87. English translation: Math. USSR, Sb. 72(1) (1992), 47-77.

[28] R. S. Hamilton, 'A matrix Harnack estimate for the heat equation', Comm. Anal. Geom. 1(1) (1993), 113-126.

[29] R. S. Hamilton, 'A compactness property for solutions of the Ricci flow', Amer. J. Math. 117(3) (1995), 545-572.

[30] J. Kinnunen and N. Shanmugalingam, 'Regularity of quasi-minimizers on metric spaces', Manuscripta Math. 105 (2001), 401-423.

[31] P. Koskela, K. Rajala and N. Shanmugalingam, 'Lipschitz continuity of Cheeger-harmonic functions in metric measure spaces', J. Funct. Anal. 202 (2003), 147-173.

[32] P. Koskela and Y. Zhou, 'Geometry and analysis of Dirichlet forms', Adv. Math. 231(5) (2012), 2755-2801.

[33] P. Li and S. T. Yau, 'On the parabolic kernel of the Schrödinger operator', Acta Math. 156 (1986), 153-201.

[34] G. Perelman, 'The entropy formula for the Ricci flow and its geometric applications', Preprint, arXiv:math.DG/0211159. 
[35] N. Shanmugalingam, 'Newtonian spaces: an extension of Sobolev spaces to metric measure spaces', Rev. Mat. Iberoam. 16(2) (2000), 243-279.

[36] N. Shanmugalingam, 'Harmonic functions on metric spaces', Illinois J. Math. 45(3) (2001), 1021-1050.

[37] L. Saloff-Coste, 'Uniformly elliptic operators on Riemannian manifolds', J. Differential Geom. 36 (1992), 417-450.

[38] L. Saloff-Coste, 'A note on Poincaré, Sobolev, and Harnack inequalities', Int. Math. Res. Not. IMRN 2 (1992), 27-38.

[39] L. Saloff-Coste, 'Sobolev inequalities in familiar and unfamiliar settings', in Sobolev Spaces in Mathematics I, Int. Math. Ser., 8 (Springer, New York, 2009), 299-343.

[40] K. T. Sturm, 'Analysis on local Dirichlet spaces. I. Recurrence, conservativeness and $L^{p}$ Liouville properties', J. Reine Angew. Math. 456 (1994), 173-196.

[41] K. T. Sturm, 'Analysis on local Dirichlet spaces. II. Upper Gaussian estimates for the fundamental solutions of parabolic equations', Osaka J. Math. 32(2) (1995), 275-312.

[42] K. T. Sturm, 'Analysis on local Dirichlet spaces. III. The parabolic Harnack inequality', J. Math. Pures Appl. 75(9) (1996), 273-297.

[43] G. F. Wei, 'Manifolds with a lower Ricci curvature bound', Preprint, arXiv:math/0612107.

[44] S. H. Zhu, 'The comparison geometry of Ricci curvature', in Comparison Geometry 30 (MSRI Publications, 1997), 221-262. 